\title{
The Metallomimetic Chemistry of Boron
}

Marc-André Légaré, ${ }^{a, b, \star}$ Conor Pranckevicius, ${ }^{a, b, *}$ Holger Braunschweiga,

a Institute for Inorganic Chemistry, Julius-Maximilians-Universität Würzburg, Am Hubland, 97074

Würzburg (Germany). ${ }^{\mathrm{b}}$ Institute for Sustainable Chemistry \& Catalysis with Boron, Julius-MaximiliansUniversität Würzburg, Am Hubland, 97074 Würzburg (Germany).

* These authors contributed equally

Abstract: The study of main-group molecules that behave and react similarly to transition metal (TM) complexes has attracted significant interest in the recent decades. Most notably, the attractive idea of eliminating the all-too-often rare and costly metals from catalysis has motivated efforts to develop main-group-element-mediated reactions. Main-group elements, however, lack the electronic flexibility of many TM complexes that arise from combinations of empty and filled $d$-orbitals and that seem ideally suited to bind and activate many substrates. In this Review, we look at boron, an element which, despite its non-metal nature, low atomic weight, and relative redox staticity has achieved great milestones in terms of TM-like reactivity. We show how in inter-element cooperative systems, diboron molecules and hypovalent complexes, the fifth element can acquire a truly metallomimetic character. As we discuss, this character is particularly strikingly demonstrated by the reactivity of boron-based molecules with $\mathrm{H}_{2}, \mathrm{CO}$, alkynes, alkenes and even with $\mathrm{N}_{2}$.

\subsection{Introduction}

The transition elements are defined by their $d$-orbital sub-shell which has the unique situation of being partially filled. ${ }^{1}$ These $d$-orbitals, which are part of the valence shell of the transition metals, have become the salient feature of their chemistry and give them a unique place in the periodic table and in the realms of scientific interests and applications. In the most challenging steps of many catalytic cycles, the interplay of electron density and electron-deficiency that is a direct consequence of the partially filled $d$-shell enables complex processes that are not trivial for other elements. ${ }^{2}$ Their large range of oxidation states, the flexibility of their coordination patterns and the reversibility of their transformations are predicated on this unique electronic arrangement. It is thus unsurprising that transition metals, in materials and in molecular complexes, are at the forefront of the catalytic sciences, which often focus on designing precisely tuned arrangements of ligands and metal atoms in order to bind small molecules, cleave bonds and couple building blocks in innumerable applications (Figure 1). ${ }^{3}$

However, the scarcity, high cost, toxicity and environmental issues associated with the extraction and use of transition metals, as well as the fundamental scientific instinct of pushing back the boundaries of knowledge, have sparked considerable interest in investigating the possibility of hitherto transition-metal-exclusive reactions using purely main-group compounds. This new field of chemistry, which we have termed 'main-group metallomimetics', ${ }^{4}$ has gained 
considerable traction in recent years and has led to the discovery of both conceptual reactions and applicable catalytic reactions.

The design and preparation of main-group metallomimetics, however, is not trivial and relies on the application of unusual strategies and the development of new concepts. At first glance, the usual properties of main group elements would disqualify them from reacting in analogous ways to transition metals: where many transition metals can access a range of oxidation states and often can relatively freely transition from one to the other, main-group elements usually display a strong affinity for one or two oxidation states and remain relatively redox-static. Furthermore, in order to achieve their preferred oxidation state and to follow the empirical octet rule, main-group elements tend to form complexes of invariable coordination numbers and of set geometries that can be predicted using the VSEPR model. For main-group compounds, these geometries and coordination numbers are often associated with clear nucleophilic or electrophilic properties, which are in contrast with the electronic interplay possible with TM complexes.

The metallomimetic chemistry of the heavier main group elements has recently been reviewed by Power. ${ }^{5}$ However, the natural abundance and low toxicity of light main-group elements of the second period make them especially attractive candidates for the design of metallomimetics. Among these elements, boron is being increasingly found in atypical oxidation states and molecular arrangements and deserves to be described independently on account of its unique chemistry.

\subsection{Definition of Metallomimetic Compounds and Scope of this Review}

The reactivity of boron and of the other light main-group elements is limited by their rigid electronic properties. For this reason, it is not common for complexes of boron to react with substrates that interact preferably with a combination of filled and empty orbitals. Among the reactions that are atypical for main-group compounds are: a) the fixation of small molecules that bind to metal centers through $\sigma$-donation and m-backbonding (e.g. $\mathrm{CO}, \mathrm{N}_{2}, \eta^{2}$-alkenes and alkynes), and b) the cleavage of unactivated bonds (e.g. $\mathrm{C}-\mathrm{H}$ and $\mathrm{H}_{2}$ activation) (Figure 2).

Boron-containing systems that are capable of these reactions will be the focus of this review. The unique strategies and concepts that enable boron to act in a manner reminiscent of the reactivity of transition-metal catalysts is the core of the understanding of boron metallomimetics. These approaches can be divided into three categories (Figure 2): in the first, the boron center effects its metallomimetic reactivity through the cooperative action of another non-metal element. This approach includes ambiphilic systems and the frustrated Lewis pair (FLP) $)^{6,7}$ paradigm that has been formulated in the previous decade around boron-based catalysts.

The second class of metallomimetics is defined by the cooperative reactivity of more than one boron center. This type of metallomimetic includes the unique reactivity of B-B single and multiple bonds within diborane, diborenes, diboracumulenes and diborynes, as well as that of unusual species that feature proximal boron centers. In many of these cases, one boron center provides an empty orbital while either the B-B bond or another nucleophilic boron atom provides the possibility of backdonation. 
Finally, we will also outline the chemistry of metallomimetics that feature the reactivity of a single boron atom as the active site; most often in an atypical oxidation state. In the most striking of these cases, the complexes affect reactivity through the cooperation of empty and filled boron-centered orbitals. While this class of reactive compounds is still emerging, recent advances in the field of borylene chemistry have clearly demonstrated the ability of mononuclear boron complexes to behave like transition metals.

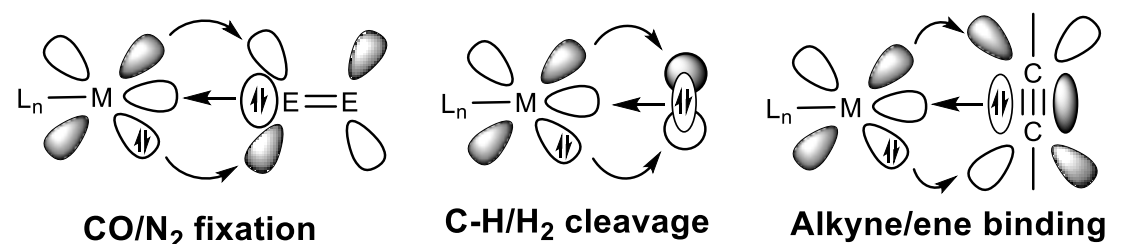

Figure 1. The Dewar-Chatt-Duncanson model of TM-based substrate activation

\begin{tabular}{|c|c|c|c|}
\hline Metallomimetic & Empty Orbital & Filled Orbital & Orbital Depiction \\
\hline $\begin{array}{l}\text { FLP-ambiphilic } \\
\text { (Chapter 1) }\end{array}$ & Boron & Lewis Base & \\
\hline $\begin{array}{l}\text { Dinuclear Boron } \\
\quad(\text { Chapter 2) }\end{array}$ & Boron & $\begin{array}{c}\text { Boron / } \\
\text { B-B bond }\end{array}$ & \\
\hline $\begin{array}{l}\text { Single-site Boron } \\
\quad \text { (Chapter 3) }\end{array}$ & Boron & Boron & \\
\hline
\end{tabular}

Figure 2. Description of boron-based metallomimetics that are the topic of this Review.

\section{Metallomimetic boron - FLPs}

\subsection{Introduction - FLP Activation}

The partially filled d-subshell ${ }^{1}$ of the transition metals (TMs) allows them to interact with small molecules according to the Dewar-Chatt-Duncanson model. ${ }^{8,9}$ In a single-site TM species, a combination of vacant and filled d-orbitals of appropriate symmetry activate a substrate through interaction with its frontier molecular orbitals. These orbital interactions weaken bonds within a substrate and facilitate reaction steps such as migration, insertion, as well as redoxactive processes such as oxidative addition and reductive elimination. In contrast, tricoordinate boron(III) compounds have only a single vacant p-orbital and no non-bonding electrons. However, in combination with a second element possessing a filled non-bonding orbital, reactivity that mimics transition metal centers can be achieved.

This approach underpins the chemistry of frustrated Lewis pairs (FLPs), a concept that was introduced by Stephan and coworkers in $2007 .{ }^{10}$ FLPs are undoubtedly the most mature field of boron metallomimetics; having emerged as a powerful tools for the activation of $\mathrm{H}_{2}, \mathrm{CO}_{2}$, 
$\mathrm{CO}, \mathrm{N}_{2} \mathrm{O}, \mathrm{NO}, \mathrm{SO}_{2}, \mathrm{Si}-\mathrm{H}$, olefins, and alkynes. Since the chemistry of FLPs has been extensively reviewed in recent literature,,$^{6,711-16}$ it will be the scope of this section to explore mechanistic themes of boron(III)-based FLP transformations with small molecules including $\mathrm{H}_{2}$, olefins, alkynes, $\mathrm{CO}$, and arene $\mathrm{C}-\mathrm{H}$ bonds, and examine their relationship to analogous $\mathrm{TM}$ based transformations.

Within the FLP paradigm, one molecular component functions as the Lewis acid (LA), and the other as the Lewis base (LB), which through steric encumberment or reduced electronic overlap are prevented from strongly interacting with one another. Consequently, the reactivity of both the Lewis acid (usually a borane) and the Lewis base (typically based on phosphorus, nitrogen, carbon, or oxygen) is preserved in the reaction medium and can be simultaneously applied to the substrate. ${ }^{17,18}$ Electron transfer $(E T)$ is therefore achieved between the substrate and via a vacant $p$-orbital on boron, in combination with a filled orbital on the organic base.

\subsection{Dihydrogen}

While early examples of 'FLP-type' reactivity utilizing bulky Lewis acids/bases had been reported in prior literature, ${ }^{19-21}$ it was the seminal discovery of metallomimetic reversible $\mathrm{H}_{2}$ cleavage by Stephan and coworkers by an intramolecular perfluorophenylene-linked phosphinoborane that proved to be the ignition point of the field. ${ }^{22}$ The reactivity with $\mathrm{H}_{2}$ was quickly extended to bimolecular systems. ${ }^{23}$ The common avenues for $\mathrm{H}_{2}$ activation in both FLP systems and TM systems are presented below (Figure 3). In the case of unimolecular TM systems, it is the combination of both vacant and filled d-orbitals of appropriate symmetry that simultaneously withdraw electron density from a substrate $\mathrm{HOMO}$; in this case the filled $\sigma$-orbital of $\mathrm{H}_{2}$, and donate electron density to the corresponding LUMO; the $\sigma^{*}-\mathrm{H}_{2}$ orbital. ${ }^{24}$ Another common mechanism of substrate activation is bifunctional activation between a TM and a ligand-centered base; ${ }^{25,26}$ particularly relevant in the context of hydrogenation of polar substrates. ${ }^{27,28}$ Interestingly, even after a decade of computational examination, the exact mechanism of $\mathrm{H}_{2}$ splitting via the prototypical $\mathrm{B}\left(\mathrm{C}_{6} \mathrm{~F}_{5}\right)_{3} / \mathrm{PtBu}_{3}$ FLP is still a matter of some contention. ${ }^{29}$ However, there is general agreement on the initial formation of an FLP 'encounter complex'; ${ }^{\prime, 30-32}$ a pre-association of the Lewis acid and base substituents, which is primarily supported by secondary bonding interactions. From this point, the proposed mechanisms differ. According to the electric field (EF) picture, ${ }^{30,33,34}$ rate-limiting insertion of the $\mathrm{H}_{2}$ unit into the 'reactive pocket' of the encounter complex is followed by polarization of the $\mathrm{H}_{2}$ moiety by the electric field therein, whereupon facile scission of the $\mathrm{H}_{2}$ unit occurs with formation of the phosphonium borohydride salt. No significant orbital interactions are present in the transition state. According to the ET picture, ${ }^{17,18,35}$ the electric field induced within the encounter complex is insufficient to split the $\mathrm{H}_{2}$ moiety, and activation is supported via orbital interactions $\mathrm{sp}^{\mathrm{x}}(\mathrm{P}) \rightarrow \sigma^{*}\left(\mathrm{H}_{2}\right)$ and $\sigma\left(\mathrm{H}_{2}\right) \rightarrow \mathrm{p}(\mathrm{B})$. The transition state features significant orbital interactions of the FLP with the $\mathrm{H}_{2}$ moiety. From this point, the mechanism of delivery of $\mathrm{H}_{2}$ to the FLP partners has been described as concerted but asynchronous, ${ }^{35}$ where the $\mathrm{B}-\mathrm{H}$ bond is formed somewhat before the $\mathrm{P}-\mathrm{H}$ bond. Interestingly, while steric frustration is a primary tool to effect FLP hydrogen activation in Lewis acid/base pairs, the energetic mismatch of molecular filled/vacant orbitals within intramolecular systems can also be used to a similar end. For example, the phosphinoborane $\left(\mathrm{C}_{6} \mathrm{~F}_{5}\right)_{2} \mathrm{~B}=\mathrm{PtBu}$, which features a high lying $\mathrm{B}-\mathrm{P} \pi$-orbital and low lying $\mathrm{B}-\mathrm{P}$ 
$\Pi^{*}$-orbital, readily reacts with $\mathrm{H}_{2}$ to afford the corresponding phosphionium borate zwitterion. ${ }^{36,37}$ Exceptionally, Yamashita and Nozaki have also reported the scission of $\mathrm{H}_{2}$ by a boryllithium species, where a nucleophilic boron center functions as the Lewis base in heterolytic $\mathrm{H}_{2}$ splitting to form a borohydride and lithium hydride. ${ }^{38}$

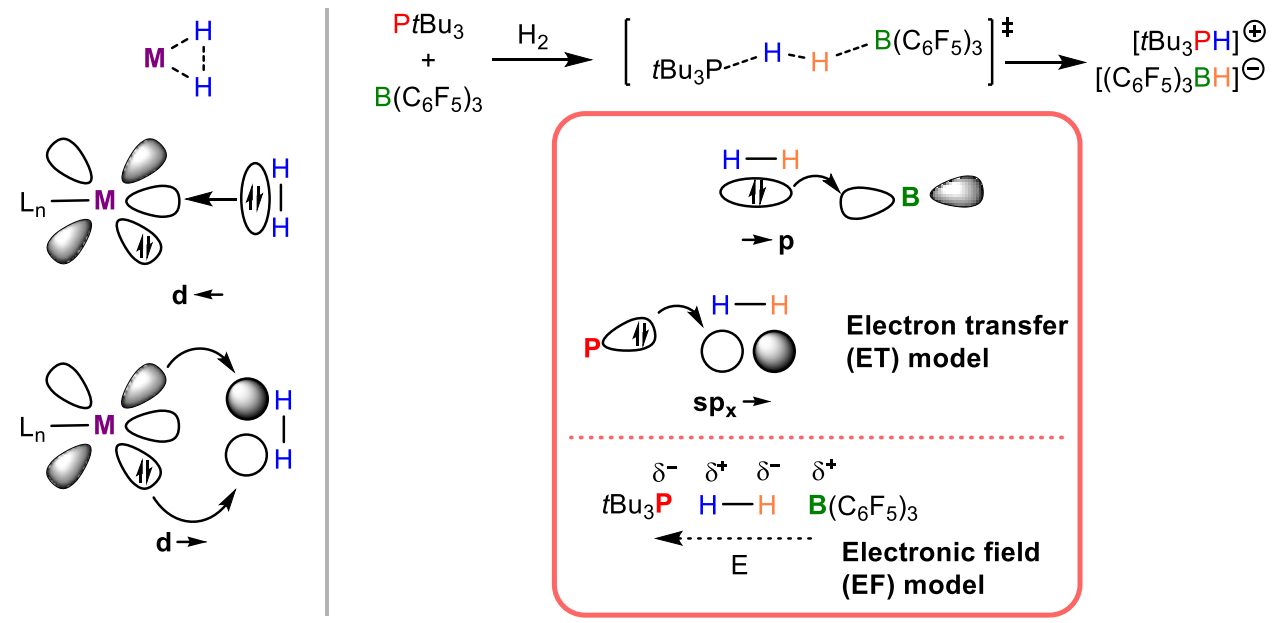

Figure 3. Interactions relevant to the activation of $\mathrm{H}_{2}$ using both TMs (left) and the $\mathrm{B}\left(\mathrm{C}_{6} \mathrm{~F}_{5}\right)_{3} / \mathrm{PtBu} \mathrm{Bu}_{3} \mathrm{FLP}$ (right).

While originally a fundamental curiosity, the metallomimetic behavior of FLPs was cemented by their ability to catalytically deliver $\mathrm{H}_{2}$ to unsaturated polarized substrates, such as imines, enamines, and silyl enol ethers, ${ }^{39-46}$ and later to more challenging substrates such as ketones, ${ }^{47-49}$ and anilines/ $\mathrm{N}$-heterocycles, ${ }^{50-55}$ and has recently been comprehensively reviewed. ${ }^{56}$ Such hydrogenations proceed via an ionic mechanism, ${ }^{39,57}$ where proton and hydride delivery to a substrate (which may also function as the FLP base if it is sufficiently nucleophilic) occurs in a stepwise manner (Figure 4, left). Tuning the acidity/hydricity of the captured $\mathrm{H}_{2}$ was paramount in achieving efficient delivery of $\mathrm{H}_{2}$, as while strongly Lewis acidic and basic FLPs cleave $\mathrm{H}_{2}$ most effectively, it is FLPs of reduced Lewis acidity/basicity that are the most suitable to deliver a reactive proton or hydride to an unsaturated substrate ${ }^{32,58-60}$ In a systematic study of imine hydrogenation catalysis by NHC-borenium cations, ${ }^{61}$ it was found that a non-bulky and electron-poor NHC supporting ligand yielded the fastest FLP hydrogenation catalyst, despite being significantly less Lewis acidic than $\mathrm{B}\left(\mathrm{C}_{6} \mathrm{~F}_{5}\right)_{3}$. The activation of dihydrogen by FLP systems is highly reminiscent of outer-sphere Noyori-type $\mathrm{H}_{2}$ activation between a Lewis acidic metal centre and a ligand-centered base, ${ }^{62,63}$ wherein similar unquenched acidity/basicity of the system affords greatly accelerated heterolytic cleavage of $\mathrm{H}_{2}$, which is subsequently delivered to unsaturated polar substrates in a concerted manner. Despite the stepwise delivery of proton and hydride in FLP systems, some success in enantioselective imine hydrogenation has been achieved via the use of chiral boranes in the activation of $\mathrm{H}_{2}{ }^{64-71}$ However, this effort is complicated by the ability of some highly Lewis acidic boranes to epimerize chiral amines. ${ }^{72}$ 

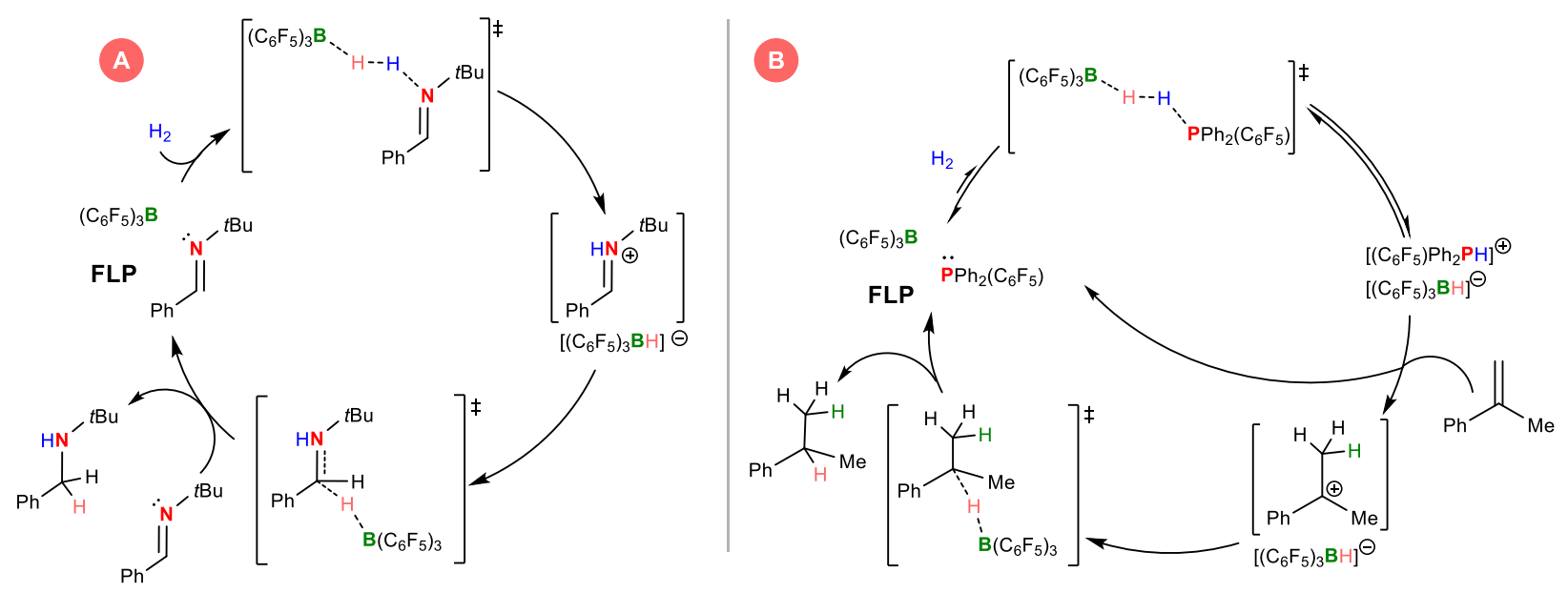

Figure 4. FLP-based catalytic hydrogenation of imines (left) and alkenes (right).

The FLP hydrogenation of non-polar functionalities, such as olefins and polycyclic aromatic species, was realized by the use of very weak and bulky bases, such as diarylamines, electrondeficient phosphines, and ethers (Figure 4, right) in combination with $\mathrm{B}\left(\mathrm{C}_{6} \mathrm{~F}_{5}\right)_{3},{ }^{73-77}$ which operates via a similar ionic mechanism described above. ${ }^{78}$ While the $\mathrm{H}_{2}$ cleavage step with such systems is disfavored and can only be observed spectroscopically at $-80^{\circ} \mathrm{C}$, the conjugate phosphonium salt formed upon reaction of the FLP system with hydrogen is now significantly acidic to protonate certain olefins to form a transient carbocation. As such, a requirement for catalytic turnover is that the carbocation formed is sufficiently long-lived that it may irreversibly react with the borohydride. Stabilization of the intermediate carbocation was typically achieved via hyperconjugation or delocalization with a neighboring $\pi$-system, therefore limiting the substrate scope to mostly conjugated and 1,1-geminally substituted olefins. Outer-sphere delivery of $\mathrm{H}_{2}$ has been proposed in the TM-catalyzed hydrogenation of the $\mathrm{C}=\mathrm{C}$ bonds of quinolines, ${ }^{79}$ however, to our knowledge has not been invoked for simple olefins.

Subsequently, Repo and coworkers ${ }^{80}$ designed a linked amine-hydroborane FLP (1) for the selective cis-hydrogenation of internal alkynes, utilizing a hydroboration $-\mathrm{H}_{2}$ cleavage protodeboronation mechanism (Figure 5), which bears a clear analogy to the classic innersphere TM hydrogenation mechanism..$^{81,82}$ This catalyst displays a high degree of chemoselectivity towards internal alkynes, as upon hydroboration with an olefin, catalyst degradation via loss of $\mathrm{C}_{6} \mathrm{~F}_{5} \mathrm{H}$ was more favorable than alkane release. A similar reaction has also been realized via a hydridoborane/silica-supported phosphine FLP. ${ }^{83}$ Additionally, Wang and coworkers also have reported that the simple, highly Lewis acidic hydroborane $\mathrm{HB}\left(\mathrm{C}_{6} \mathrm{~F}_{5}\right)_{2}$ can hydrogenate simple alkenes via a related hydroboration - hydrogenolysis mechanism, ${ }^{84}$ which will be discussed in more detail in section 4.1. 


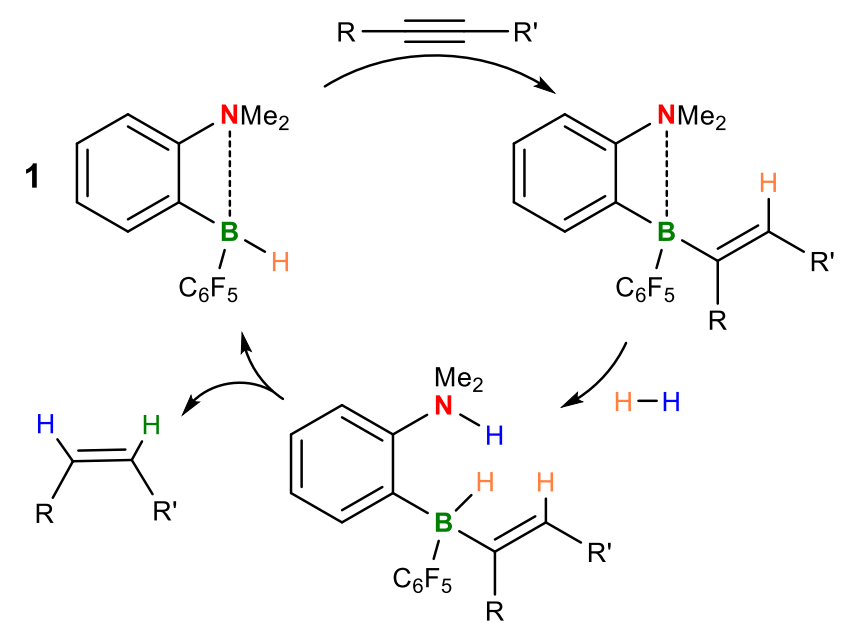

Figure 5. Hydrogenation of internal alkenes catalyzed by an amino-borane FLP.

Overall, the wealth of FLP reactivity with $\mathrm{H}_{2}$ has uncovered two main hydrogenation mechanisms. Polar hydrogenations generally take place via a stepwise 'outer-sphere' (also termed 'ionic') mechanism, which generally feature nucleophilic hydride delivery to an unsaturated substrate. In contrast, non-polar hydrogenations can operate via an 'inner-sphere' mechanism, which involves insertion of an unsaturated substrate into a borohydride (hydroboration), followed by protodeborylation or hydrogenolysis (vide infra). Such transformations directly mirror the hydrogenation mechanisms that operate with TM systems.

\subsection{Olefins}

Olefins react stoichiometrically with P/B-based FLPs to form ethylene-bridged phosphonium borate zwitterions (Figure 6, A). ${ }^{10,85}$ The transition states of such additions with phosphines have been computed to involve concerted, but asynchronous, $s^{x}(P) \rightarrow \pi^{*}$ (olefin) and $\pi($ olefin $) \rightarrow p(B)$ orbital interactions, ${ }^{86,87}$ directly analogous to the Dewar-Chatt-Duncanson model of TM olefin activation. ${ }^{8,9}$ Furthermore, such transformations bear strong mechanistic analogy to the TMcatalyzed hydroamination reaction, where an external amine attacks a TM-activated olefin to form a 2-aminoethyl ligand (outer-sphere mechanism), which can subsequently undergo further reactivity (Figure $6, \mathrm{C}){ }^{88}$ 


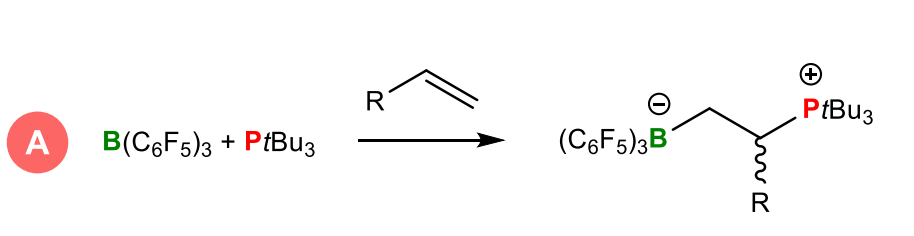

B<smiles>C=C/C=C/CC(OC(F)(F)F)(C(F)(F)F)C(F)(F)C(F)(F)C(F)(F)F</smiles>

Van der Waals complex

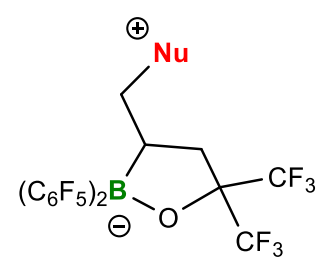

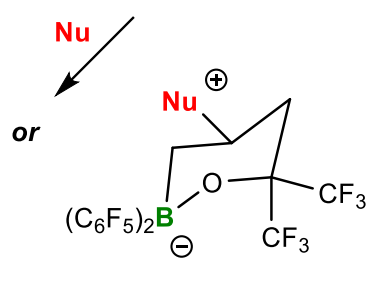

$\mathrm{Nu}=\mathrm{PR}_{3}, \mathrm{NHC}, \mathrm{py}$, amine, hydride

C

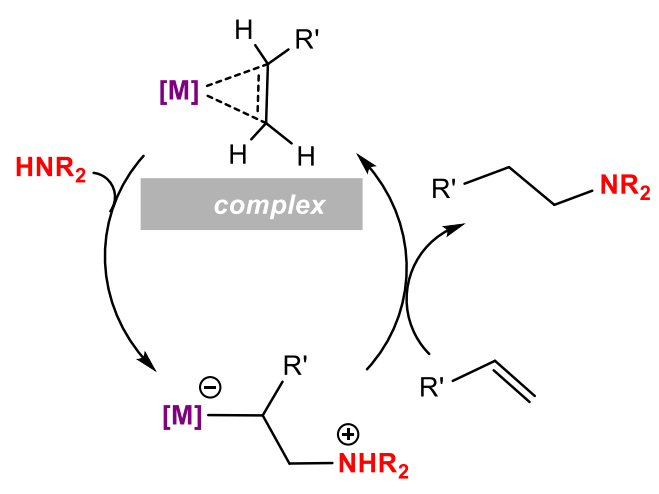

Figure 6. FLP- and TM-based activations of olefins.

Experimental evidence for the pre-interaction of olefins with the Lewis acidic unit prior to attack of the basic partner has emerged: Stephan and coworkers have prepared a strongly Lewis acidic borane bearing a pendant olefinic group (2), where NMR spectroscopic evidence of the "van der Waals complex" of the olefin pre-coordinated to the borane was presented. ${ }^{89,90}$ It has additionally been shown computationally that such van der Waals complexes are important intermediates in FLP olefin capture with simple substrates, such as ethylene. ${ }^{86}$ While such complexes are not true m-olefin complexes, and the activation they provide is very minimal, they nevertheless provide a 'pre-organization' of the reaction partners toward olefin activation that is essential for termolecular reactions. Subsequent addition of a nucleophile, such as a phosphine, amine, or N-heterocyclic carbene, to the tethered-olefin van der Waals complex results in rapid nucleophilic addition, with formation of either the five- or six-membered boracycle, depending on the steric constraints of the incoming nucleophile (Figure 6, B).

\subsection{Alkynes}


With FLPs, internal alkynes form simple 1,2-addition products much akin to their reactivity with olefins. ${ }^{91}$ However, in contrast to alkenes, which form very weak van der Waals complexes with Lewis acidic boranes, terminal alkynes have been determined computationally to form more strongly bound (but still fairly weak) $\sigma$-complexes with highly Lewis acidic boranes, ${ }^{92,93}$ which activate the alkyne by acidifying the alkynyl $\mathrm{C}-\mathrm{H}$ proton and withdrawing electron density from the $\beta$-carbon (Figure 7). Such species have an electronic structure similar to that of vinyl cations derived from electrophilic addition to alkynes. ${ }^{94}$ In the absence of an external nucleophile, reactions of terminal alkynes with strongly Lewis acidic perfluoroarylboranes typically proceed via migration of the alkynyl hydrogen to the $\beta$-carbon, concomitant with activation of a B-C bond, resulting in the formation of the 1,1-carboboration products (Figure 7). ${ }^{93,95-97}$ However, in the presence of an FLP, diverging reactivity pathways with alkynes exist, ${ }^{91,93,98}$ which are influenced by the steric and electronic properties of both the FLP and the substrate.

The FLP activation of terminal alkynes is postulated to proceed via the same activated alkyneborane $\sigma$-complex, which can either undergo nucleophilic attack at the Lewis acidic $\beta$-carbon to form the olefin-bridged zwitterionic salt, or be deprotonated by the external nucleophile to form a bimolecular alkynylborate salt. The reaction pathway is directed by both the steric size and basicity of the partner base, where more Brønsted basic and bulky phosphines favor deprotonation and formation of the alkynylborate product. The alkyne-borane activation $\sigma$ complex bears some resemblance to typical TM-alkyne m-complexes, which are known to rearrange similarly to form vinylidene ligands. ${ }^{99}$
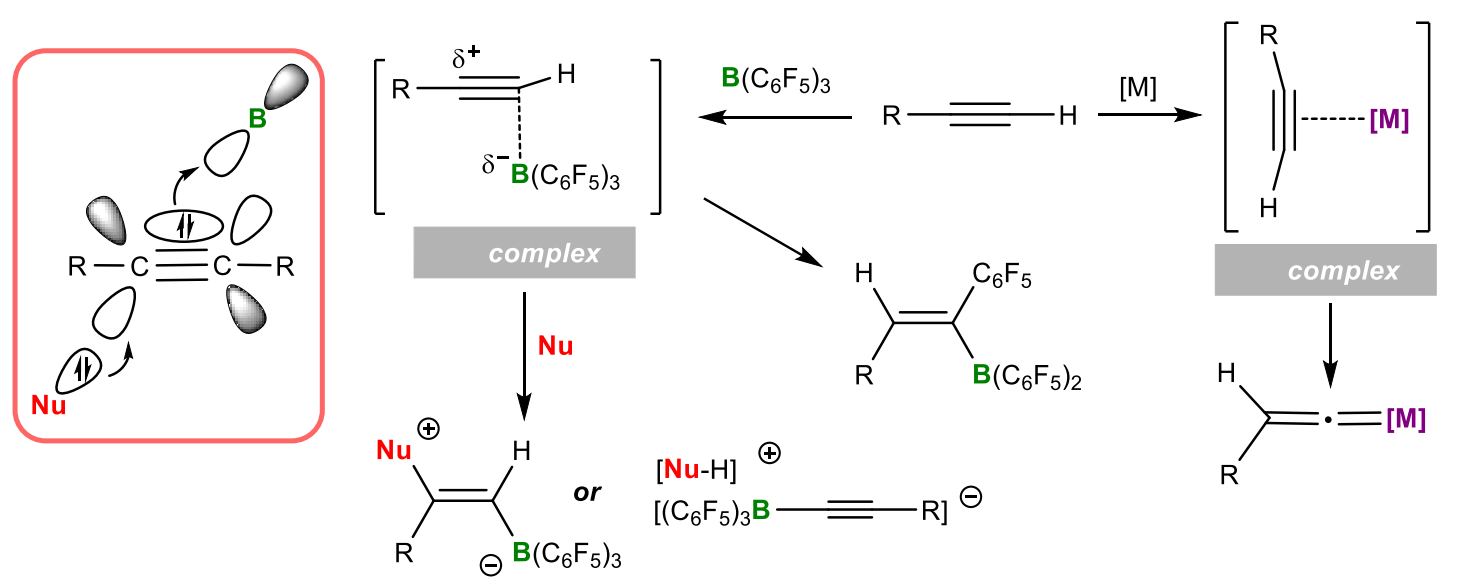

Figure 7. Typical FLP and TM reactivity with alkynes.

Alkyne activation induced by strongly Lewis acidic boranes has led to the development of stoichiometric $\mathrm{C}-\mathrm{C}$ bond forming reactions with enamines and pyrroles, ${ }^{100}$ as well as catalytic alkyne hydroamination, ${ }^{101,102}$ a transformation which is catalyzed by numerous TM complexes by similar mechanisms, ${ }^{103}$ as well as catalytic alkyne hydroboration. ${ }^{104-110}$ Subsequent work by the groups of Melen, Stephan, Paradies, and Hashmi has demonstrated that strongly Lewis acidic boranes can be utilized to induce a wealth of intramolecular cyclization reactions involving propargyl esters ${ }^{111,112}$ amides, ${ }^{113}$ and related systems where an activated alkyne is attacked by an internal nucleophile. ${ }^{114-125}$ Such reactions in many cases mirror transformations accessible typically via carbophillic Au or Pt catalysts. ${ }^{103}$ An exemplary cyclization of a 1,5 -enyne ${ }^{115}$ is 
presented in Figure 8, which may be accessed via either boron- or gold-based catalysis. Triphenylphosphine is also added in this case to act as a proton shuttle.

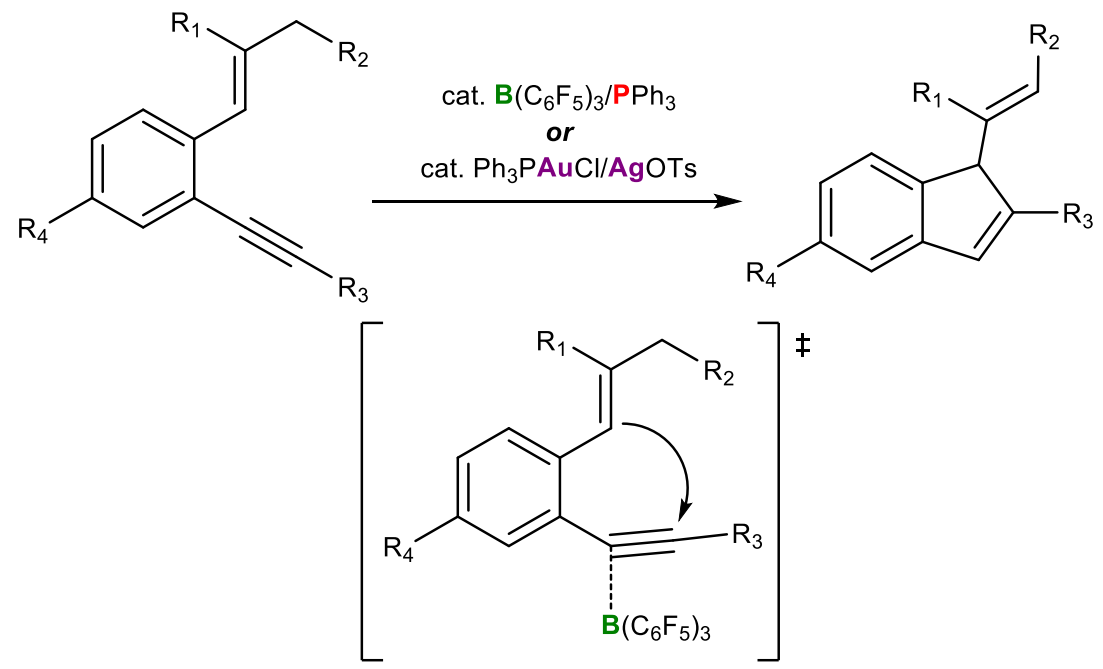

Figure 8. FLP- and TM-catalyzed 1,5-enyne cyclization

\subsection{Carbon Monoxide}

TM complexes bind $\mathrm{CO}$ via withdrawal of electron density from a C-centered sp orbital, while populating the $\mathrm{C}-\mathrm{O} \pi^{*}$-orbital, resulting in a weakening of the $\mathrm{CO}$ bond and a decrease in the $\mathrm{CO}$ vibrational frequency. Lewis acidic boranes, such as $\mathrm{BH}_{3},{ }^{126}$ and $\mathrm{HB}\left(\mathrm{C}_{6} \mathrm{~F}_{5}\right)_{2},{ }^{127,128}$ perfluoroalkylboranes ${ }^{129-131}$ and electron deficient boroles ${ }^{132}$ are also known to form weakly bound $\sigma$-complexes with $\mathrm{CO}$. However, as trivalent boron lacks non-bonding electrons to populate the $\mathrm{CO} \pi^{*}$-orbital, such species display long B-C distances and typically have increased $\mathrm{CO}$ vibrational frequencies with respect to free $\mathrm{CO}$, due to the greater electrostatic polarization of the $\mathrm{C}-\mathrm{O}$ unit upon coordination to a borane. ${ }^{133}$ Nevertheless, borane-carbonyls are susceptible to nucleophilic attack by external bases, such as $\mathrm{NH}_{3}$, at the carbonyl carbon (Figure 9; A). ${ }^{129,130}$ Such reactions follow a familiar mechanism, where withdrawal of electron density via $\sigma$-complexation acidifies the carbonyl carbon to accept electrons from an external donor. While the prototypical $\mathrm{B}\left(\mathrm{C}_{6} \mathrm{~F}_{5}\right)_{3} / \mathrm{PtBu}_{3} \mathrm{FLP}$ does not react with $\mathrm{CO}$, the ethylene-linked phosphine-borane FLP systems of Erker and coworkers do capture $\mathrm{CO}$ to form five-membered phosphonium formylborate complexes (Figure 9; B), ${ }^{134,135}$ as do related B/N intermolecular FLPs. ${ }^{136}$ Interestingly, X-ray structures revealed that the coordination of such species is markedly unsymmetrical, with large B-C-O angles $\left(\approx 135^{\circ}\right)$, long P-C distances $(2.04-2.10 \AA)$, and short $\mathrm{C}-\mathrm{O}$ distances (1.17-1.19 $\AA$ ). Such features suggest a bonding environment analogous to TM-carbonyl compounds, where the phosphine donates electron density into the $\Pi^{*}$-orbital of a formal C-O triple bond. The IR stretching frequencies of such species are in the range of $1757-1791 \mathrm{~cm}^{-1}$, indicating strong activation relative to free CO. Trifunctional FLP systems have also recently been applied in the capture of CO. ${ }^{137}$ 

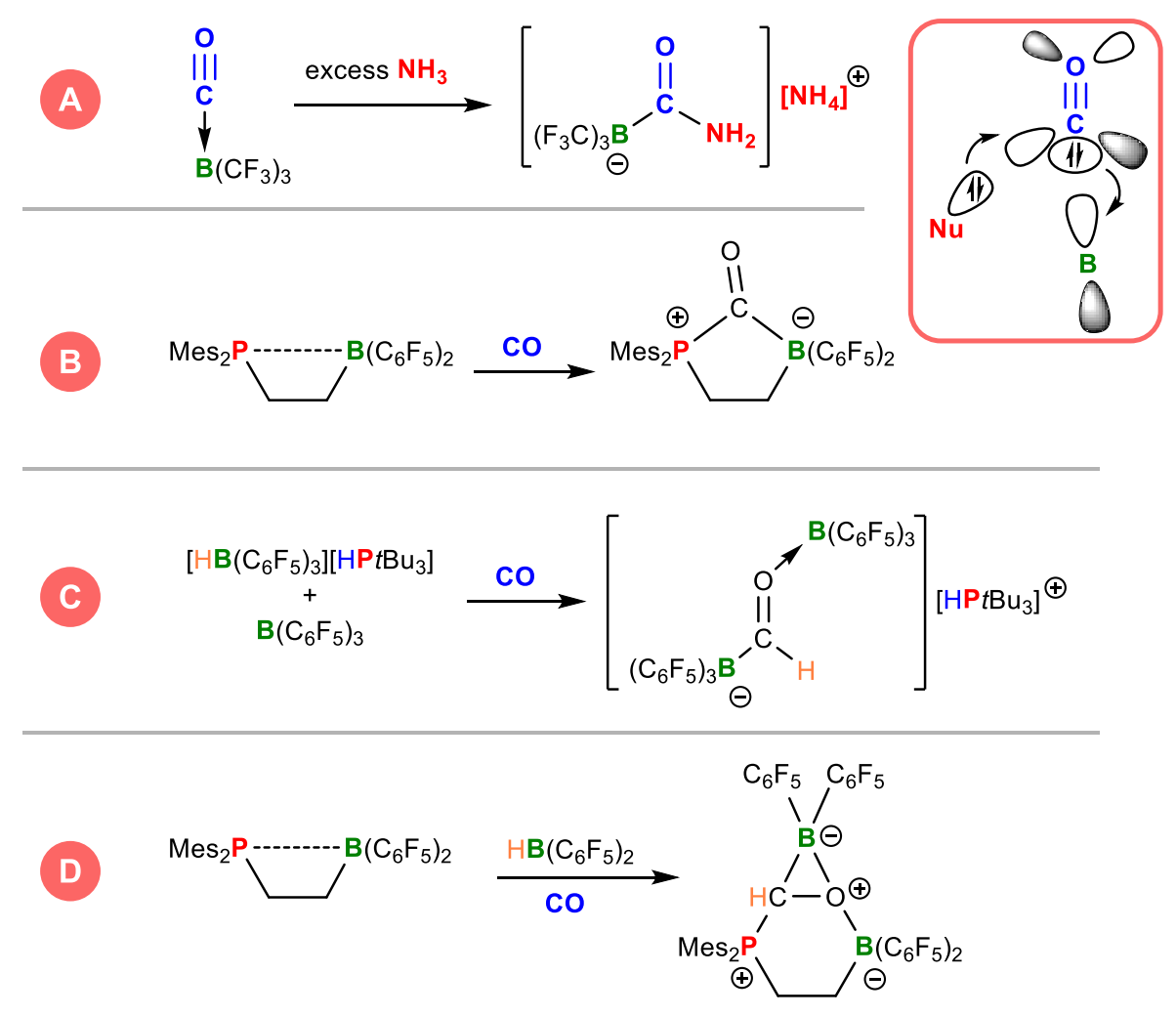

Figure 9. CO capture and insertion with FLP systems.

Insertion reactions of $\mathrm{CO}$ into the $\mathrm{B}-\mathrm{C}$ bonds of simple alkylboranes have long been known, and have found application in the synthesis of alcohols, aldehydes, and ketones. ${ }^{138}$ Such reactions are reminiscent of metallo-acyl intermediates derived from $\mathrm{CO}$ insertion into metal-alkyl complexes relevant to hydroformylation catalysis. ${ }^{139}$ However, $\mathrm{CO}$ insertion reactions typically do not proceed with simple hydridoboranes in the absence of additional reagents. ${ }^{128}$ Considering the relevance of such transformations to Fischer-Tropsch catalysis, ${ }^{140,141}$ efforts were undertaken to apply an FLP approach to this problem. Stephan and coworkers have demonstrated the use of a $\mathrm{H}_{2}$-derived borohydride as the base in a FLP system, leading to the trapping of the $\mathrm{CO}$ by 1,1-insertion into the $\mathrm{B}-\mathrm{H}$ bond (Figure 9; $\mathrm{C}$ ), whereupon further hydrogenation and insertion reactions involving the formylborate unit can occur. ${ }^{142-144}$ Similarly, Erker and coworkers have demonstrated that CO-hydridoborane adducts also react with FLPs with $\mathrm{CO}$ insertion into the $\mathrm{B}-\mathrm{H}$ unit to yield formylborate complexes (Figure 9; D), ${ }^{127,128,145-147}$ which have also been used in further $\mathrm{C}-\mathrm{C}$ - and $\mathrm{C}-\mathrm{N}$-bond-forming reactions. ${ }^{148}$ Despite the demonstration of these stoichoimetric reductions, the catalytic use of $\mathrm{CO}$ as a feedstock in FLP transformations has not yet been realized, likely due to the strong bonds often formed between Lewis acidic boranes and oxygen-containing functional groups.

\subsection{Arene $\mathrm{C}-\mathrm{H}$ activation}

The selective $\mathrm{C}-\mathrm{H}$ functionalization of $\mathrm{C}-\mathrm{H}$ bonds in arenes and alkanes is one of the most challenging fields of transition metal catalysis. The last few decades have seen an explosion of interest in developing different approaches to $\mathrm{C}-\mathrm{H}$ bond activation. These investigations have 
given rise to a whole field of research focused on the cleavage and functionalization of the $\mathrm{C}-\mathrm{H}$ bond by transition metals.

While stoichiometric $\mathrm{C}-\mathrm{H}$ borylation reactions have been reported with a number of highly Lewis acidic boranes and borenium ions, ${ }^{61,149-159,160}$ these processes employ stoichiometric amounts of highly reactive reagents through a Wheland-like mechanism. In a few cases, Friedel-Crafts-type catalysis could be observed for the TM-free catalytic borylation of arenes, in mechanisms that bear little similarity to TM chemistry. ${ }^{152,154,158,161-163}$ Similar reactions are typically metal catalyzed, ${ }^{164,165}$ often by precious metals such as iridium or palladium, which act in tandem with an internal base.

Nevertheless, here too can FLPs show their metallomimetic behavior. While select TM systems can selectively cleave $\mathrm{C}-\mathrm{H}$ bonds at a single atom, Fontaine and coworkers have shown a similar process between proximal $\mathrm{B}$ and $\mathrm{N}$ atoms in ambiphilic amino-borane FLP complexes such as 3. ${ }^{166,167}$ In this system, $\mathrm{C}-\mathrm{H}$ bonds from heteroarenes are first activated at the FLP site by simultaneous coordination to the boron centre and deprotonation of the arene $\mathrm{C}-\mathrm{H}$ functionality (Figure 10, TS), ${ }^{168}$ followed by elimination of $\mathrm{H}_{2}$. This bond activation can be followed by $\sigma$-bond metathesis with pinacolborane, enabling a catalytic cycle for the borylation of heteroaromatic $\mathrm{C}-\mathrm{H}$ bonds under mild conditions. A bench-stable pre-catalyst activated by the addition of pinacol borane has also been reported. ${ }^{169}$ Repo and co-workers later extended this activation to include simple arenes and alkenes using a similar system, albeit in a stoichiometric process. ${ }^{170}$ 


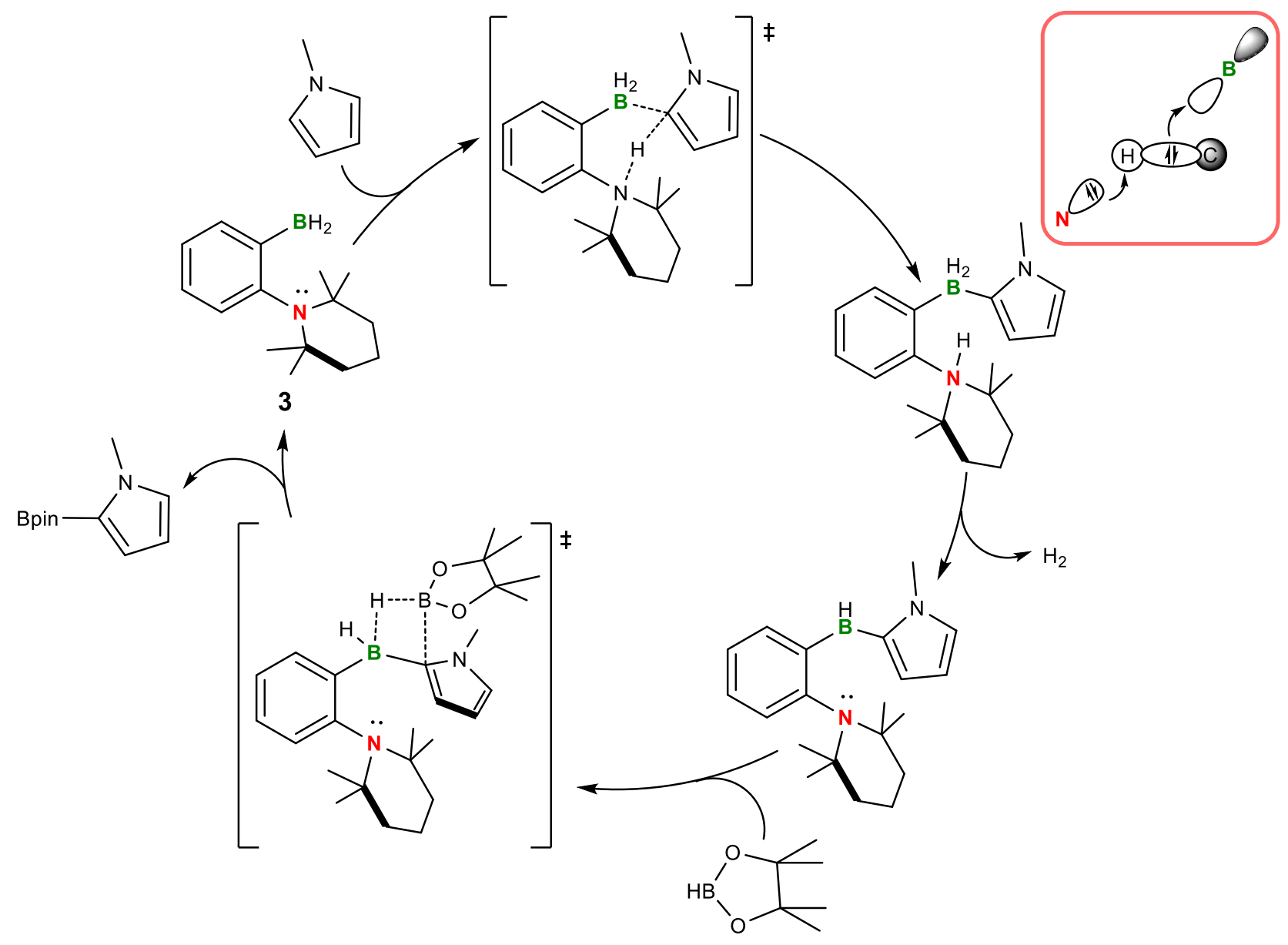

Figure 10. FLP-mediated heteroarene $\mathrm{C}-\mathrm{H}$ borylation.

\subsection{Summary}

The combination of an electron-deficient borane with another atom that provides a non-bonding electron pair of electrons can be used to effect many transformations typically exclusive to transition metal systems, such as the activation and delivery of $\mathrm{H}_{2}$ to unsaturated substrates, $\mathrm{CO}$ binding, alkyne hydroamination, and $\mathrm{C}-\mathrm{H}$ functionalization. While often metals achieve simultaneous donation and withdrawal of electrons from a substrate due to their single-site behavior, the binary nature of many FLPs requires pre-organization of the reaction partners, which may be in the form of a FLP 'encounter complex' $\left(\mathrm{H}_{2}\right)$, or pre-association of the substrate with one FLP component via formation of a van der Waals complex (olefins) or a $\sigma$-complex ( $\mathrm{CO}$, alkynes), followed by reaction of the activated complex with the other FLP partner.

\section{Metallomimetic reactivity through two boron atoms}

While catalytic breakthroughs have been achieved through inter-element cooperativity, metallomimetic behavior can also be attained using only combinations of boron-based active centers. In recent years, unusual dinuclear boron complexes have shown reactivity towards the 
binding of alkynes, dihydrogen and carbon monoxide, in manners that are reminiscent of transition metals.

\subsection{Nonbonded boron centers}

Boron-boron cooperative reactivity was convincingly demonstrated by Kinjo and coworkers in the cases of 1,3,2,5- (4) $)^{171,172}$ and 1,4,2,5-diazadiborinine (5) ${ }^{173}$ complexes (Figure 11; A, B). These aromatic compounds react with a variety of substrates $\left(\mathrm{CO}_{2}, \mathrm{MeOTf}, \mathrm{HBpin}, \mathrm{Ar}{ }_{2} \mathrm{PH}\right.$, and others) in a manner that suggests the presence of an unusually nucleophilic boron center. Consequently, $\mathbf{4}$ and $\mathbf{5}$ possess similar ambiphilic cooperative properties that give rise to the metallomimetic reactivity in FLP systems, and also have been observed to bind alkynes and alkenes in an analogous manner. More recently, the same group showed activity of the related compound 6 towards the cleavage of $\mathrm{H}_{2}$ and $\mathrm{NH}_{3}$ (Figure $11 ; \mathrm{C}$ ). ${ }^{174}$ Similar reactivity towards ethylene was also observed with NHC-diboranthracene adduct 7. ${ }^{175}$ Interestingly, the selective 'side-on' binding of dioxygen from dry air was observed. Notably, the metallomimetic behavior of diboraanthracene complexes could be increased by two-electron reduction (Figure 11; D), ${ }^{176,177}$ where the reduced dilithiodiboraanthracenide 8-R $(R=H, C C t B u)$ complexes were shown to activate $\mathrm{H}_{2}$, as well as the $\mathrm{C}-\mathrm{H}$ bond of terminal alkenes (Figure 11; E). 

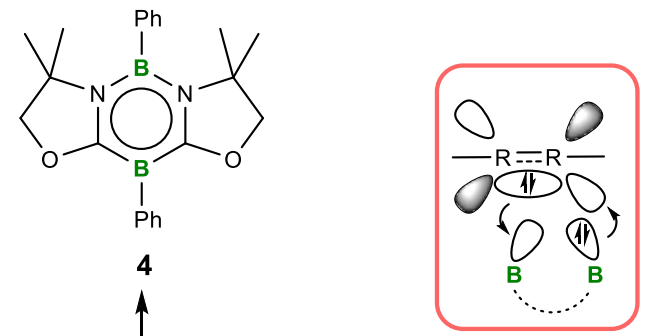

A

()

(C)

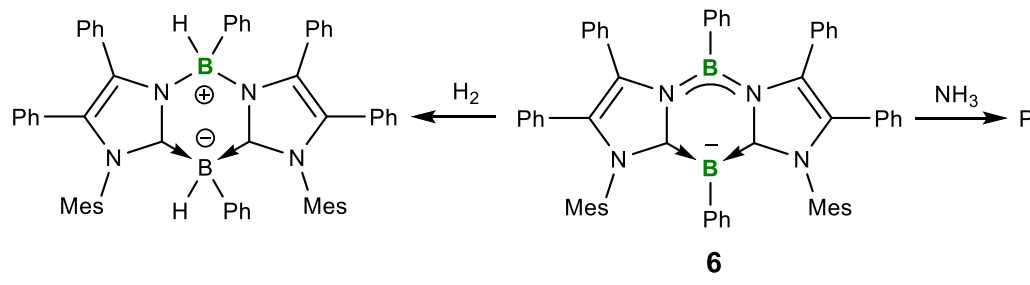<smiles>CN1C(c2ccccc2)=C(c2ccccc2)N2C1[BH-](c1ccccc1)[C@H]1N2C(c2ccccc2)=C(c2ccccc2)[B-]1(N)c1ccccc1</smiles>

D

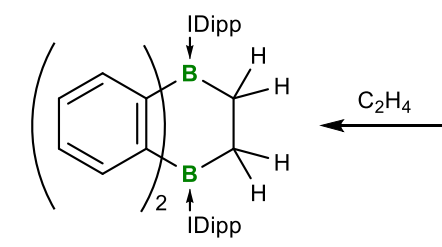

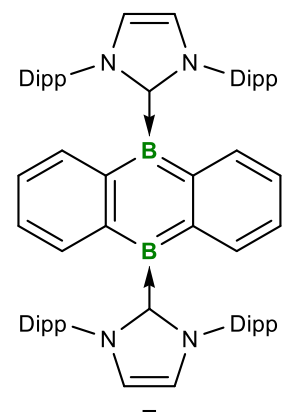

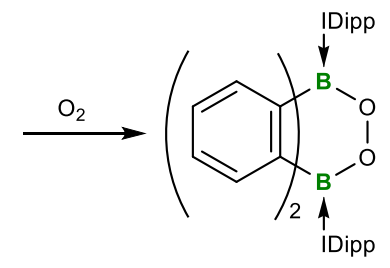

E<smiles>[R][R]B1c2ccccc2B([R])c2ccccc21</smiles><smiles></smiles> 
Figure 11. Activation of small molecules by non-bound boron centers.

\subsection{Metallomimetic chemistry of B-B bonds}

Early work on tetrachlorodiborane $\left(\mathrm{B}_{2} \mathrm{Cl}_{4}\right)$ demonstrated that this highly acidic and reactive molecule could cleave molecular hydrogen at room temperature in the absence of a catalyst. ${ }^{178}$ The products of the reactions are $\mathrm{BCl}_{3}$ and $\mathrm{B}_{2} \mathrm{H}_{6} . \mathrm{B}_{2} \mathrm{~F}_{4}, \mathrm{~B}_{2} \mathrm{Cl}_{4}$ and $\mathrm{B}_{2} \mathrm{Br}_{4}$ were also found to spontaneously add to ethylene. ${ }^{179-182}$ Since then, the diboration of many unsaturated bonds using diboranes has been established in the presence or absence of catalysts. ${ }^{183,184}$

In recent years, departing from the chemistry of classical diborane(4) systems such as $B_{2} p i n_{2}$, $\mathrm{B}_{2} \mathrm{Cat}_{2}, \mathrm{~B}_{2}(\mathrm{OMe})_{4}$ and $\mathrm{B}_{2}\left(\mathrm{NMe}_{2}\right)_{4}$, unusual complexes featuring reactive $\mathrm{B}-\mathrm{B}$ single and multiple bonds have been the object of considerable interest. Employing simple tetraaryldiboranes, Yamashita and coworkers ${ }^{185}$ have demonstrated the facile cleavage of molecular hydrogen by tetra(o-tolyl)diborane (9) under ambient conditions (Figure 12; A). Computational modeling of the reaction shows that while the high Lewis acidity of the diboron reagents activates the $\mathrm{H}-\mathrm{H}$ bond, B-to-H interactions complete the bond cleavage. Thus, through the concerted action of two boron atoms, TM-mimicking reactivity is enabled. These results give insight into previous chemistry that used diboron reagents as precatalysts for the reduction of acenes under harsh conditions. ${ }^{186,187}$ Interestingly, 9 also reacts with carbon monoxide with initial insertion of CO into the B-B bond, followed by subsequent rearrangement. ${ }^{188}$ Typical alkyl and arylboranes are usually relatively inert towards $\pi$-acceptor bases such as $\mathrm{CO}$, because they lack the filled orbitals that allow TM complexes to effect backbonding. In this case, however, the electron density of the B-B bond appears to be accepted by $\mathrm{CO}$, resulting in cleavage and metathesis to give a boroxine and a $\mathrm{C}-\mathrm{H}$ insertion product.

Proposing a similar mechanism as with tetra(o-tolyl)diborane, Wang et al. have recently reported the cleavage of $\mathrm{H}_{2}$ by the remarkably stable borylborenium complex 10 (Figure 12; B). In this unsymmetrical diboron reagent, a positively-charged boron center is the HOMO of the molecule, which can interact with the $\mathrm{H}-\mathrm{H} \sigma$-bond. The other boron atom supplies electron density to the antibonding orbitals of $\mathrm{H}_{2}$. ${ }^{189}$ Similar reactivity towards $\mathrm{H}_{2}$ was observed with the cyclic B,B'-dimesityldithienodiborane 11 (Figure 12; C). ${ }^{190}$

An unusual diboron framework was also found to cooperatively enable the activation of a $\mathrm{C}_{\mathrm{sp} 3}-\mathrm{H}$ bond of methyllithium. ${ }^{191}$ In this case the nucleophilic organolithium ensures the coordination of a $\mathrm{CH}_{3}$ group to a boron center and the reduction of the redox-active complex. The other boron atom, being proximal to the $\mathrm{CH}_{3}$ group, activates the $\mathrm{C}-\mathrm{H}$ bond via a $\sigma$-complex while the electron density from the B-B bond performs the cleavage. 


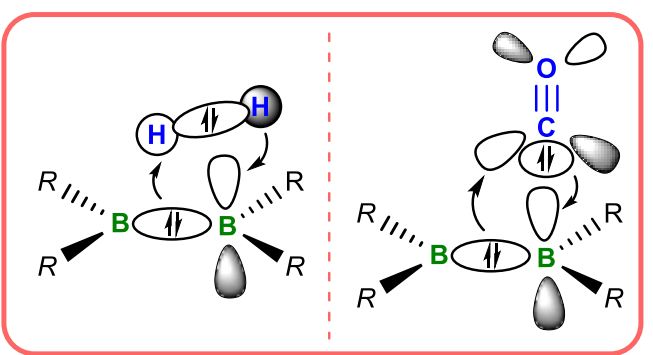

A

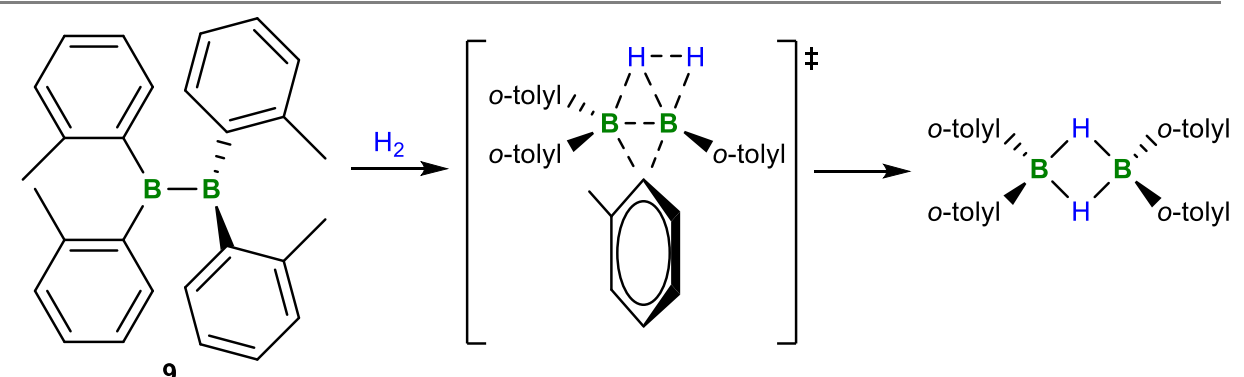

9

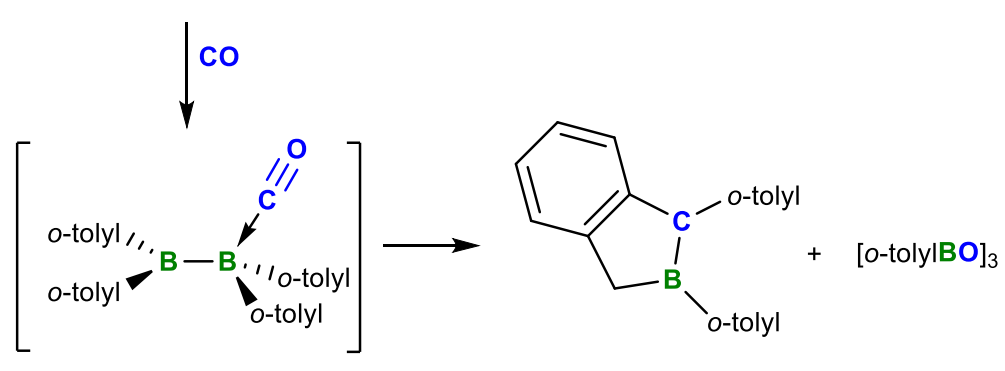

B

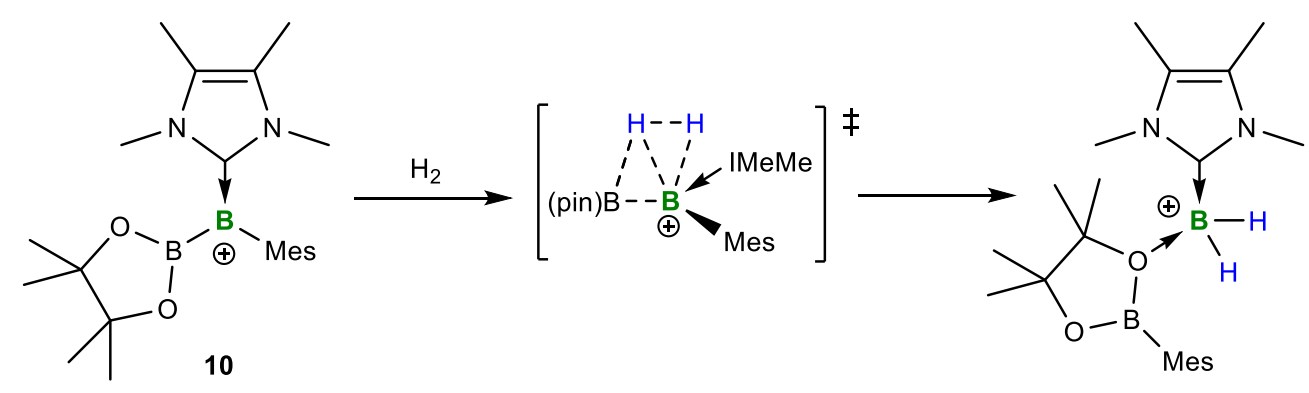

C

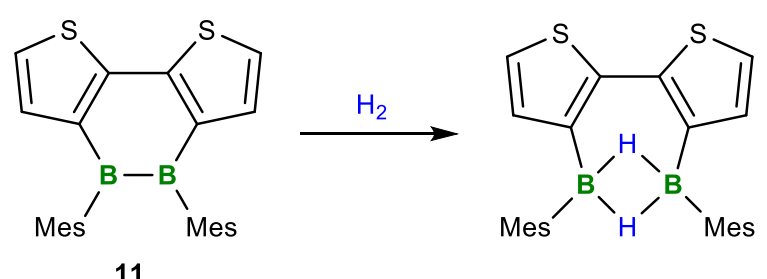

Figure 12. Reactivity of B-B single bonds towards $\mathrm{H}_{2}$ and $\mathrm{CO}$.

Molecules that feature B-B multiple bonds can display even more striking metallomimetic chemistry. Possessing boron centers in a formally lower oxidation state, they often possess 
orbitals that are ideally suited to the activation of small molecules and the breaking of relatively inert bonds. The study of such species has been the subject of many reports, beginning with the isolation of dianionic ${ }^{192,193}$ and neutral diborenes ${ }^{194,195}$ and more recently with diboryne complexes. ${ }^{196}$ As analogues of alkenes, diborenes possess a HOMO with B-B bonding of $\pi-$ symmetry and a $\pi^{*}(B-B)$ LUMO. However, the HOMO-LUMO gap is much smaller in diborenes than in ethylenes, suggesting that reactivity involving the cooperative contribution of both empty and filled orbitals is much more likely for the former. This confers a reactivity to diborene complexes that far exceeds that of their carbon-based analogs. While many diborene reactions are not formally metallomimetic - being not directly comparable with TM reactions - they most often involve an interplay of the boron-based HOMO and LUMO. Similarly to alkenes, the B=B bond in $\left[\left(\mathrm{C}_{4} \mathrm{H}_{4} \mathrm{~S}\right) \mid \mathrm{IMeB}\right]_{2}$ was found to be readily hydroborated. ${ }^{197}$ In contrast to alkenes, however, the same compound was found to react with elemental sulfur under mild conditions to effect complete B-B bond cleavage. ${ }^{198}$ With the silylated derivative $\left[\left\{\mathrm{C}_{4} \mathrm{H}_{3}(\mathrm{SiMe})_{3} \mathrm{~S}\right\} \mathrm{MeB}\right]_{2}$, more selective reactivity was achieved with $S_{8}$ and $S e$ to give diboradithiirane complexes. ${ }^{199,200}$ For their part, diborynes have been most commonly described as possessing a B-B triple bond, being isoelectronic with alkynes, but again with a narrower HOMO-LUMO gap. Even more than diborenes, diborynes species possess a rich reactivity, including interactions with chalcogens, dichalcogenides, ${ }^{199,201}$ isocyanides ${ }^{202}$ and acetone. ${ }^{203}$

In contrast to diborenes, the metallomimetic reactivity of diborynes is more clearly exemplified. Indeed, [IDippB] 2 (IDipp = 1,3-bis(2,6-diisopropylphenyl)imidazol-2-ylidene) (12) reacts rapidly with one equivalent of $\mathrm{CO}$ to give bridging adduct 13 (Figure 13, B). ${ }^{204}$ In this complex, a very long C-O bond distance (1.249(2) $\AA$ ), as well as an IR band at $1926 \mathrm{~cm}^{-1}$ indicate the strong $\pi-$ overlap of the B-B orbitals into the antibonding orbital of carbon monoxide. The addition of an excess of carbon monoxide to $[\mathrm{IDippB}]_{2}$ led to the coupling of four equivalents of $\mathrm{CO}$ into complex 14, an unprecedented reactivity mode for both boron compounds and for light maingroup elements.

Interestingly, when 1,3-bis(2,6-diisopropylphenyl)imidazolin-2-ylidene (SIDipp) ${ }^{205}$ and N-(2,6diisopropylphenyl)-2,2,4,4-tetramethylpyrrolidin-5-ylidene $\left({ }^{\mathrm{Me}} \mathrm{CAAC}\right)^{206}$ analogues of $\mathrm{NHC}$ stabilized diborynes (15 and 18, respectively) were synthesized, it was found that the $\pi-$ accepting capabilities of these ligands deplete the B-B triple bond HOMO and give rise to an electron deficient diboracumulene-type structure, where the B-B bond order decreases with an increased $\pi$-accepting character of the ligand (Figure 13, A). The reactivity of these complexes with carbon monoxide reflects this change in properties. Instead of $\mathrm{CO}$ coupling, the addition of excess CO to 18 selectively affords a bis-CO adduct (19). In this complex, the CO bond distances $(1.173(2) \AA$ ) are notably shorter than in the case of 13 , while the IR band is at a similar wavenumber $\left(1926 \mathrm{~cm}^{-1}\right) .18$ also reacts with $t$ BuNC to form a similar complex. ${ }^{202}$ In carbon monoxide chemistry, as in bonding and structure, 15 has intermediate reactivity between 12 and 18. While 16 - an analog of 19 - can be observed at $-30{ }^{\circ} \mathrm{C}$ following the addition of $\mathrm{CO}$ to 15 , gradual conversion to the coupled product 17 takes place at room temperature. 

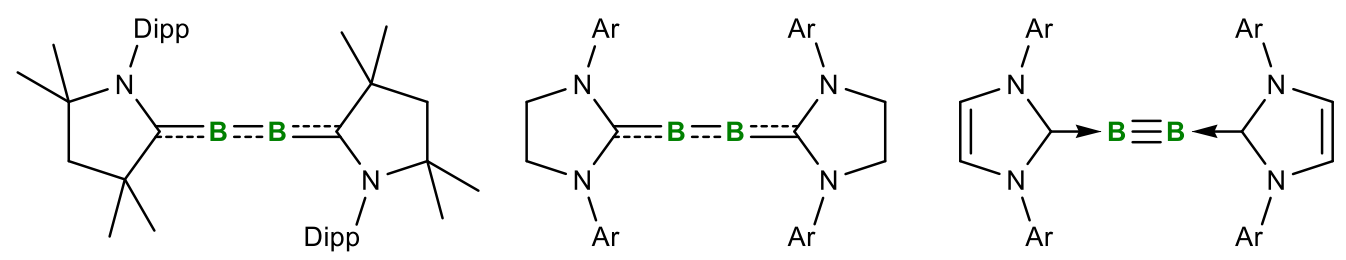

Increasing -acidity, decreasing triple bond character

B

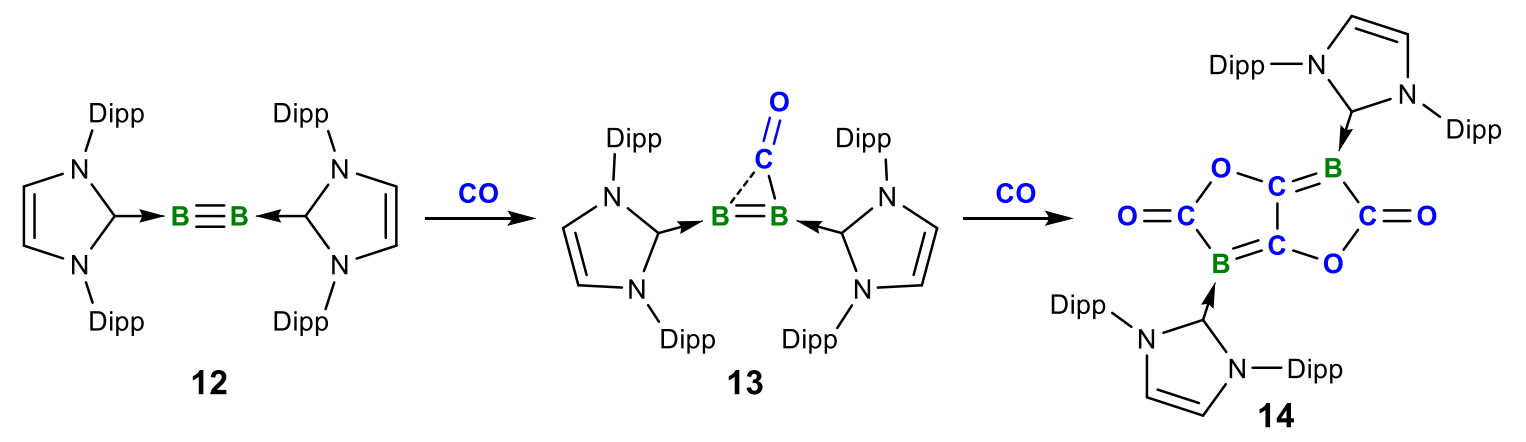

c
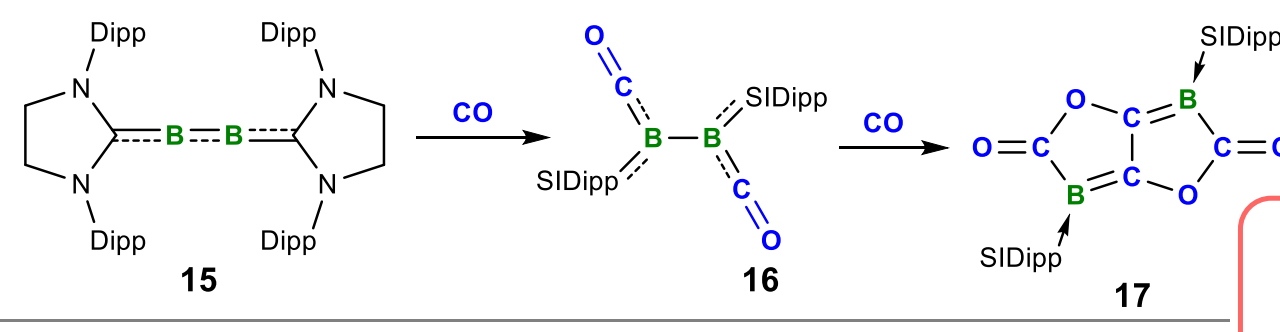

15

16

17

D<smiles>[B]C1N(O)C(C)(C)CC1(C)C</smiles><smiles>CB=C1N([Pb])C(C)(C)CC1(C)C</smiles>

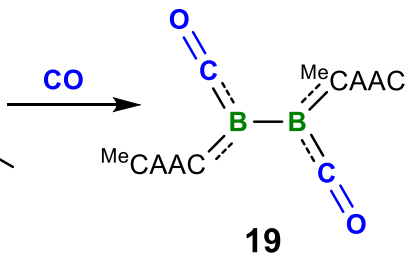

$\underset{150^{\circ} \mathrm{C}}{\stackrel{\mathrm{CO}}{\longrightarrow}}$ No reaction

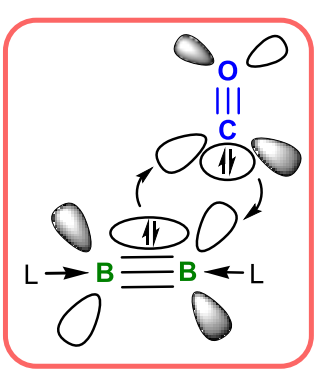

Figure 13. Diborynes, diboracumulenes and their reactivity with $\mathrm{CO}$.

With molecular hydrogen, unsaturated $\mathrm{NHC}$-stabilized diborynes and [SIDipp $\mathrm{B}_{2}$ ] were found to be unreactive. However, 18, as well as the less bulky [SIDepB] $]_{2}$ (SIDep = 1,3-bis(2,6diethylphenyl)imidazolin-2-ylidene), react spontaneously to give the corresponding trans-1,2dihydrodiborenes (Figure 14). ${ }^{207}$ The greater m-accepting character of CAAC and saturated NHCs seems to be essential for this process, with excessive steric bulk - as in the case of SIDipp - being detrimental. Interestingly, the m-acidity of CAAC and the cumulene character of 18 makes each of its boron centers isoelectronic to donor-acceptor-stabilized borylene complexes (see below), which explains their increased reactivity. The reaction of 18 with $\mathrm{H}_{2}$ is complete within a day at room temperature, while it takes two days at $80{ }^{\circ} \mathrm{C}$ for [SIDepB] $]_{2}$ to be completely hydrogenated, showing the correlation between the $\pi$-acidity of the ligand and reactivity. The mechanism of the reaction, involving the formation of a $\mathrm{H}_{2}-\mathrm{B} \sigma$-complex and 
bond-cleaving backdonation from the B-B system electron is reminiscent of the ambiphilicity of TM orbitals and the $\mathrm{H}_{2}$ activation by diborane complexes.

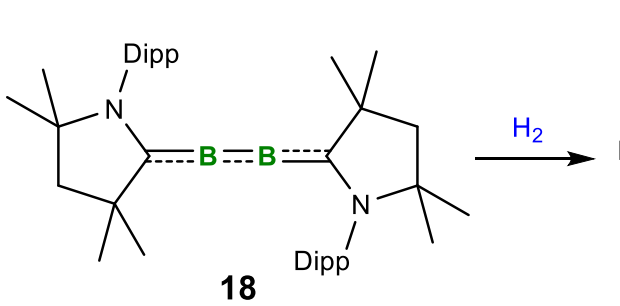

18

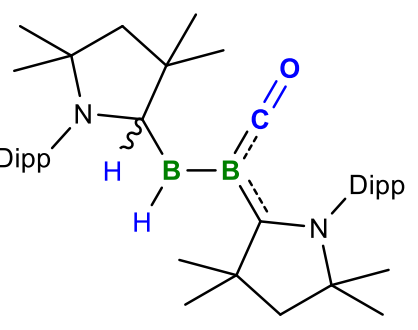

$21 b$

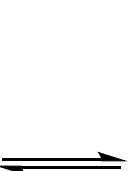

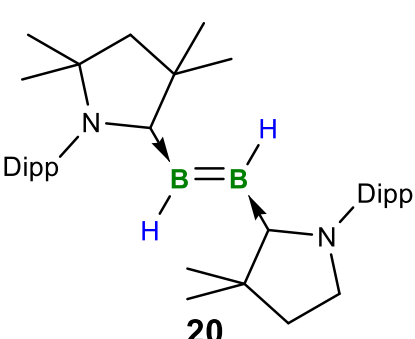

20

co
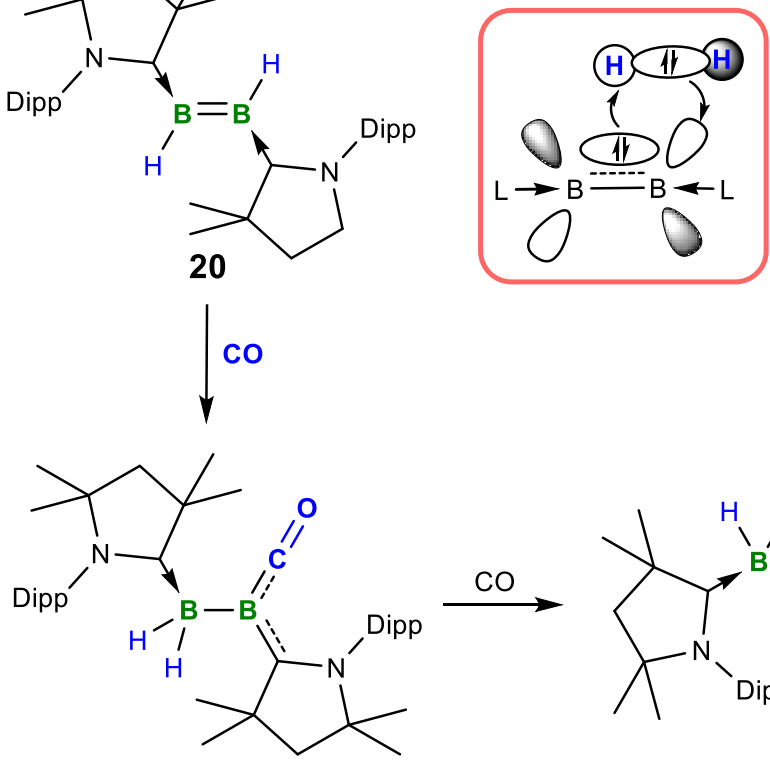

21a

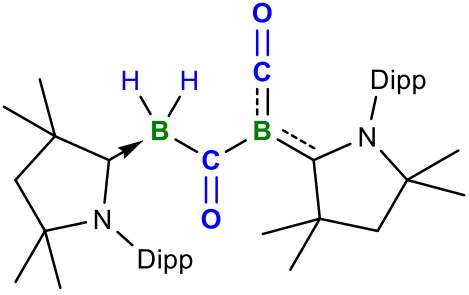

22

Figure 14. Reactivity of a diboracumulene with $\mathrm{H}_{2}$ and CO.

Notably, dihydrodiborene $\mathbf{2 0}$ is the only currently known example of a diborene that reacts with carbon monoxide (Figure 14). ${ }^{208}$ Upon treating compound 20 with CO, the formation of 21a and 21b was observed. These two complexes are isomers that depend on the transfer of a hydride back and forth from a boron center to the ${ }^{\mathrm{Me}} \mathrm{CAAC}$ ligand, and that, while being in an equilibrium in solution, could be crystallized independently. 21a and 21b are associated with IR CO stretching bands at 1928 and $1962 \mathrm{~cm}^{-1}$, respectively. A second equivalent of CO can react with 21 and insert into the B-B bond, forming compound 22, which also features $\mathrm{CO}$ complexation at boron.

While 18 reacts selectively with one equivalent of dihydrogen, ${ }^{209}$ it was also found that its coordination to one equivalent of MeIMe (1,3,4,5-tetramethylimidazol-2-ylidene) - which breaks the symmetry of the diboracumulene to give complex 23 - increases its reactivity (Figure 15). In this bound state, 23 reacts rapidly with two equivalents of molecular hydrogen in a process that completely cleaves the B-B bond. The products of the reaction are thus $\left[{ }^{\mathrm{Me}} \mathrm{CAAC}\left({ }^{\mathrm{Me}} \mathrm{IMe}\right) \mathrm{BH}\right]$ (24) and $\left[{ }^{\mathrm{Me}} \mathrm{CAACBH}_{3}\right]$ (25). Computational analysis ascribes the increased reactivity of 23 to the strong localization of the frontier orbitals on the dicoordinate boron center, suggesting a borylene type structure. Compound 23 also reacts with carbon monoxide to form a strong adduct (26) (Figure 15). The CO stretching frequency of $v=1912 \mathrm{~cm}^{-1}$ in the resulting complex suggests extremely strong B-to-CO backdonation. This backdonation is further supported by the long $\mathrm{C}-\mathrm{O}$ bond distance $(1.170(2) \AA)$ as well as by computational modelling. 


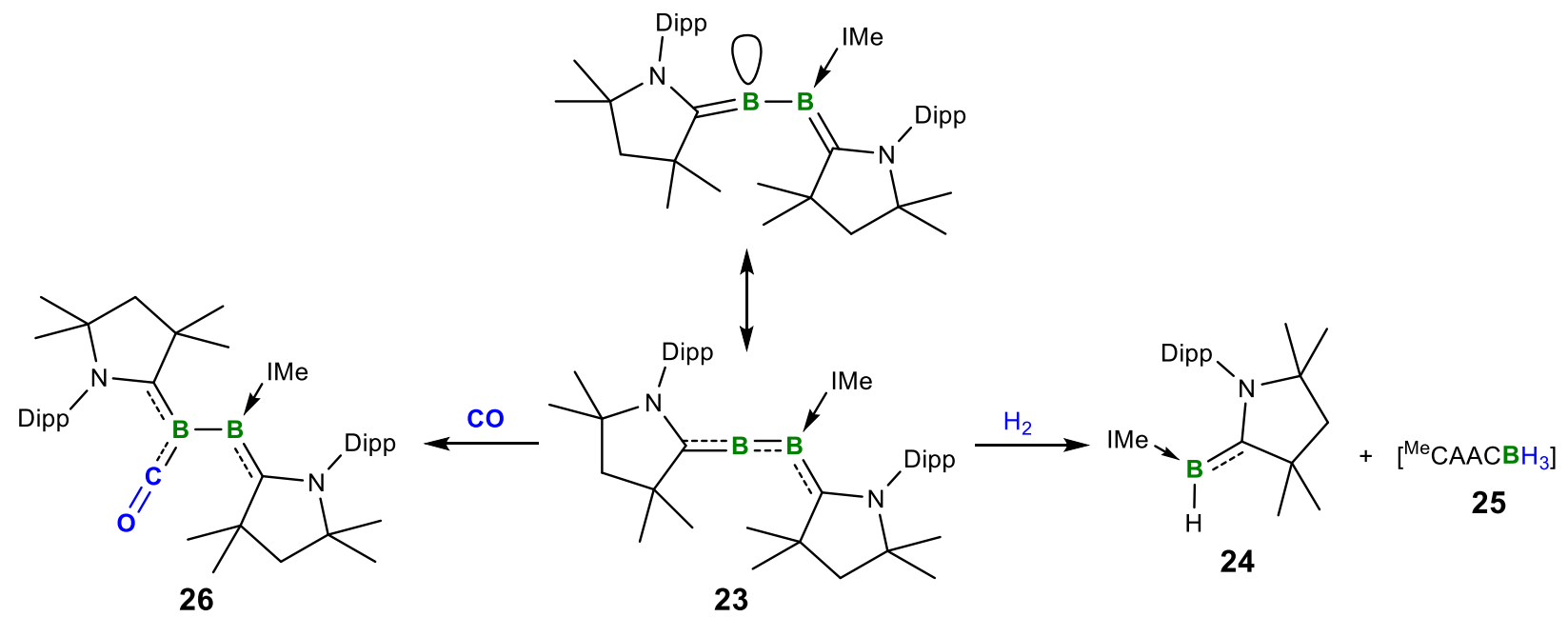

Figure 15. Reactivity of an NHC-coordinated diboracumulaene with $\mathrm{H}_{2}$ and $\mathrm{CO}$.

Metallomimetic reactivity is also found in the interaction of phosphine-stabilized diborenes and $\left[\left({ }^{\mathrm{Me}} \mathrm{CAAC}\right) \mathrm{B}\right]_{2}$ with alkynes. While organic alkenes and alkynes do not spontaneously undergo $[2+2]$ additions, transition-metal carbene complexes (featuring a $M-C$ double bond), as well as heavy main-group multiple bonds, undergo such metathesis reactions. In work from our laboratories, diborene $\mathbf{2 7}$ and the diboracumulene $\mathbf{1 8}^{210}$ where shown to react with a range of acetylenes (Figure 16). While the reaction with $\mathbf{2 7}$ is performed under photolytic conditions presumably in order to overcome symmetry constraints - and thus has precedence in conventional alkene chemistry, ${ }^{211}$ the reaction of $\mathbf{1 8}$ with acetylene and propyne is possibly spontaneous from the ground state. The products of the reaction differ based on the substrate, presumably on steric grounds: while exposure of $\left[\left({ }^{\mathrm{Me}} \mathrm{CAAC}\right) \mathrm{B}\right]_{2}$ to acetylene leads to the formation of neutral diborabenzene 28 , a similar reaction with $\mathrm{MeCCH}$ leads to the complete cleavage of the $\mathrm{C}-\mathrm{C}$ triple bond and the formation of the cyclic diradicaloid 29. In both cases, an initial [2+2] cycloaddition is expected to be the first step of the reaction. Such a process is somewhat metallomimetic in nature because it involves the interaction of the alkyne HOMO with a boron-centered orbital and the backdonation of a B-B orbital into the C-C $\pi^{*}$. However, the resulting diboracyclobutene $\mathbf{3 0}$ is unstable and can undergo a second [2+2] cycloaddition with acetylene or a rearrangement to the thermodynamically stable 1,3 isomer. ${ }^{212,213}$ The formation of dimetallacyclohexadienes from alkynes is also a staple of TM chemistry. ${ }^{214}$ 

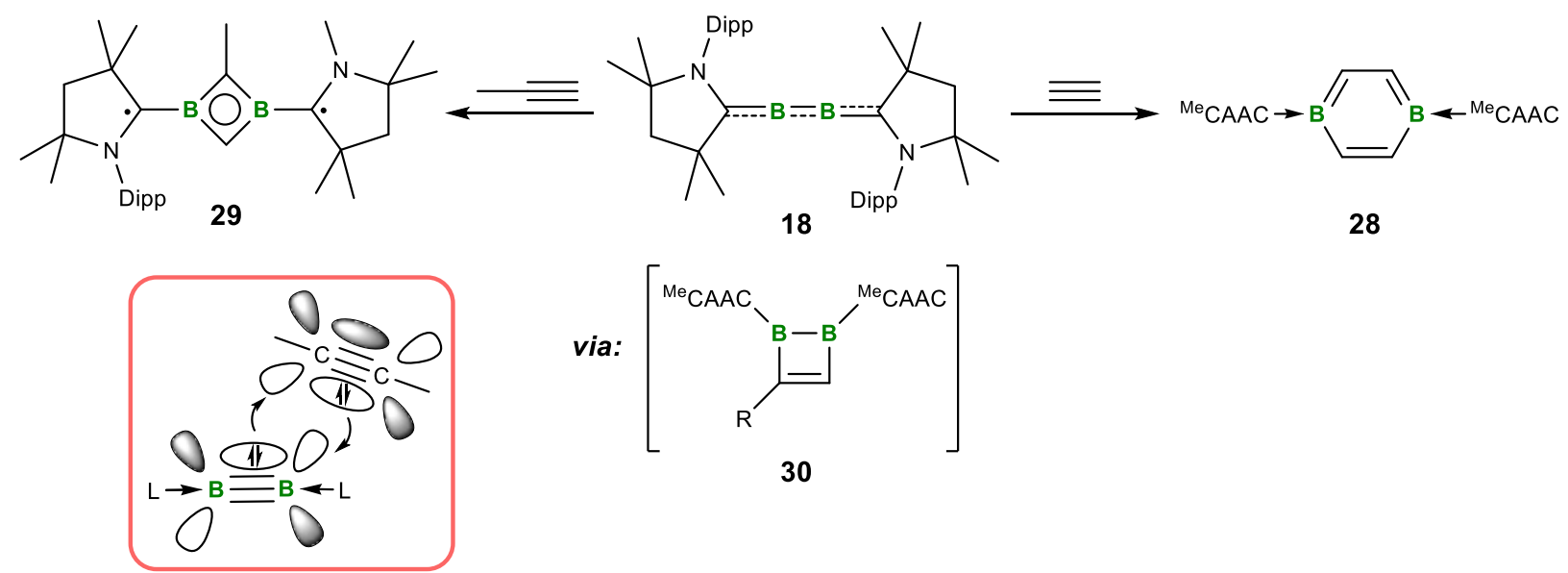

Figure 16. Reactivity of a diboracumulene with alkynes.

\subsection{Summary}

Metallomimetic chemistry can be attained by the cooperative action of two boron centers. Interestingly, in certain molecules, two remote boron atoms can act together to activate small molecules in a manner that is reminiscent of FLPs and of the ambiphilicity of TM complexes. In such transformations, one of the boron atoms appears to be reacting as a nucleophile which, combined a Lewis acidic boron center, achieves the side-on binding of alkene and alkynes and the cleavage of $\mathrm{H}_{2}$ among others.

B-B single and multiple bonds are also found to be involved in a rich variety of TM-like reactivity. The availability of the electron density from the B-B bond to be shared to substrates in $\pi-$ backdonnation allows some diboranes, diborenes, diborynes and diboracumulenes to activate $\mathrm{CO}, \mathrm{H}_{2}$, alkynes and other small molecules through TM-like electronic processes.

\section{Metallomimetics centered around a single boron atom 3.1. Boron(III) systems}

A unique challenge is associated with obtaining transition-metal-like properties in complexes that feature a single boron active site and that do not provide cooperative reactivity from another heteroatom. In such cases, the combination of filled and empty orbitals that is required for metallomimetic reactivity have to be provided by a single boron atom. However, a single boron atom in its favored oxidation state $(+3)$ is unlikely to be the center of both the HOMO and LUMO of a molecule. In a few examples, this deficiency can be countered by the use of highly Lewis acidic boranes, which have been shown to be sufficient for the activation of nonpolar substrates such as dihydrogen under mild conditions. In these unique systems, the high electrophilicity of the boron centers weakens the $\mathrm{H}-\mathrm{H}$ bond sufficiently to enable a $\sigma$-bond metathesis with alkyl substituents that would otherwise require harsh conditions. ${ }^{186,187,215,216}$ Boranes that have been shown to be sufficiently acidic to activate dihydrogen in this manner under mild conditions are pentafluoroaryl and pentaaryl boroles and their derivatives (Figure 17; 
$\mathrm{A}, \mathrm{B}),{ }^{217-220}$ as well as $\mathrm{HBAr}_{2}\left(\mathrm{Ar}^{\mathrm{F}}=2,4,6\right.$-tris(trifluoromethyl)phenyl) or $\mathrm{HB}\left(\mathrm{C}_{6} \mathrm{~F}_{5}\right)_{2}$ (Figure 17; C). ${ }^{221}$ Interestingly, while the reactivity of $\mathrm{HBAr}_{2}$ with hydrogen was only proven using $\mathrm{H} / \mathrm{D}$ exchange, the less bulky $\mathrm{HB}\left(\mathrm{C}_{6} \mathrm{~F}_{5}\right)_{2} \mathbf{3 1}$ was recently found to act as a metal-free catalyst for the hydrogenation of alkenes under mild conditions via an hydroboration - hydrogenolysis mechanism (Figure 18). ${ }^{84}$ The authors further determined computationally that the $\sigma$-bond metathesis of $\mathrm{H}_{2}$ occurs via donation of electron density of the $\sigma$-orbital of $\mathrm{H}_{2}$ to the vacant $p$ orbital of the Lewis acidic borane, and donation of electron density of the $\mathrm{C}_{\text {alkyl}}-\mathrm{B} \sigma$-orbital to the $\sigma^{*}$-orbital of $\mathrm{H}_{2}$, and subsequent asynchronous formation of the $\mathrm{B}-\mathrm{H}$ and $\mathrm{C}-\mathrm{H}$ bonds avoids the formally forbidden [2+2] transformation. Such hydrogenations are mechanistically identical to hydrogenations reported with $\mathrm{d}^{0} \mathrm{TM}$ complexes, ${ }^{222}$ and very similar to hydrogenations based on transition metals such as $\mathrm{Ru}(\mathrm{II})$, where formal oxidative addition of $\mathrm{H}_{2}$ is disfavored. ${ }^{223} \mathrm{~A}$ related mechanism is postulated for the hydroboration of alkenes catalyzed by hydro(3,5bis(trifluoromethyl)phenyl)boranes. ${ }^{224}$

A<smiles>C[Al]C1=C([Al])C(Br)=C([Al])B1[Al]</smiles>

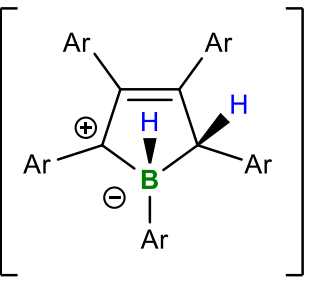

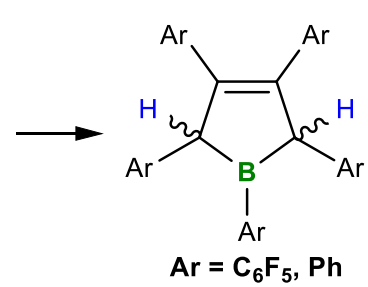

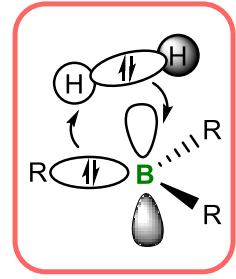

B

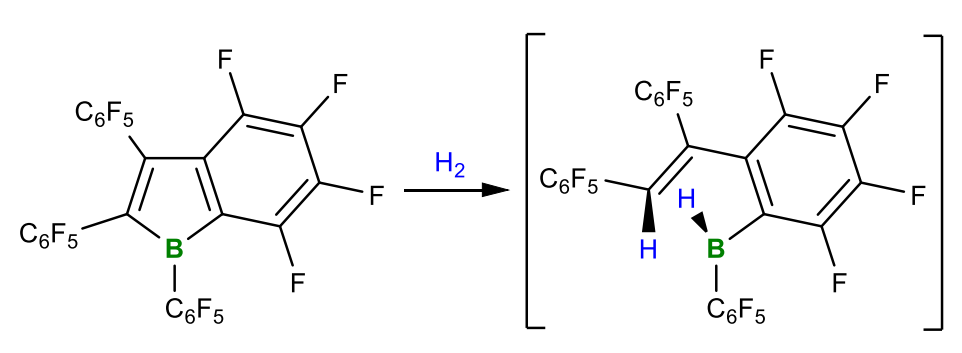<smiles>CC1CCCCC1C(=C(C(F)(F)F)C(F)(F)F)c1c(F)c(F)c(F)c(F)c1P(F)(F)(F)F</smiles>

C

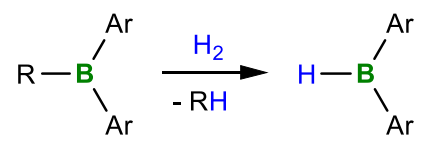

$\operatorname{Ar}=A r^{F}, R=H$

$A r=C_{6} F_{5}, R=H$, alkyl (31)

Figure 17. Reaction of electron-deficient boron(III) systems with $\mathrm{H}_{2}$. 


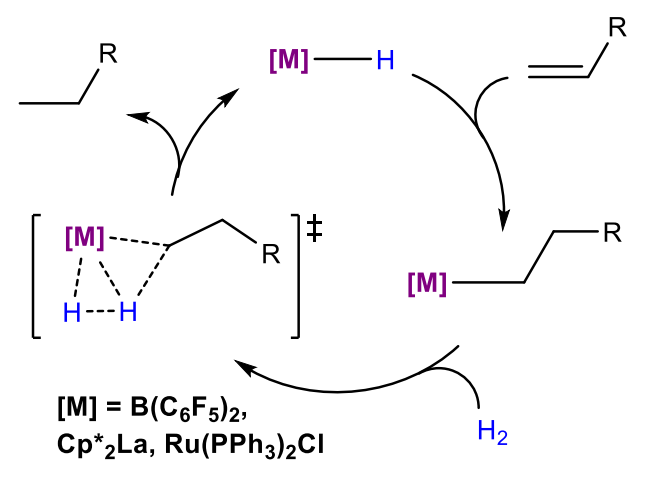

Figure 18. $\sigma$-Bond metathesis mechanism of olefin hydrogenation by 31 and other TM species.

Confinement of boron(III) to an aromatic heterocycle can also be used to effect metallomimetic reactivity, enabling the binding of $\pi$-acceptor ligands that usually only interact with TM complexes. For example, in free borabenzene, the strong Lewis acidity of the boron center corresponds to an empty $\mathrm{sp}_{2}$ orbital, and the HOMO is associated with the delocalized $\pi-$ electrons within the aromatic system. ${ }^{225}$ The reactivity conferred by this electronic environment is notably revealed by the ability of borabenzene to bind dinitrogen - a molecule that usually does not react with non-metal elements - under matrix isolation conditions to give adduct 32 (Figure 19, A). ${ }^{226}$ Subsequent computational work ${ }^{225}$ revealed the importance of $\pi$-backdonation from the aromatic ring of borabenzene to suitable orbitals in $\mathrm{N}_{2}$ and various other ligands. It was likewise predicted that $\mathrm{CO}$ should bind borabenzene very strongly, despite the corresponding adduct (33) having never been reported (Figure 19, B).

A<smiles></smiles>

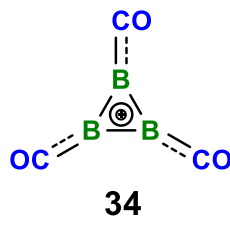<smiles>N=[B-]1[P-](=N)[P-]1=N</smiles>

32

$$
O C=B=B=C O
$$

36

Detected in matrix-isolation or in the gas phase<smiles>O=[Sb]1=CC=CC=C1</smiles>

33

Theroretically predicted
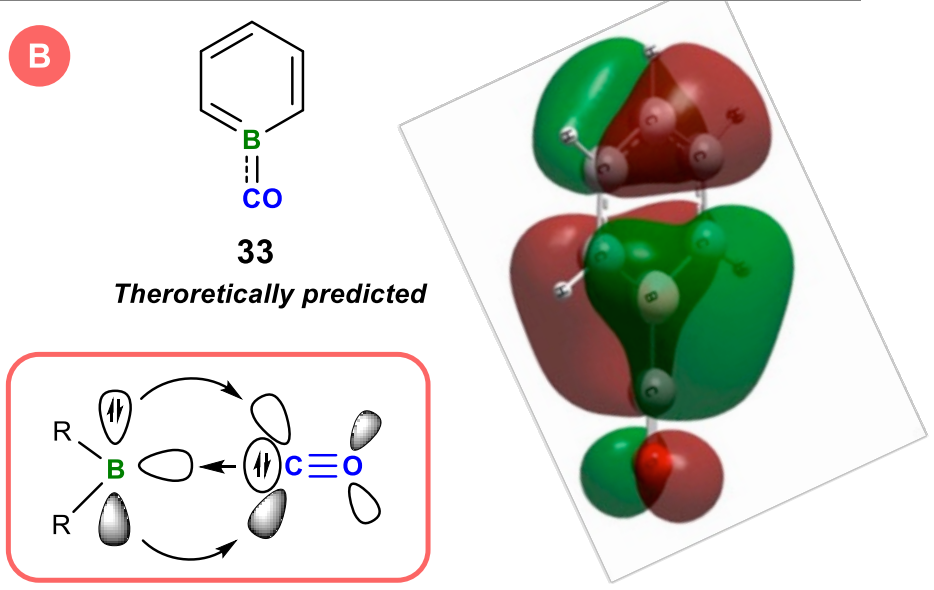
Figure 19. Aromatic B-containing cycles featuring bound $\mathrm{N}_{2}$ and $\mathrm{CO}$. The $\mathrm{HOMO}$ of 33 is depicted in the orbital diagram.

Similarly, $\mathrm{B}_{3}(\mathrm{CO})_{3}{ }^{+}(34) \mathrm{B}_{3}\left(\mathrm{~N}_{2}\right)^{+}(35)$ and cations have been observed in the gas phase (Figure $19, A)$, which can be regarded as adducts of the Lewis acidic and aromatic $B_{3}$ cation with dinitrogen and carbon monoxide. ${ }^{27}$ There too, the aromatic $\mathrm{B}_{3}$ cycle provides electron density for the $\pi$-backbonding interaction, which is necessary to achieve an observable lifetime of the complexes. In a similar manner, OCBBCO (36) is another $\mathrm{CO}$ adduct of boron that has been identified in matrix isolation, and that also features B-to-CO backbonding, ${ }^{228}$ in this case via delocalization of the electron density of the B-B triple bond into the $\pi^{*}$ orbital of the CO ligand.

\subsection{Boron(I) systems}

As detailed above, boron in its favored oxidation state $(+3)$ does not formally possess simultaneously empty and filled orbitals. In its +1 oxidation state, however, it is conceptually expected to possess a nonbonding electron pair in addition to its two empty p-orbitals. For this reason, molecules that possess a boron(I) center in a coordinatively unsaturated environment are excellent candidates for transition-metal-like reactivity (Figure 20). Compounds known as borylenes feature a boron atom that is formally in the +1 oxidation state and are analogues of the more common carbenes. While free monocoordinate borylenes ([:B-R]) are highly reactive intermediates and have not been isolated thus far, the chemistry of their derivatives is a growing field that continues to uncover new and surprising reactivity. In most cases, the borylene fragment is stabilized by one or two Lewis bases or by coordination to a transition metal center. Using this strategy, Bertrand and coworkers were able to isolate the first isolable metal-free borylene $\left[\left({ }^{\mathrm{Cy}} \mathrm{CAAC}\right)_{2} \mathrm{BH}\right] .{ }^{229}$ While free and singly base-stabilized borylenes can be described as metallomimetics, possessing a combination of empty and filled orbitals, tricoordinate borylenes $\left(R B L_{2}\right)$ are coordinatively saturated and their transition metal-like characteristics are thus diminished. Similarly, transition-metal-bound borylenes cannot be considered metallomimetics as they already contain one or more transition metal atoms, which contributes to their reactivity. For these reasons, this chapter will focus on the chemistry of free mono- and dicoordinate borylenes. The other two classes of borylenes have recently been authoritatively reviewed and continue to attract considerable interest. ${ }^{230-236}$
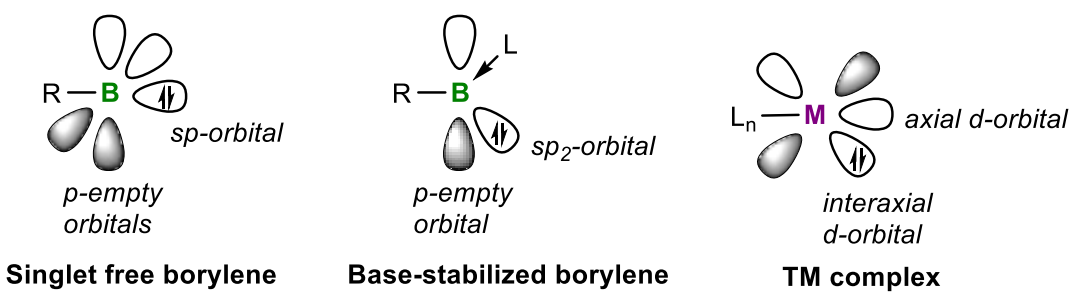

Figure 20. Orbitals relevant to substrate activation in both borylenes and TM complexes.

\subsubsection{Monocoordinate borylenes}

While they remain elusive to isolation and to many methods of solution- and solid-state characterization, the properties and reactivity of monovalent borylenes $([: B-R])$ can be inferred 
from extensive spectroscopic, and theoretical studies as well as from trapping experiments. In all studied cases, the ground state of such molecules is found to be singlet, ${ }^{237,238}$ thus featuring two empty orbitals (LUMO and LUMO+1), as well as a nonbonding lone pair (HOMO) at the sphybridized boron center. This electronic arrangement draws a parallel between monovalent borylenes and coordinatively unsaturated transition metal complexes and suggests their comparable reactivity. Indeed, the reaction of in-situ-generated borylenes with alkynes, carbon monoxide and dinitrogen, ${ }^{239}$ as well as with $\mathrm{C}-\mathrm{H}$ and $\mathrm{C}-\mathrm{C}$ bonds is definitively reminiscent of that of transition metal complexes.

As described earlier, alkynes and olefins readily interact with both the empty and filled orbital of many transition metal centers, forming side-on complexes that are well described by the DewarChatt-Duncanson model (Figure 1)..$^{8,9}$ We have also discussed the formation of metallacyclohexadienes from the reaction of TM complexes with alkynes. ${ }^{214}$ It is this second binding motif which was found by Timms to be dominant in the reactivity of fluoroborylene [FB:] 37 - the first reported transient borylene - and its derivative chloroborylene [CIB:] with acetylenes. ${ }^{240,241}$ Indeed, when generated in the gas phase and exposed to acetylene, both of these hypovalent boron halides afforded, among other products, cyclic $\mathrm{C}_{4} \mathrm{H}_{4} \mathrm{~B}_{2} \mathrm{X}_{2}$ compounds (Figure 21, A). While later computational studies suggested the possibility of these compounds being produced by the dimerization of the corresponding haloborirenes ${ }^{242}$ in a reaction that has precedence in TM chemistry, ${ }^{214}$ the reaction conditions were not conducive to the observation of these presumably short-lived intermediates. Interestingly, 17 years after Timms' report, Pachaly and West $^{243}$ observed the formation of silylborirene $\mathbf{3 8}$ from the low-temperature photolysis of $\mathrm{B}\left(\mathrm{SiPh}_{3}\right)_{3}$ in an organic glass upon warming in the presence of bis(trimethylsilyl)acetylene. While no spectroscopic evidence was obtained for the generation of a borylene species, the authors ascribe the formation of 1-triphenylsilyl-2,3-bis(trimethylsilyl)borirene to the trapping of a free [( $\left.\left.\mathrm{Ph}_{3} \mathrm{Si}\right) \mathrm{B}:\right] 39$ by the acetylene reagent (Figure 21, B). This claim is supported by the characterization of $\mathrm{C}-\mathrm{H}$ and $\mathrm{C}-\mathrm{O}$ activation products when the postulated borylene is generated in the presence of alkane or ether products. 


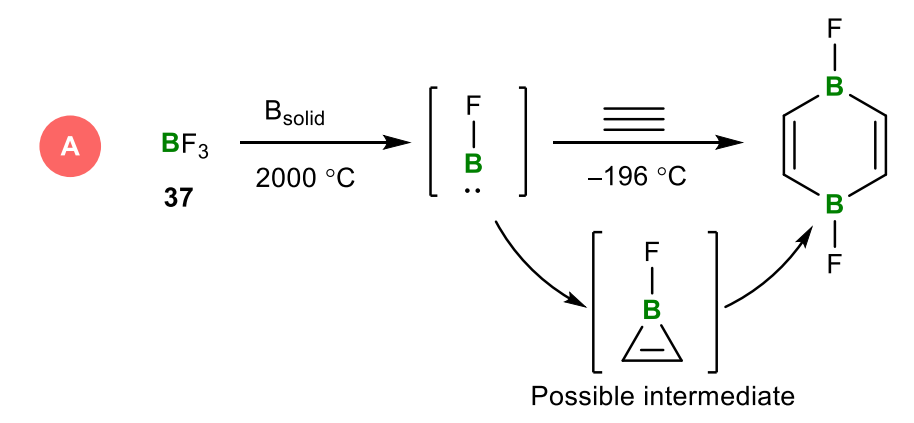

B

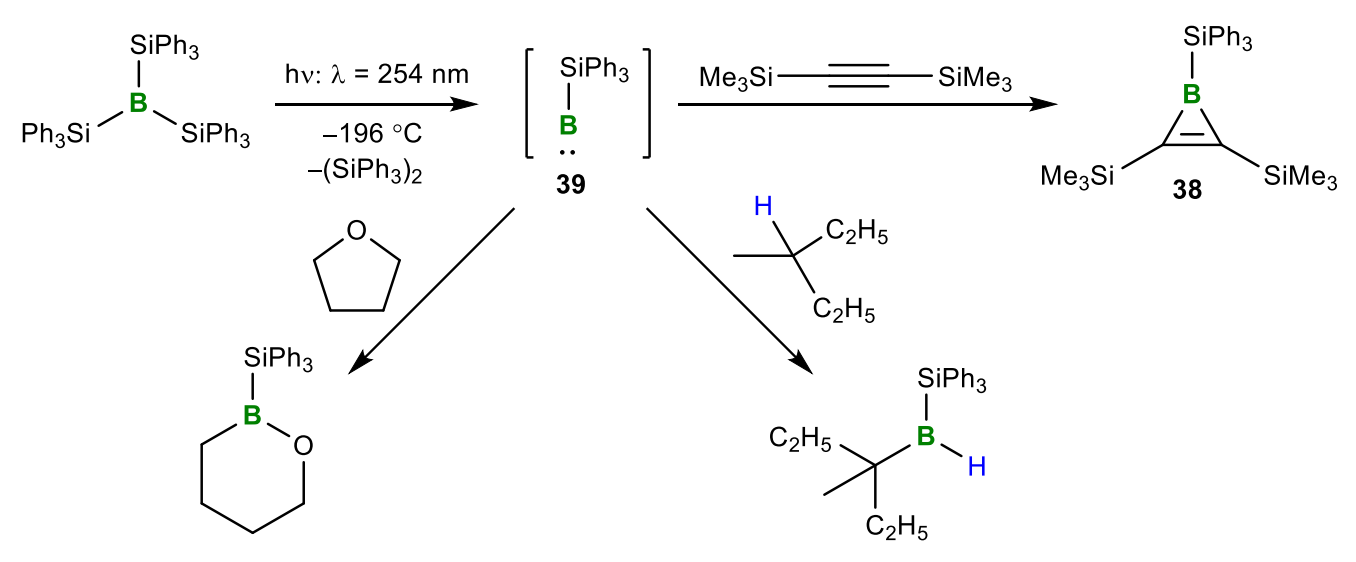

40

Figure 21. Generation and trapping reactions of free fluoro- and silylborylenes.

In the context of the formation of borirene products from the direct addition of a free singlet borylene to an alkyne, a comparison with the side-on coordination of acetylenes with TM is almost unavoidable (Figure 22). In such complexes, the coordination of an alkyne to the singlet borylene pushes the Dewar-Chatt-Duncanson model to the limit of strong alkyne-to-boron donation and boron-to-alkyne backdonation into a rehybridized three-membered ring featuring an elongated C-C bond. ${ }^{244-247}$ This fact illustrates the nature of these highly reactive transient hypovalent boron metallomimetics: while possessing a reactivity that recalls that of TM complexes, their lack of oxidation state flexibility often puts them at the extremes of bonding models. Interestingly, borirenes are also products of the formal transfer of borylene fragments from many transition-metal-stabilized borylenes to alkynes. ${ }^{248-250}$
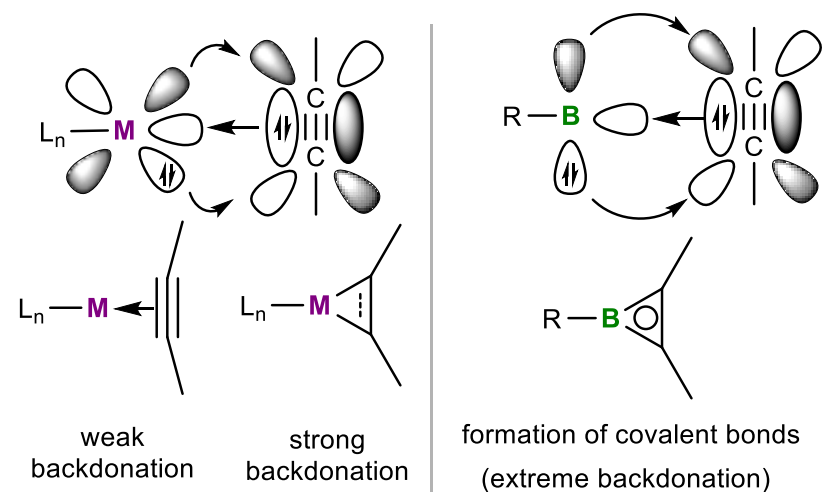

formation of covalent bonds (extreme backdonation) 
Figure 22. Alkyne binding in both TM complexes (left) and borylenes (right).

As described above, the reaction of the putative borylene $\left[\left(\mathrm{Ph}_{3} \mathrm{Si}\right) \mathrm{B}:\right]$ with tert-butane gives 3methylpentyl(triphenylsilyl)borane as $\mathrm{C}-\mathrm{H}$ insertion product 40 . This reaction is also metallomimetic in character and plausibly arises from the interaction of the LUMO of the borylene with the $\mathrm{C}-\mathrm{H} \sigma$-bond of the alkane, with simultaneous electron donation from boron to the $\mathrm{C}-\mathrm{H}$ antibonding orbitals (Figure 23). Such a mechanism would be similar to that of $\mathrm{C}-\mathrm{H}$ bond activation effected by transition metal complexes. ${ }^{251,252}$
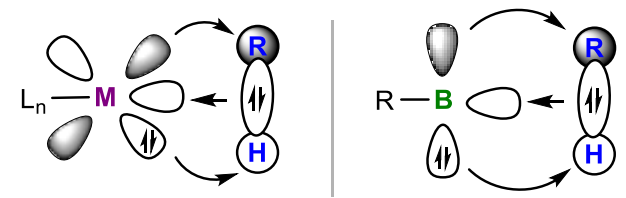

Figure 23. Orbitals relevant to $\mathrm{C}-\mathrm{H}$ activation for TM complexes (left) and borylenes (right).

Similarly to this reactivity, Power and coworkers ${ }^{253}$ observed various insertion reactions when reducing sterically encumbered aryldihaloboranes using different alkali metal reductants. In such reduction reactions the initial formation of a free arylborylene was postulated, followed by its intramolecular insertion into C-C bonds of the aryl substituent (Figure 24, A). The extreme reactivity of free borylenes was even more strikingly demonstrated by Meller and coworkers ${ }^{254,255}$ when they demonstrate that the borylenes generated from the reduction of $\mathrm{X}_{2} \mathrm{BNiPr}_{2}(\mathrm{X}=\mathrm{F}, \mathrm{Cl})$ react even with toluene and other arenes to give various ring-expansion products (Figure 24, B).
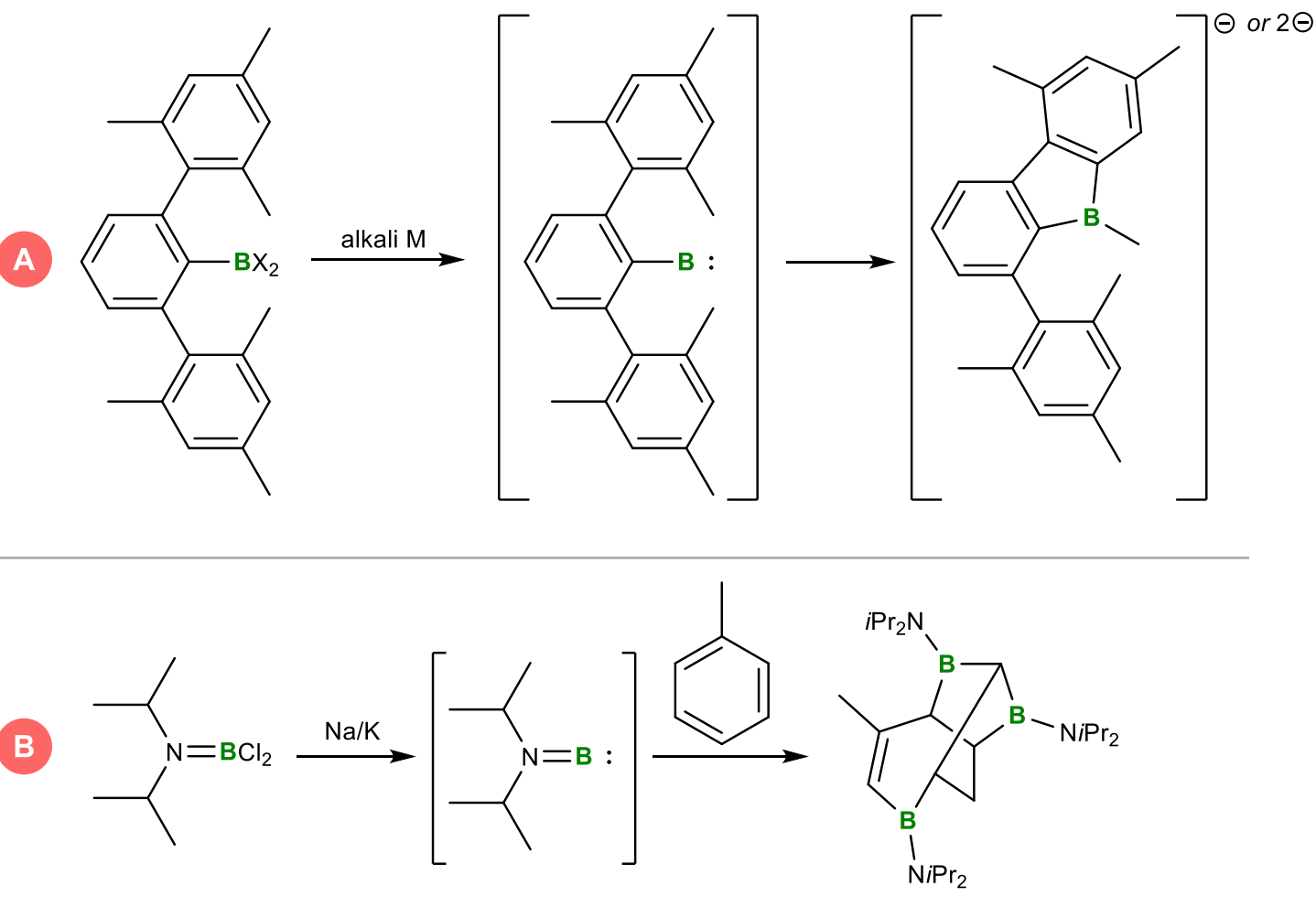
Figure 24. Generation and trapping reactions of free aryl- and aminoborylenes.

Tokitoh and coworkers ${ }^{256}$ have also developed a protocol for the photolytic generation of a bulky arylborylene from $\left[\mathrm{TbtB}(\mathrm{SeMe})_{2}\right](\mathrm{Tbt}=2,4,6$-tris[bis(trimethylsilyl)methyl]phenyl). The resulting [TbtB:] borylene intermediate was trapped by various organochalcogen substrates.

More recently, Bettinger and coworkers ${ }^{257}$ were able to generate and characterize phenylborylene [PhB:] (41) under matrix isolation conditions (Figure 25), via the photolysis of $\mathrm{PhB}\left(\mathrm{N}_{3}\right)_{2}$. Interestingly, generating phenylborylene in $\mathrm{N}_{2}$ - or $\mathrm{CO}$-containing matrices led to the identification of various products, including adducts $[\mathrm{PhB} \leftarrow L]$ of these ligands $\left(L=C O, N_{2}\right)$, which could be characterized on the basis of their IR spectra. Computational studies on these molecules showed that, in close analogy to TM chemistry, B-to-L backdonation is an important component of the bonding in these adducts.

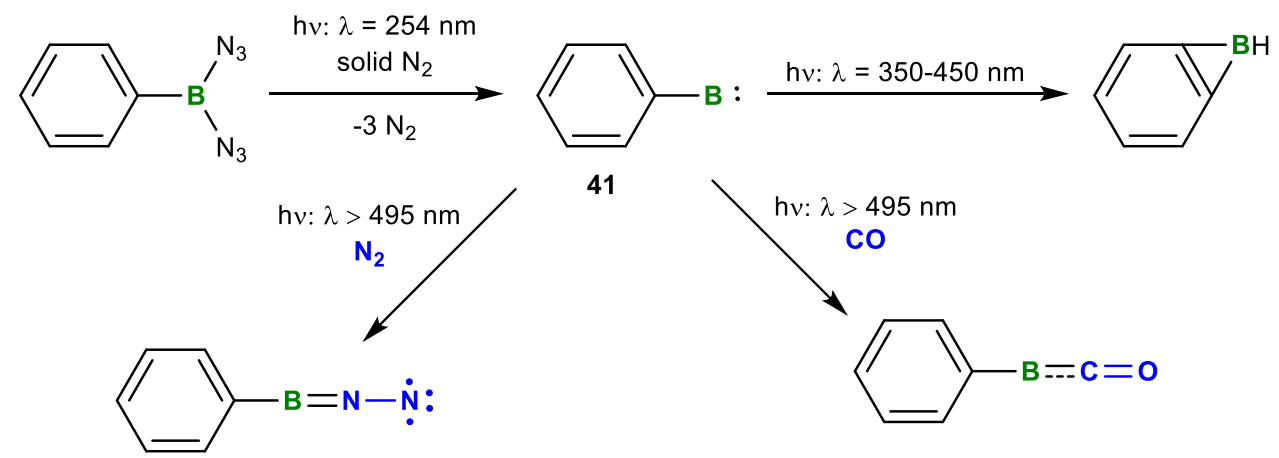

Figure 25. Generation and photolytic reactions of phenylborylene.

\subsubsection{Base-stabilized borylenes}

As the high reactivity of free borylenes significantly hampers their study in the condensed phase, the past few decades have seen an effort to produce and study base-stabilized borylene and borylene-like molecules. Using a similar alkali metal reduction protocol, in 2007 Robinson and coworkers reduced [IDippBBr 3 ] with $\mathrm{KC}_{8}{ }^{194}$ This reaction allowed the authors to isolate diborene product $\mathbf{4 2}$, which is conceptually the dimerization product of the borylene [IDippBH] (Figure 26, A). Following this initial attempt, several reports have described the reduction of NHC-dihaloborane reagents. Many of these have demonstrated the formal insertion of a dicoordinate borylene fragment into intramolecular $\mathrm{C}-\mathrm{H}$ bonds of the $\mathrm{NHC}$ ligand, similarly to free borylenes (Figure 26, B-D). ${ }^{258-260}$ 
A

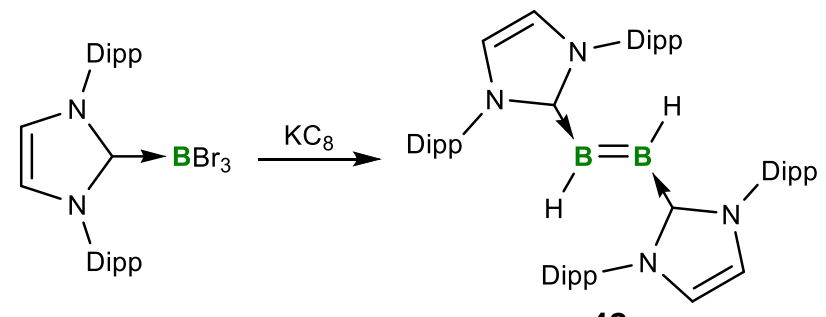

42

B

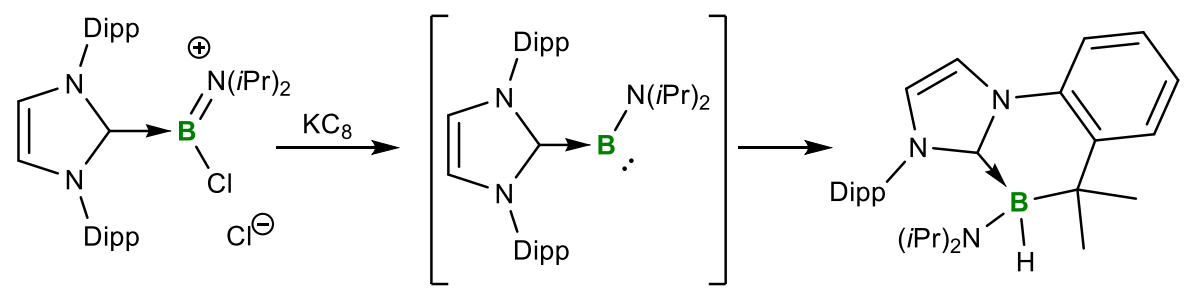

C

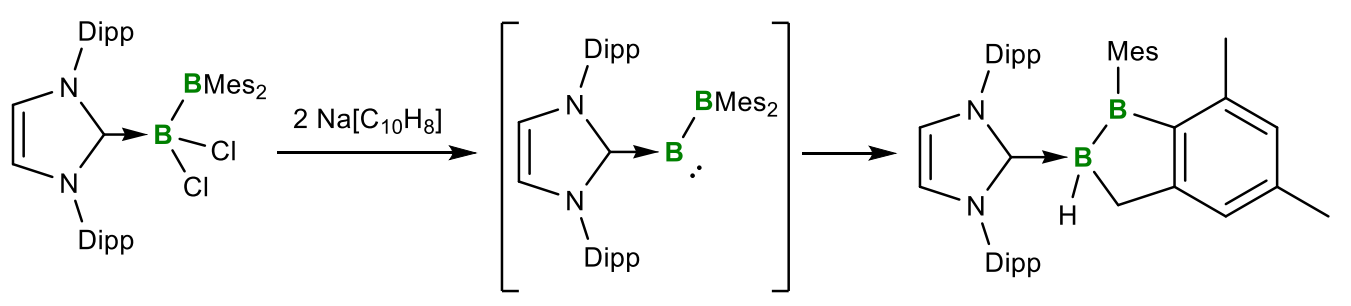

D)<smiles>CC(C)(C)c1nnn([Pb])c1B(Br)Br</smiles><smiles>CCCCCCC(C)C</smiles><smiles>[2H][B-]1(O)c2cccc(C(C)C)c2-n2nnc(C(C)C)c21</smiles><smiles>CC(C)c1cccc2c1[B-](Br)(C(C)C)c1c(C(C)C)nnn1-2</smiles>

Figure 26. Generation and insertion of various NHC-stabilized borylenes.

Interestingly, our group has observed the formation of a base-stabilized borirane (45) from the reduction of $\left[(\mathrm{IMe}) \cdot \mathrm{BHCl}_{2}\right] \quad(\mathrm{IMe}=1,3$-dimethylimidazol-2-ylidene) (43) with sodium naphthalenide. ${ }^{261}$ While the formation of the parent borylene [(IMe) $\left.\mathrm{BH}\right](44)$ and its trapping with naphthalene was invoked to explain the reactivity, the actual mechanism of the transformation is a subject of debate (Figure $27, \mathrm{~A}){ }^{262}$ In a related reaction, our group also observed the formation of the remarkably robust borirane [IMe ${ }^{\mathrm{Me}} \cdot \mathrm{BPh}\left(\mathrm{C}_{14} \mathrm{H}_{12}\right)$ ] (47) when reducing [ $\left.\mathrm{IMe}^{\mathrm{Me}} \cdot \mathrm{BPhCl}_{2}\right](46)$ with $\mathrm{Na}_{2}\left[\mathrm{C}_{14} \mathrm{H}_{12}\right]\left(\mathrm{C}_{14} \mathrm{H}_{12}=1\right.$,2-diphenylethylene) (Figure 27, B). ${ }^{263}$ However, it should be noted that the generation of a borylene was not invoked in the latter case as a nucleophilic substitution mechanism using $\mathrm{Na}_{2}\left[\mathrm{C}_{14} \mathrm{H}_{12}\right]$ is also reasonable. 
A

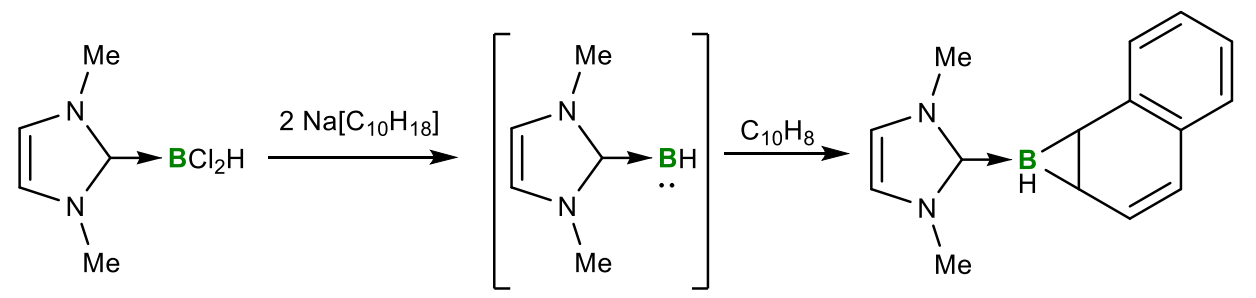

43

44

45

B

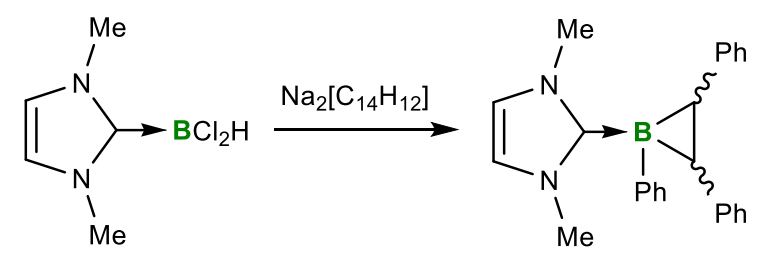

46

47

Figure 27. Trapping of free NHC-borylenes with unsaturated bonds.

In contrast to the previously described examples of highly reactive borylene metallomimetics, a breakthrough in the field was achieved in 2014 by Bertrand, Stephan and coworkers in the isolation of a compound of dicoordinate boron that exhibited a distinct borylene character. ${ }^{264}$ Similar to the generation of highly reactive borylenes, the complex in question was obtained by the reduction - in this case with [ $\left(\mathrm{Me}_{5} \mathrm{C}_{5}\right)_{2} \mathrm{Co}$ ] - of a [ ${ }^{\mathrm{Cy}} \mathrm{CAAC} \cdot \mathrm{BN}\left(\mathrm{SiMe}_{3}\right)_{2} \mathrm{Cl}_{2}$ ] adduct. The product of this reaction, [ $\left.{ }^{\mathrm{Cy}} \mathrm{CAAC} \cdot \mathrm{BN}\left(\mathrm{SiMe}_{3}\right)_{2}\right](48)$, could be seen as the ${ }^{\mathrm{Cy}} \mathrm{CAAC}$ adduct of the borylene [:BN( $\left.\left(\mathrm{SiMe}_{3}\right)_{2}\right]$ and be expected to bear an empty and a filled orbital at the boron atom (Figure 28, A). However, nitrogen-to-boron $\pi$-donation, as well as boron-to-carbene $\pi-$ backdonation stabilize these orbitals and the molecule as a whole. In fact, based on structural analysis of 48 , this compound seems to adopt an allene-type structure in the ground state.
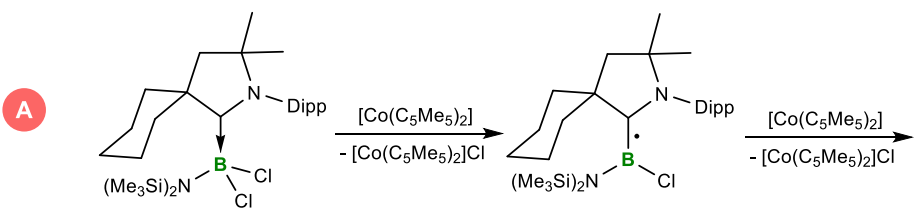
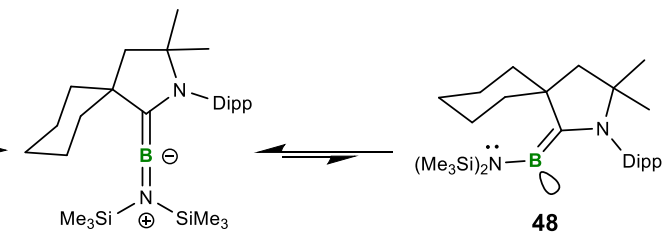

48

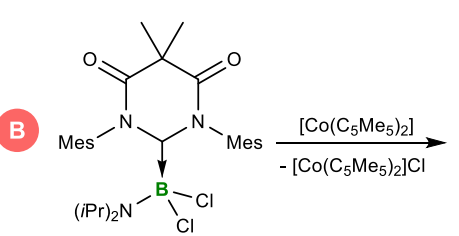<smiles>CCCN(CC)C(=O)N1C(=O)N(C)C(=O)C(C)(C)C1=O</smiles>

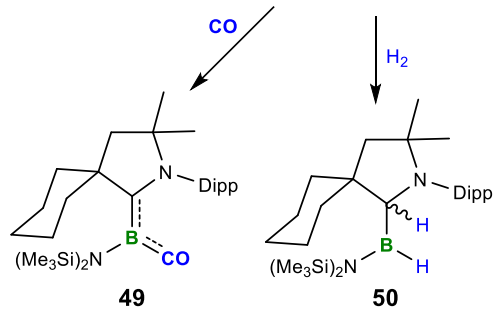

Figure 28. Synthesis and reactivity of isolable NHC-aminoborylenes.

Computational analysis, however, revealed that the borylene character of $\mathbf{4 8}$ was but lightly masked by this donor-acceptor stabilization: bending the molecule at the boron atom to $155^{\circ}$, 
effectively breaking the allenic system, had a small energetic cost of only $5.7 \mathrm{kcal}_{\mathrm{mol}}{ }^{-1}$ and should be feasible thermally. In this bent form, computations showed that boron possesses a low-lying $\mathrm{sp}^{2}$-hybridized LUMO, while the HOMO is still shared with the m-accepting ${ }^{\mathrm{Cy} C A A C}$ ligand and is of $\pi$ symmetry and antibonding with regard to the ${ }^{C y}$ CAAC C-N bond (Figure 29). 48 in its bent form thus possesses a strongly electrophilic boron center which nevertheless also bears a large proportion of the HOMO. Transition-metal-like chemistry should thus be unsurprising with such a complex.
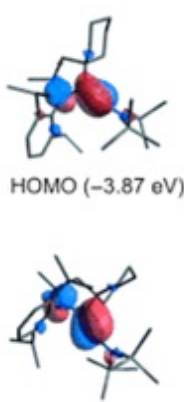

$\operatorname{HOMO}(-3.82 \mathrm{eV})$
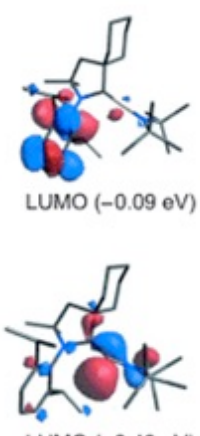

LUMO $(-0.48 \mathrm{eV})$
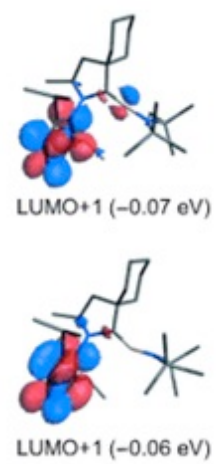
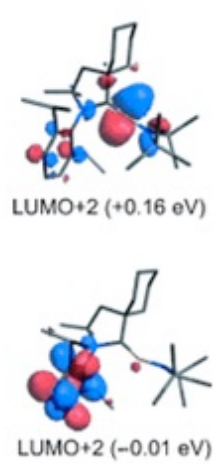

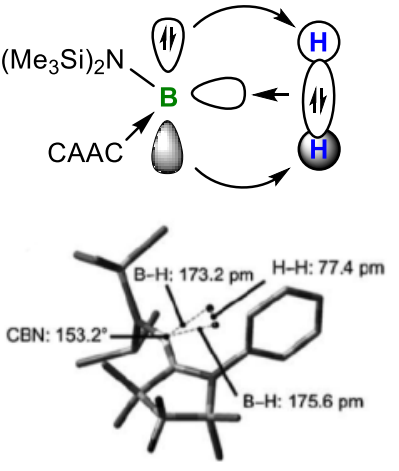

Figure 29. Orbitals in $\mathbf{4 8}$ in the ground state (top left) and in a constrained $155^{\circ}$ bent geometry (bottom left) relevant to its reaction with $\mathrm{H}_{2}$. Representation of the transition state of $\mathrm{H}_{2}$ cleavage by $\mathbf{4 8}$ to give $\mathbf{5 0}$ (right).

Indeed, $\mathbf{4 8}$ was shown to react with carbon monoxide and form a stable coordination complex (Figure 28, A), a reaction that is exceedingly rare for boron complexes. Infrared spectroscopy $\left(v_{\mathrm{CO}}=1956 \mathrm{~cm}^{-1}\right)$ as well as computational modelling revealed that boron-to-CO $\pi$-backbonding is a significant aspect of the coordination of carbon monoxide and that the $\pi$ symmetrical $\mathrm{HOMO}$ is shared between the ${ }^{\mathrm{C}} \mathrm{CAAC}$ and the $\mathrm{CO}$ ligands. Consistent with this relocation of the $\mathrm{HOMO}$, the solid-state $\mathrm{B}^{-}{ }^{\mathrm{Cy}} \mathrm{CAAC}$ bond distance increases from 48 (1.401(5) $\AA$ ) to 49 (1.505(4) A). 48 can also cleave dihydrogen in a mechanism that is decidedly metallomimetic: according to computational studies, the most likely mechanism involves the interaction of the $\mathrm{H}-\mathrm{H} \sigma$-bond with the boron-centered LUMO of the bent 48 , while backdonation into the $\mathrm{H}-\mathrm{H} \sigma^{*}$ orbital completes the bond cleavage. These results clearly show that the metallomimetic reactivity of borylene compounds can be harnessed into selective intermolecular reactions, and that donoracceptor stabilization of borylenes does not completely mask their TM-like characteristics. A similarly stabilized compound was reported by Hudnall and Ledet in $2016,{ }^{265}$ using a $\pi-$ accepting N,N'-diamidocarbene (DAC), ${ }^{266}$ although as yet no reactivity of the compound has been reported (Figure 28, B).

It is pertinent to mention here the doubly silylene-stabilized borylene cation $51^{267,268}$ which was prepared by Xie and coworkers through the substitution of a bromide from a bis-silylene bromoborylene with $\mathrm{CO}$ from $\left[\mathrm{W}(\mathrm{CO})_{6}\right]$ (Figure 30). While this reaction does not involve the formal binding of free carbon monoxide by a boron center, the product is one of the few examples of a stable B-CO adduct. Structural, computational and spectroscopic evidence shows the presence of B-to-CO backbonding, which is typically a TM-CO characteristic. An IR 
CO stretching band at $2021 \mathrm{~cm}^{-1}$ and a C-O bond distance of 1.046(6) $\AA$ indicates, however, the presence of a lower degree of backbonding than in 49 , which can be expected from the cationic nature of the complex. This $\mathrm{CO}$ complex spontaneously loses one equivalent of carbon monoxide in the presence of molecular hydrogen at $80{ }^{\circ} \mathrm{C}$, leading to the TM-like cleavage of $\mathrm{H}_{2}$ at the boron center and to complex $\mathbf{5 2}$.

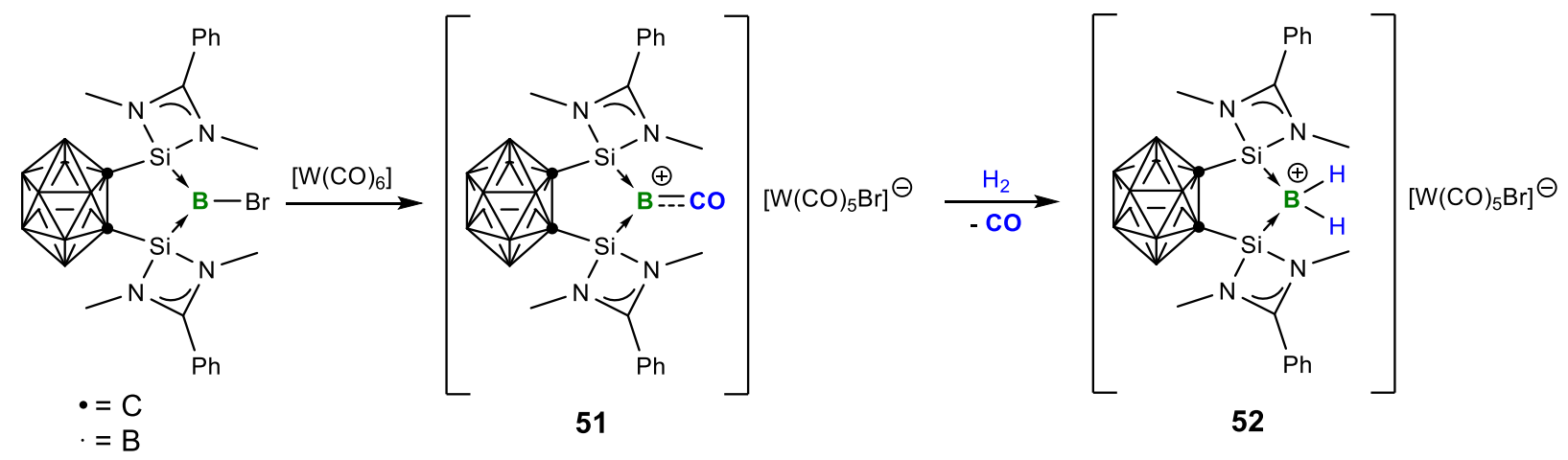

Figure 30. $\mathrm{CO}$ extrusion and $\mathrm{H}_{2}$ uptake by a cationic doubly silylene-stabilized borylene.

For our part, we have endeavored to uncover metallomimetic reactivity with a dicoordinate borylene that features a smaller degree of donor-acceptor stabilization. We reasoned that generating borylene compounds that possess an inert aryl group (in contrast to the m-donating amino group of Bertrand and Stephan's allene) would be an important step in that direction. Previous work from our group found that the reduction of $\left[{ }^{\mathrm{Me}} \mathrm{CAAC} \cdot \mathrm{BDurCl}_{2}\right]$ (Dur = 2,3,5,6tetramethylphenyl) by decamethylcobaltocene or $\mathrm{KC}_{8}$ afforded the remarkably stable boron radical $\left[\left({ }^{\mathrm{Me}} \mathrm{CAAC}\right) \mathrm{BCIDur}\right]{ }^{269}$ Unfortunately, further reduction to a borylene species was not achieved. For this reason, we developed an alternative borylene generation method: the transfer of a borylene fragment from metal-carbonyl complexes to suitable Lewis bases can afford $\mathrm{CO}$ complexes of metal-free borylenes. This approach was first found to be compatible with borylene transfer from [(OC) $\left.{ }_{5} \mathrm{Mn}=\mathrm{B}-\mathrm{Tp}\right] 53$ ( $\mathrm{Tp}=$ 2,6-bis(triisopropylphenyl)phenyl) to additional carbon monoxide or to :CNDipp (Dipp $=2,6$-diisopropylphenyl), yielding the tricoordinate borylenes [(OC) $\left.)_{2} \mathrm{BTp}\right]($ 54-CO) and [(DippNC)(OC)BTp] (54-CNDipp), respectively (Figure 31). ${ }^{270}$ These complexes showed strong B-to-CO backdonation, both from their structural ( $d_{\mathrm{C}-\mathrm{O}} 1.152(2) \AA$ and $1.159(2) \AA$, respectively) and spectroscopic characterization (IR $\mathrm{V}_{\mathrm{CO}}=1942$ and $1930 \mathrm{~cm}^{-1}$ respectively). Apart from adducts of carbon and nitrogen, 54-CO remains the only example of multiple coordination of carbon monoxide at a p-block element, being thus comparable to poly-CO TM complexes.

For its part, 54-CNDipp proved to undergo CO extrusion under UV irradiation, presumably generating a transient free dicoordinate borylene [DippNCBTp] (Figure 31). Although the photolytic formation of a dicoordinate borylene $\mathbf{5 5}$ could not be ascertained spectroscopically, a $\mathrm{C}-\mathrm{C}$ insertion product (56) was isolated from the reaction, which is consistent with the transient formation of [DippNCBTp]. 

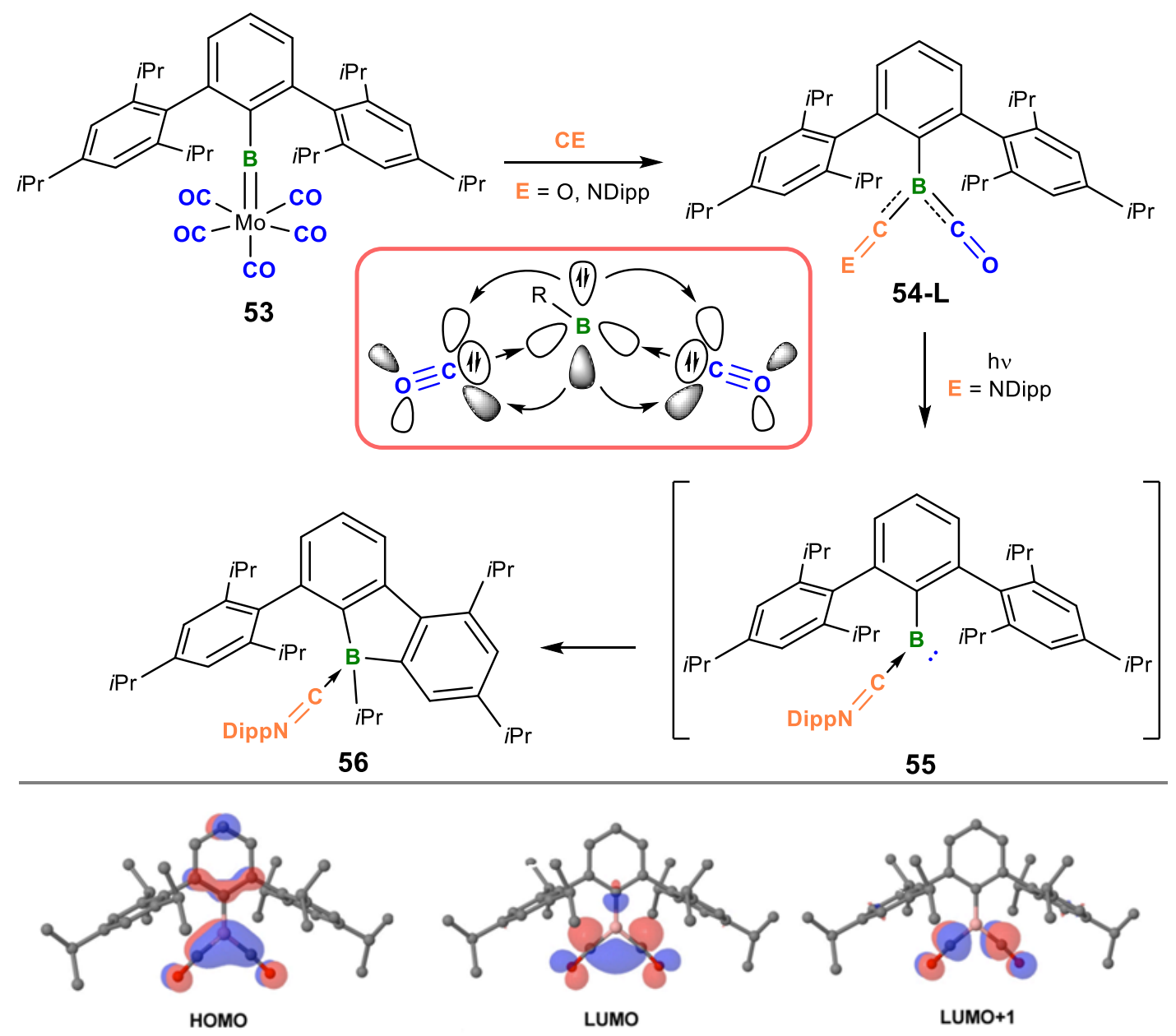

Figure 31. The generation of carbonyl-borylene 54-L, photolytic reactions (Top). Frontier molecular orbitals of of bis-carbonyl borylene 54-CO. 


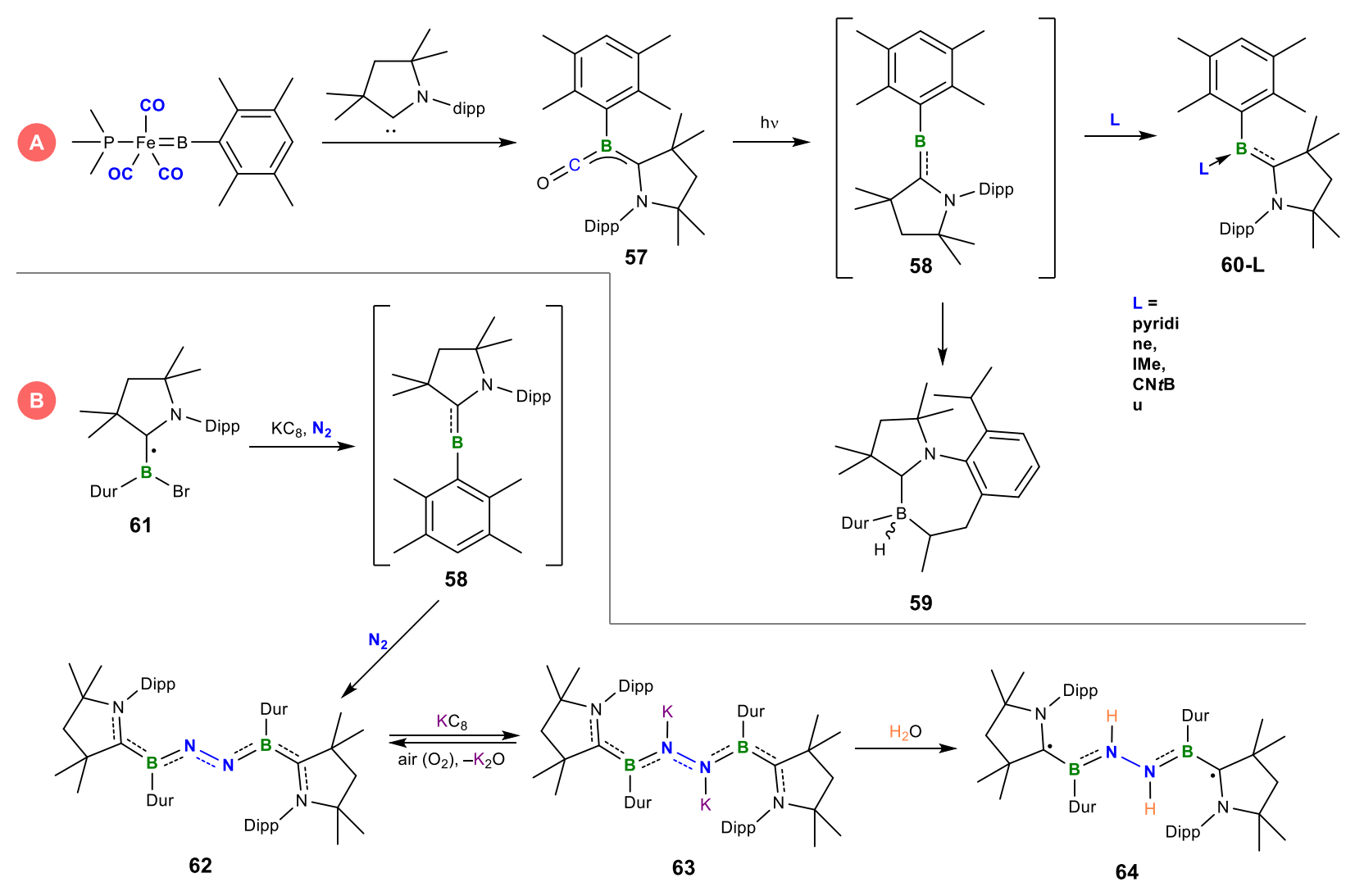

Figure 32. The generation of free borylenes via photolytic $\mathrm{CO}$ extrusion and their capture and insertion reactions ( $A$ and $B$ ). Capture, reduction and protonation of $\mathrm{N}_{2}$ at a borylene center (C).

Similarly, borylene transfer was also developed from $\left[\left(\mathrm{Me}_{3} \mathrm{P}\right)(\mathrm{OC})_{3} \mathrm{Fe}=\mathrm{BDur}\right]^{271}$ using ${ }^{\mathrm{Me}} \mathrm{CAAC}$ as a $\pi$-accepting base to afford [( $\left.\left.{ }^{\mathrm{Me}} \mathrm{CAAC}\right)(\mathrm{OC}) \mathrm{BDur}\right](\mathbf{5 7})$ (Figure 31, B). ${ }^{4} \mathbf{5 7}$ is an analog of $\mathbf{4 9}$, but features a substantially shorter B-CO bond (1.469(2) $\AA$ ), suggesting a larger amount of boron-to-CO backdonation, which is further supported by an IR $v_{C O}$ stretch of $1942 \mathrm{~cm}^{-1}$. Interestingly, $\mathbf{5 7}$ also loses an equivalent of carbon monoxide upon UV irradiation, affording the intramolecular insertion product $\mathbf{5 9}$ at ambient temperature. In this case, however, the insertion takes place into a remote $\mathrm{C}-\mathrm{H}$ bond of the CAAC ligand, forming a seven-membered ring that would intuitively be thought to be disfavored. The transient formation of an electrophilic dicoordinate borylene (58) could be proven in this case by trapping experiments with various Lewis bases to give a family of new doubly base-stabilized borylene complexes (60-L) (Figure 32, A). ${ }^{4}$ This photolytic extrusion of carbon monoxide is another metallomimetic characteristic of borylenes. Indeed, the photodissociation of carbon monoxide is a standard reactivity pattern of transition metal carbonyl complexes.

The relatively long lifetime of the dicoordinate borylene $\mathbf{5 8}$ in this system - allowing it to be trapped by stoichiometric reagents - prompted us to explore its reactivity with other substrates which are usually unreactive with nonmetal reagents. In particular, we hypothesized that dinitrogen, being a less reactive $\mathrm{CO}$ analog, could be fixed by such a reactive borylene. Initial attemps to photolyze $\mathbf{5 7}$ in the presence of $\mathrm{N}_{2}$ did not yield any nitrogen fixation products. For this reason, we revisited approaches for the generation of free 58. Thus, we found that the 
reduction of [ ${ }^{\mathrm{Me}} \mathrm{CAAC} \cdot \mathrm{BDurBr}_{2}$ ] using $\mathrm{KC}_{8}$ did produce transient $\mathbf{5 8}$ and that the borylene could be trapped, even using $\mathrm{CO}$ as a gaseous reagent (to yield 57). Through a similar reduction protocol $\left(\mathrm{KC}_{8}\right.$, toluene) under an atmosphere of $\mathrm{N}_{2}$, we were able to isolate, albeit in low yield, [\{ $(\mathrm{CAAC})$ DurB $\left.\left.]_{2}\left(\mu^{2}-\mathrm{N}_{2}\right)\right\}\right]$ (62) as a bridging end-on complex of dinitrogen (Figure 32, B). ${ }^{272}$ By optimizing the reaction conditions - using the [ ${ }^{\mathrm{Me}} \mathrm{CAAC} \cdot \mathrm{BDurBr}$ ] radical (61) as a starting reagent and an excess of $\mathrm{KC}_{8}$ and up to 4 atm. of dinitrogen - we were able to increase the yield of the reaction to provide $64 \%$ of $\left\{[(C A A C) D u r B]_{2}\left(\mu^{2}-N_{2} K_{2}\right)\right\}(63)$, an overreduction product of dinitrogen complex 62 . This latter species can be selectively oxidized back to the neutral complex 62 using ambient air.

The bonding situation in these species is understandably complicated, but is once again reminiscent of transition metal chemistry, in which the end-on bridging binding mode is common for $\mathrm{N}_{2}$. In analogy with the complexation of $\mathrm{CO}$ to 58 , the B-N bonds in 62 involve a B-to- $\mathrm{N}$ mbackdonation that is shared with the B-to-CAAC m-interaction. In fact, in 62, the B-CAAC bonds (1.528(5) $\AA$ and 1.541(4) $\AA$ ) are longer than in 57 (1.499(2) $\AA$ ), suggesting that the bridging $N_{2}$ ligand accepts a larger degree of backdonation than $\mathrm{CO}$. While the end-on bridging dinitrogen ligand in TM complexes is generally linear, the bent form it adopts in compound 62 can be explained by the geometry of the orbitals involved in the backdonation: while TMs can involve up to two orthogonal $d$-orbitals in backbonding, the borylene has a single available filled orbital of $\pi$-symmetry (Figure 33). ${ }^{273,274}$ Nevertheless, the degree of activation of dinitrogen by the borylene - as suggested by the length of the $\mathrm{N}-\mathrm{N}$ bond that is weakened by backbonding $(1.248(4) \AA)$ - is superior than in many TM complexes. ${ }^{273}$
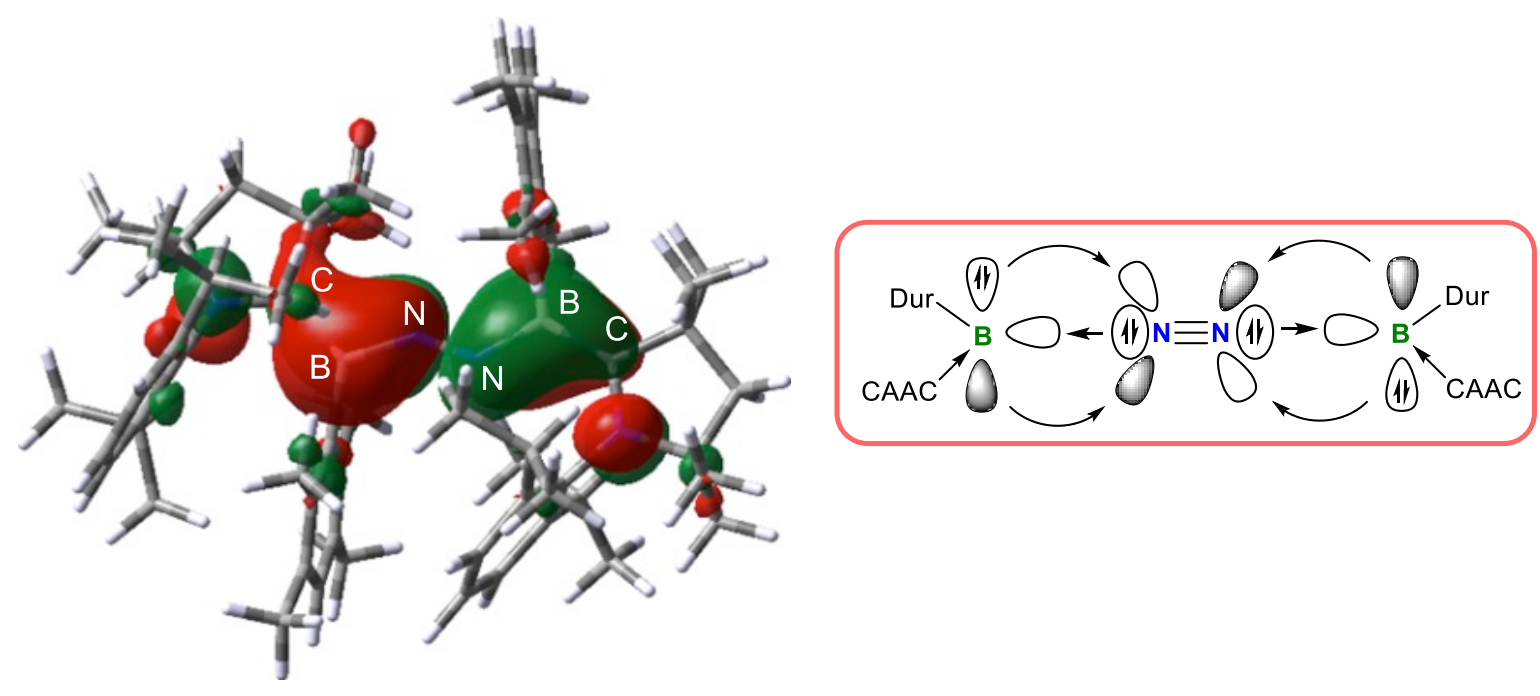

Figure 33. Calculated HOMO-1 of 62 (left) at the M062X/6-311g(d,p) level and schematic representation of the backbonding (right).

The reduction of metal-bound dinitrogen by potassium is an established reaction. ${ }^{275}$ Similarly, as mentioned above, 62 can be reduced by $\mathrm{KC}_{8}$ to give the dipotassium complex 63 . Similarly to iron complexes reported by Holland and coworkers, this reduction is accompanied by a 
lengthening of the $\mathrm{N}-\mathrm{N}$ bond by ca. $4.5 \%$ (to $1.304(3) \AA$ ). The fact that the dinitrogen ligand is reduced is also reflected in the $B-N$ and $B-{ }^{M e} C A A C$ bond distances, which suggest a diminished B-to-N backdonation.

The reduced 63 species can be selectively protonated by water to give $\left\{[(\mathrm{CAAC}) \mathrm{DurB}]_{2}\left(\mu^{2}-\right.\right.$ $\left.\left.\mathrm{N}_{2} \mathrm{H}_{2}\right)\right\}$ 64. This new complex features a very long $\mathrm{N}-\mathrm{N}$ bond $(1.402(2) \AA$ ) that is in the range of single bonds. Furthermore, the triplet diradical nature of 64 reveals that, in contrast to 62 and 63, full covalent bonds exist between the $\mathrm{N}_{2} \mathrm{H}_{2}$ ligand and the boron centers. Complex 64 is thus considered a diborahydrazine complex, which has been formed directly from dinitrogen from a reduction-protonation sequence. This transformation gives hope towards the further reduction and functionalization of dinitrogen, which is a lingering goal of transition-metal-mediated $\mathrm{N}_{2}$ chemistry.

\subsection{Conclusion}

We have thus seen that a few strategies enable metallomimetic reactivity at a single boron site. While strong Lewis acidity in boranes leads to the activation of dihydrogen and to gas-phase interactions with $\mathrm{CO}$ and $\mathrm{N}_{2}$, a richer reactivity is obtained from species that are capable of $\Pi$ backdonation because of their electronic and orbital arrangement. To this day, most examples of such compounds belong to the borylene family, either as free borylenes or base-stabilized ones. While the former display strong reactivity towards alkynes, $\mathrm{CO}, \mathrm{C}-\mathrm{H}$ and $\mathrm{C}-\mathrm{C}$ bonds, their instability makes them difficult to handle. The advent of base-stabilized borylenes, on the other hand, is starting a trend of isolable or long-lived boron metallomimetics. The binding and activation of $\mathrm{CO}, \mathrm{H}_{2}$ and even $\mathrm{N}_{2}$ by these complexes is only the beginning of the study of these fascinating molecules.

\section{General Summary}

The electropositivity of boron with respect to other light main group elements renders it uniquely reactive. In this review, we have highlighted the three principle methods by which boron can achieve reactivity akin to transition metals: (1) Boron forms stable electron deficient complexes featuring a B-centered low-lying vacant orbital which can react with with inert substrates, including $\mathrm{H}_{2}$ and $\mathrm{C}-\mathrm{H}$ bonds, in combination with an external electron donor. So called 'Frustrated Lewis pairs' are the most mature field of boron metallomimetics, and have been applied to catalytic reactions, most notably to hydrogenation and $\mathrm{C}-\mathrm{H}$ borylation reactions. (2) Systems featuring B-B single and multiple bonds also readily capture and couple small molecules including $\mathrm{CO}$, alkynes, and $\mathrm{H}_{2}$, due to the relatively low HOMO-LUMO gaps within $\mathrm{B}$ $\mathrm{B}$ bonded systems. Furthermore, the reactivity of such species is amplified by electronic transitions that can be readily accessed photolytically. (3) Finally, boron (I) species have began to emerge as truly single-site metallomimetics, and has allowed the capture of $\mathrm{CO}, \mathrm{H}_{2}$ and most recently $\mathrm{N}_{2}$. The accelerating pace of research into this evolving field will doubtlessly continue to yield important discoveries and reactivities reminiscent of transition metal chemistry. The ability of boron to 'mimic' the activation of transition metals points to a greener future where chemistry first established by expensive and toxic transition metals can equivalently accomplished by earth-abundant elements. 


\section{Acknowledgements}

H.B. gratefully acknowledges the Deutsche Forschungsgemeinschaft and the European Research Council (ERC) under the European Union Horizon 2020 Research and Innovation Program (grant agreement no. 669054) for funding. C.P. and M.-A.L. thank the Alexander von Humboldt Foundation and the NSERC for postdoctoral fellowships.

\section{References}

(1) IUPAC: Compendium of Chemical Terminology, 2nd ed. (the "Gold Book"). Blackwell Scientific Publications, Oxford 1997.

(2) Cotton, F. A. W.: Advanced Inorganic Chemistry; Wiley: New York, 1988.

(3) Masters, C.: Homogeneous Transition-metal Catalysis: A Gentle Art; Chapman and Hall: New York,

1980.

(4) Braunschweig, H.; Krummenacher, I.; Légaré, M.-A.; Matler, A.; Radacki, K.; Ye, Q. Main-Group Metallomimetics: Transition Metal-like Photolytic CO Substitution at Boron. J. Am. Chem. Soc. 2017, $139,1802-1805$.

(5) Power, P. P. Main-group elements as transition metals. Nature 2010, 463, 171.

(6) Erker, G.; Stephan, D. W.: Frustrated Lewis Pairs I: Uncovering and Understanding. Erker, G., Stephan, D. W., Eds.; Springer Berlin Heidelberg: Berlin, Heidelberg, 2013.

(7) $\quad$ Erker, G.; Stephan, D. W.: Frustrated Lewis Pairs II: Expanding the Scope. Erker, G., Stephan, D. W., Eds.; Springer Berlin Heidelberg: Berlin, Heidelberg, 2013.

(8) Dewar, M. Bull. Soc. Chim. Fr. 1951, 18, C79.

(9) Chatt, J.; Duncanson, L. A. 586. Olefin co-ordination compounds. Part III. Infra-red spectra and structure: attempted preparation of acetylene complexes. J. Chem. Soc. (Resumed) 1953, 2939-2947.

(10) McCahill, J. S. J.; Welch, G. C.; Stephan, D. W. Reactivity of "Frustrated Lewis Pairs": ThreeComponent Reactions of Phosphines, a Borane, and Olefins. Angew. Chem. Int. Ed. 2007, 46, 4968-4971.

(11) Stephan, D. W. The broadening reach of frustrated Lewis pair chemistry. Science 2016, 354.

(12) Stephan, D. W.; Erker, G. Frustrated Lewis Pairs: Metal-free Hydrogen Activation and More.

Angew. Chem. Int. Ed. 2010, 49, 46-76.

(13) Stephan, D. W.; Erker, G. Frustrated Lewis Pair Chemistry: Development and Perspectives. Angew. Chem. Int. Ed. 2015, 54, 6400-6441.

(14) Stephan, D. W. Frustrated Lewis Pairs. J. Am. Chem. Soc. 2015, 137, 10018-10032.

(15) Fontaine, F.-G.; Stephan, D. W. On the concept of frustrated Lewis pairs. Philosophical Transactions of the Royal Society A: Mathematical, 2017, 375.

(16) Fontaine, F.-G.; Rochette, É. Ambiphilic Molecules: From Organometallic Curiosity to Metal-Free Catalysts. Acc. Chem. Res. 2018, 51, 454-464.

(17) Rokob, T. A.; Bakó, I.; Stirling, A.; Hamza, A.; Pápai, I. Reactivity Models of Hydrogen Activation by Frustrated Lewis Pairs: Synergistic Electron Transfers or Polarization by Electric Field? J. Am. Chem. Soc. 2013, $135,4425-4437$.

(18) Rokob, T. A.; Hamza, A.; Stirling, A.; Soós, T.; Pápai, I. Turning Frustration into Bond Activation: A Theoretical Mechanistic Study on Heterolytic Hydrogen Splitting by Frustrated Lewis Pairs. Angew. Chem. Int. Ed. 2008, 47, 2435-2438.

(19) Parks, D. J.; Piers, W. E. Tris(pentafluorophenyl)boron-Catalyzed Hydrosilation of Aromatic Aldehydes, Ketones, and Esters. J. Am. Chem. Soc. 1996, 118, 9440-9441.

(20) Wittig, G.; Benz, E. Über das Verhalten von Dehydrobenzol gegenüber nucleophilen und elektrophilen Reagenzien. Chem. Ber. 1959, 92, 1999-2013.

(21) Tochtermann, W. Struktur und Reaktionsweise organischer at-Komplexe. Angew. Chem. 1966, 78, 355-375

(22) Welch, G. C.; Juan, R. R. S.; Masuda, J. D.; Stephan, D. W. Reversible, Metal-Free Hydrogen Activation. Science 2006, 314, 1124-1126.

(23) Welch, G. C.; Stephan, D. W. Facile Heterolytic Cleavage of Dihydrogen by Phosphines and Boranes. J. Am. Chem. Soc. 2007, 129, 1880-1881.

(24) Crabtree, R. H. Dihydrogen complexes: some structural and chemical studies. Acc. Chem. Res. $1990,23,95-101$. 
(25) Kubas, G. J. Fundamentals of $\mathrm{H}_{2}$ Binding and Reactivity on Transition Metals Underlying Hydrogenase Function and $\mathrm{H}_{2}$ Production and Storage. Chem. Rev. 2007, 107, 4152-4205.

(26) Berke, H. Conceptual Approach to the Reactivity of Dihydrogen. ChemPhysChem 2010, 11, 1837-

1849.

(27) Clapham, S. E.; Hadzovic, A.; Morris, R. H. Mechanisms of the $\mathrm{H}_{2}$-hydrogenation and transfer hydrogenation of polar bonds catalyzed by ruthenium hydride complexes. Coord. Chem. Rev. 2004, 248, $2201-2237$.

(28) Morris, R. H. Mechanisms of the $\mathrm{H}_{2-}$ and transfer hydrogenation of polar bonds catalyzed by iron group hydrides. Dalton. Trans. 2018.

(29) Liu, L.; Lukose, B.; Jaque, P.; Ensing, B. Reaction Mechanism of Hydrogen Activation by Frustrated Lewis Pairs. Green Energy \& Environment 2018.

(30) Grimme, S.; Kruse, H.; Goerigk, L.; Erker, G. The Mechanism of Dihydrogen Activation by Frustrated Lewis Pairs Revisited. Angew. Chem. Int. Ed. 2010, 49, 1402-1405.

(31) Brown, L. C.; Hogg, J. M.; Gilmore, M.; Moura, L.; Imberti, S.; Gärtner, S.; Gunaratne, H. Q. N.; O’Donnell, R. J.; Artioli, N.; Holbrey, J. D.; Swadźba-Kwaśny, M. Frustrated Lewis pairs in ionic liquids and molecular solvents - a neutron scattering and NMR study of encounter complexes. Chem. Commun. 2018, 54, 8689-8692.

(32) Rokob, T. A.; Hamza, A.; Pápai, I. Rationalizing the Reactivity of Frustrated Lewis Pairs: Thermodynamics of $\mathrm{H}_{2}$ Activation and the Role of Acid-Base Properties. J. Am. Chem. Soc. 2009, 131, 1070110710.

(33) Skara, G.; De Vleeschouwer, F.; Geerlings, P.; De Proft, F.; Pinter, B. Heterolytic Splitting of Molecular Hydrogen by Frustrated and Classical Lewis Pairs: A Unified Reactivity Concept. Sci. Rep. 2017, 7, 16024.

(34) Schirmer, B.; Grimme, S. Electric field induced activation of $\mathrm{H}_{2}$-Can DFT do the job? Chem. Commun. 2010, 46, 7942-7944.

(35) Liu, L.; Lukose, B.; Ensing, B. Hydrogen Activation by Frustrated Lewis Pairs Revisited by Metadynamics Simulations. J. Phys. Chem. C. 2017, 121, 2046-2051.

(36) Geier, S. J.; Gilbert, T. M.; Stephan, D. W. Activation of H2 by Phosphinoboranes $R_{2} P B\left(C_{6} F\right)_{2}$. J. Am. Chem. Soc. 2008, 130, 12632-12633.

(37) Nyhlén, J.; Privalov, T. "Frustration" of Orbital Interactions in Lewis Base/Lewis Acid Adducts: A Computational Study of $\mathrm{H} 2$ Uptake by Phosphanylboranes $\mathrm{R}_{2} \mathrm{P}=\mathrm{BR}_{2}^{\prime}$. Eur. J. Inorg. Chem. 2009, 2009, $2759-2764$.

(38) Dettenrieder, N.; Aramaki, Y.; Wolf, B. M.; Maichle-Mössmer, C.; Zhao, X.; Yamashita, M.; Nozaki, K.; Anwander, R. Assessing the Brønsted Basicity of Diaminoboryl Anions: Reactivity toward Methylated Benzenes and Dihydrogen. Angew. Chem. Int. Ed. 2014, 53, 6259-6262.

(39) Hounjet, L. J.; Stephan, D. W. Hydrogenation by Frustrated Lewis Pairs: Main Group Alternatives to Transition Metal Catalysts? Org. Process Res. Dev. 2014, 18, 385-391.

(40) Chase, P. A.; Jurca, T.; Stephan, D. W. Lewis acid-catalyzed hydrogenation: $B\left(C_{6} F_{5}\right)_{3}$-mediated reduction of imines and nitriles with $\mathrm{H}_{2}$. Chem. Commun. 2008, 1701-1703.

(41) Chase, P. A.; Welch, G. C.; Jurca, T.; Stephan, D. W. Metal-Free Catalytic Hydrogenation. Angew. Chem. Int. Ed. 2007, 46, 8050-8053.

(42) Stephan, D. W.; Greenberg, S.; Graham, T. W.; Chase, P.; Hastie, J. J.; Geier, S. J.; Farrell, J. M.; Brown, C. C.; Heiden, Z. M.; Welch, G. C.; Ullrich, M. Metal-Free Catalytic Hydrogenation of Polar Substrates by Frustrated Lewis Pairs. Inorg. Chem. 2011, 50, 12338-12348.

(43) Spies, P.; Erker, G.; Kehr, G.; Bergander, K.; Fröhlich, R.; Grimme, S.; Stephan, D. W. Rapid intramolecular heterolytic dihydrogen activation by a four-membered heterocyclic phosphane-borane adduct. Chem. Commun. 2007, 5072-5074.

(44) Sumerin, V.; Schulz, F.; Atsumi, M.; Wang, C.; Nieger, M.; Leskelä, M.; Repo, T.; Pyykkö, P.; Rieger, B. Molecular Tweezers for Hydrogen: Synthesis, Characterization, and Reactivity. J. Am. Chem. Soc. 2008, 130, 14117-14119.

(45) Oestreich, M.; Hermeke, J.; Mohr, J. A unified survey of Si-H and H-H bond activation catalysed by electron-deficient boranes. Chem. Soc. Rev. 2015, 44, 2202-2220.

(46) Reddy, J. S.; Xu, B.-H.; Mahdi, T.; Fröhlich, R.; Kehr, G.; Stephan, D. W.; Erker, G. AlkenylboraneDerived Frustrated Lewis Pairs: Metal-Free Catalytic Hydrogenation Reactions of Electron-Deficient Alkenes. Organometallics 2012, 31, 5638-5649.

(47) Mahdi, T.; Stephan, D. W. Enabling Catalytic Ketone Hydrogenation by Frustrated Lewis Pairs. J. Am. Chem. Soc. 2014, 136, 15809-15812.

(48) Scott, D. J.; Fuchter, M. J.; Ashley, A. E. Nonmetal Catalyzed Hydrogenation of Carbonyl Compounds. J. Am. Chem. Soc. 2014, 136, 15813-15816.

(49) Gyömöre, Á.; Bakos, M.; Földes, T.; Pápai, I.; Domján, A.; Soós, T. Moisture-Tolerant Frustrated Lewis Pair Catalyst for Hydrogenation of Aldehydes and Ketones. ACS Catal. 2015, 5, 5366-5372.

(50) Mahdi, T.; Heiden, Z. M.; Grimme, S.; Stephan, D. W. Metal-Free Aromatic Hydrogenation: Aniline to Cyclohexyl-amine Derivatives. J. Am. Chem. Soc. 2012, 134, 4088-4091.

(51) Longobardi, L. E.; Mahdi, T.; Stephan, D. W. B $\left(\mathrm{C}_{6} \mathrm{~F}_{5}\right)_{3}$ mediated arene hydrogenation/transannulation of para-methoxyanilines. Dalton. Trans. 2015, 44, 7114-7117. 
(52) Li, G.; Liu, Y.; Du, H. B( $\left(\mathrm{C}_{6} \mathrm{~F}_{5}\right)_{3}$-catalyzed metal-free hydrogenation of naphthylamines. Org. Biomol. Chem. 2015, 13, 2875-2878.

(53) Wang, W.; Meng, W.; Du, H. B( $\left.\mathrm{C}_{6} \mathrm{~F}_{5}\right)_{3}$-catalyzed metal-free hydrogenation of 3,6-diarylpyridazines. Dalton. Trans. 2016, 45, 5945-5948.

(54) Mahdi, T.; del Castillo, J. N.; Stephan, D. W. Metal-Free Hydrogenation of N-Based Heterocycles. Organometallics 2013, 32, 1971-1978.

(55) Liu, Y.; Du, H. Metal-Free Borane-Catalyzed Highly Stereoselective Hydrogenation of Pyridines. J. Am. Chem. Soc. 2013, 135, 12968-12971.

(56) Lam, J.; Szkop, K. M.; Mosaferi, E.; Stephan, D. W. FLP catalysis: main group hydrogenations of organic unsaturated substrates. Chem. Soc. Rev. 2018.

(57) Rokob, T. A.; Hamza, A.; Stirling, A.; Pápai, I. On the Mechanism of $B\left(\mathrm{C}_{6} \mathrm{~F}_{5}\right)_{3}$-Catalyzed Direct Hydrogenation of Imines: Inherent and Thermally Induced Frustration. J. Am. Chem. Soc. 2009, 131, 2029-2036.

(58) Farrell, J. M.; Hatnean, J. A.; Stephan, D. W. Activation of Hydrogen and Hydrogenation Catalysis by a Borenium Cation. J. Am. Chem. Soc. 2012, 134, 15728-15731.

(59) Scott, D. J.; Fuchter, M. J.; Ashley, A. E. Designing effective 'frustrated Lewis pair' hydrogenation catalysts. Chem. Soc. Rev. 2017, 46, 5689-5700.

(60) Clark, E. R.; Del Grosso, A.; Ingleson, M. J. The Hydride-Ion Affinity of Borenium Cations and Their Propensity to Activate $\mathrm{H}_{2}$ in Frustrated Lewis Pairs. Chem. Eur. J. 2013, 19, 2462-2466.

(61) Farrell, J. M.; Posaratnanathan, R. T.; Stephan, D. W. A family of N-heterocyclic carbene-stabilized borenium ions for metal-free imine hydrogenation catalysis. Chem. Sci. 2015, 6, 2010-2015.

(62) Noyori, R.; Koizumi, M.; Ishii, D.; Ohkuma, T.: Asymmetric hydrogenation via architectural and functional molecular engineering. In Pure and Applied Chemistry, 2001; Vol. 73; pp 227.

(63) Noyori, R.; Ohkuma, T. Asymmetric Catalysis by Architectural and Functional Molecular Engineering: Practical Chemo- and Stereoselective Hydrogenation of Ketones. Angew. Chem. Int. Ed. 2001, 40, 4073.

(64) Ghattas, G.; Chen, D.; Pan, F.; Klankermayer, J. Asymmetric hydrogenation of imines with a recyclable chiral frustrated Lewis pair catalyst. Dalton. Trans. 2012, 41, 9026-9028.

(65) Lam, J.; Günther, B. A. R.; Farrell, J. M.; Eisenberger, P.; Bestvater, B. P.; Newman, P. D.; Melen, R. L.; Crudden, C. M.; Stephan, D. W. Chiral carbene-borane adducts: precursors for borenium catalysts for asymmetric FLP hydrogenations. Dalton. Trans. 2016, 45, 15303-15316.

(66) Chen, D.; Klankermayer, J. Metal-free catalytic hydrogenation of imines with tris(perfluorophenyl)borane. Chem. Commun. 2008, 2130-2131.

(67) Chen, D.; Klankermayer, J.: Frustrated Lewis Pairs: From Dihydrogen Activation to Asymmetric Catalysis. In Frustrated Lewis Pairs II: Expanding the Scope; Erker, G., Stephan, D. W., Eds.; Springer Berlin Heidelberg: Berlin, Heidelberg, 2013; pp 1-26.

(68) Liu, Y.; Du, H. Chiral Dienes as "Ligands" for Borane-Catalyzed Metal-Free Asymmetric Hydrogenation of Imines. J. Am. Chem. Soc. 2013, 135, 6810-6813.

(69) Lindqvist, M.; Borre, K.; Axenov, K.; Kótai, B.; Nieger, M.; Leskelä, M.; Pápai, I.; Repo, T. Chiral Molecular Tweezers: Synthesis and Reactivity in Asymmetric Hydrogenation. J. Am. Chem. Soc. 2015, 137, 40384041.

(70) Sumerin, V.; Chernichenko, K.; Nieger, M.; Leskelä, M.; Rieger, B.; Repo, T. Highly Active MetalFree Catalysts for Hydrogenation of Unsaturated Nitrogen-Containing Compounds. Adv. Synth. Catal. 2011, 353, 2093-2110.

(71) Wei, S.; Du, H. A Highly Enantioselective Hydrogenation of Silyl Enol Ethers Catalyzed by Chiral Frustrated Lewis Pairs. J. Am. Chem. Soc. 2014, 136, 12261-12264.

(72) Farrell, J. M.; Heiden, Z. M.; Stephan, D. W. Metal-Free Transfer Hydrogenation Catalysis by B(C6F5)3. Organometallics 2011, 30, 4497-4500.

(73) Greb, L.; Oña-Burgos, P.; Schirmer, B.; Grimme, S.; Stephan, D. W.; Paradies, J. Metal-free Catalytic Olefin Hydrogenation: Low-Temperature H2 Activation by Frustrated Lewis Pairs. Angew. Chem. Int. Ed. 2012, 51, 10164-10168.

(74) Greb, L.; Tussing, S.; Schirmer, B.; Oña-Burgos, P.; Kaupmees, K.; Lõkov, M.; Leito, I.; Grimme, S.; Paradies, J. Electronic effects of triarylphosphines in metal-free hydrogen activation: a kinetic and computational study. Chem. Sci. 2013, 4, 2788-2796.

(75) Segawa, Y.; Stephan, D. W. Metal-free hydrogenation catalysis of polycyclic aromatic hydrocarbons. Chem. Commun. 2012, 48, 11963-11965.

(76) Hounjet, L. J.; Bannwarth, C.; Garon, C. N.; Caputo, C. B.; Grimme, S.; Stephan, D. W. Combinations of Ethers and $\mathrm{B}\left(\mathrm{C}_{6} \mathrm{~F}_{5}\right)_{3}$ Function as Hydrogenation Catalysts. Angew. Chem. Int. Ed. 2013, 52, 74927495.

(77) Scott, D. J.; Fuchter, M. J.; Ashley, A. E. Metal-Free Hydrogenation Catalyzed by an Air-Stable Borane: Use of Solvent as a Frustrated Lewis Base. Angew. Chem. Int. Ed. 2014, 53, 10218-10222.

(78) Paradies, J. Metal-Free Hydrogenation of Unsaturated Hydrocarbons Employing Molecular Hydrogen. Angew. Chem. Int. Ed. 2014, 53, 3552-3557. 
27.

(79) Eisenstein, O.; Crabtree, R. H. Outer sphere hydrogenation catalysis. New J. Chem. 2013, 37, 21-

(80) Chernichenko, K.; Madarász, Á.; Pápai, I.; Nieger, M.; Leskelä, M.; Repo, T. A frustrated-Lewis-pair approach to catalytic reduction of alkynes to cis-alkenes. Nat. Chem. 2013, 5, 718.

(81) Oro, L. A.; Carmona, D.: Rhodium. In The Handbook of Homogeneous Hydrogenation; J. G. de Vries, C. J. E., Ed., 2008. Ed., 2008.

(82) Crabtree, R. H.: Iridium. In The Handbook of Homogeneous Hydrogenation; J. G. de Vries, C. J. E.,

(83) Szeto, K. C.; Sahyoun, W.; Merle, N.; Castelbou, J. L.; Popoff, N.; Lefebvre, F.; Raynaud, J.; Godard, C.; Claver, C.; Delevoye, L.; Gauvin, R. M.; Taoufik, M. Development of silica-supported frustrated Lewis pairs: highly active transition metal-free catalysts for the Z-selective reduction of alkynes. Catal. Sci. Technol. 2016, 6, 882-889.

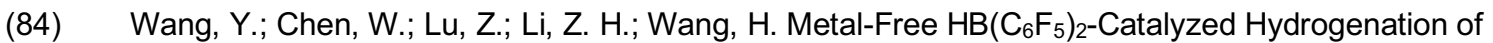
Unfunctionalized Olefins and Mechanism Study of Borane-Mediated $\sigma$-Bond Metathesis. Angew. Chem. Int. Ed. 2013, 52, 7496-7499.

(85) Harhausen, M.; Fröhlich, R.; Kehr, G.; Erker, G. Reactions of Modified Intermolecular Frustrated P/B Lewis Pairs with Dihydrogen, Ethene, and Carbon Dioxide. Organometallics 2012, 31, 2801-2809.

(86) Stirling, A.; Hamza, A.; Rokob, T. A.; Pápai, I. Concerted attack of frustrated Lewis acid-base pairs on olefinic double bonds: a theoretical study. Chem. Commun. 2008, 3148-3150.

(87) Mömming, C. M.; Frömel, S.; Kehr, G.; Fröhlich, R.; Grimme, S.; Erker, G. Reactions of an Intramolecular Frustrated Lewis Pair with Unsaturated Substrates: Evidence for a Concerted Olefin Addition Reaction. J. Am. Chem. Soc. 2009, 131, 12280-12289.

$675-704$

(88) Müller, T. E.; Beller, M. Metal-Initiated Amination of Alkenes and Alkynes. Chem. Rev. 1998, 98,

(89) Zhao, X.; Stephan, D. W. Olefin-Borane "van der Waals Complexes": Intermediates in Frustrated Lewis Pair Addition Reactions. J. Am. Chem. Soc. 2011, 133, 12448-12450.

(90) Zhao, X.; Stephan, D. W. Frustrated Lewis pair olefin addition reactions: P-, N-, C- and H-based nucleophilic additions to an olefin-tethered borane. Chem. Sci. 2012, 3, 2123-2132.

(91) Dureen, M. A.; Brown, C. C.; Stephan, D. W. Deprotonation and Addition Reactions of Frustrated Lewis Pairs with Alkynes. Organometallics 2010, 29, 6594-6607.

(92) Chen, C.; Eweiner, F.; Wibbeling, B.; Fröhlich, R.; Senda, S.; Ohki, Y.; Tatsumi, K.; Grimme, S.; Kehr, G.; Erker, G. Exploring the Limits of Frustrated Lewis Pair Chemistry with Alkynes: Detection of a System that Favors 1,1-Carboboration over Cooperative 1,2-P/B-Addition. Chem. Asain. J. 2010, 5, 2199-2208.

(93) Jiang, C.; Blacque, O.; Berke, H. Activation of Terminal Alkynes by Frustrated Lewis Pairs. Organometallics 2010, 29, 125-133.

(94) Walkinshaw, A. J.; Xu, W.; Suero, M. G.; Gaunt, M. J. Copper-Catalyzed Carboarylation of Alkynes via Vinyl Cations. J. Am. Chem. Soc. 2013, 135, 12532-12535.

(95) Kehr, G.; Erker, G. 1,1-Carboboration. Chem. Commun. 2012, 48, 1839-1850.

(96) Chen, C.; Kehr, G.; Fröhlich, R.; Erker, G. Carbon-Carbon Bond Activation by 1,1-Carboboration of Internal Alkynes. J. Am. Chem. Soc. 2010, 132, 13594-13595.

(97) Feldmann, A.; Kehr, G.; Daniliuc, C. G.; Mück-Lichtenfeld, C.; Erker , G. Functionalization of Intramolecular Frustrated Lewis Pairs by 1,1-Carboboration with Conjugated Enynes. Chem. Eur. J. 2015, 21, 1245612464.

(98) Dureen, M. A.; Stephan, D. W. Terminal Alkyne Activation by Frustrated and Classical Lewis Acid/Phosphine Pairs. J. Am. Chem. Soc. 2009, 131, 8396-8397.

(99) Trost, B. M.; McClory, A. Metal Vinylidenes as Catalytic Species in Organic Reactions. Chem.

Asain. J. 2008, 3, 164-194.

(100) Dureen, M. A.; Brown, C. C.; Stephan, D. W. Addition of Enamines or Pyrroles and B(C6F5)3 "Frustrated Lewis Pairs" to Alkynes. Organometallics 2010, 29, 6422-6432.

(101) Mahdi, T.; Stephan, D. W. Frustrated Lewis Pair Catalyzed Hydroamination of Terminal Alkynes. Angew. Chem. Int. Ed. 2013, 52, 12418-12421.

(102) Mahdi, T.; Stephan, D. W. Stoichiometric and Catalytic Inter- and Intramolecular Hydroamination of Terminal Alkynes by Frustrated Lewis Pairs. Chem. Eur. J. 2015, 21, 11134-11142.

(103) Fürstner, A.; Davies, P. W. Catalytic Carbophilic Activation: Catalysis by Platinum and Gold $\pi$ Acids. Angew. Chem. Int. Ed. 2007, 46, 3410-3449.

(104) McGough, J. S.; Butler, S. M.; Cade, I. A.; Ingleson, M. J. Highly selective catalytic transhydroboration of alkynes mediated by borenium cations and $\mathrm{B}\left(\mathrm{C}_{6} \mathrm{~F}_{5}\right)_{3}$. Chem. Sci. 2016, 7, 3384-3389.

(105) Fleige, M.; Möbus, J.; vom Stein, T.; Glorius, F.; Stephan, D. W. Lewis acid catalysis: catalytic hydroboration of alkynes initiated by Piers' borane. Chem. Commun. 2016, 52, 10830-10833.

(106) Fan, L.; Stephan, D. W. PNPCB heterocycles via thermal and Lewis acid catalyzed transhydroborations. Dalton. Trans. 2016, 45, 9229-9234. 
(107) Wang, T.; Jentgens, X.; Daniliuc, C. G.; Kehr, G.; Erker, G. Tris(pentafluorophenyl)boraneCatalyzed Reaction of Phosphorus/Boron and Nitrogen/Boron Frustrated Lewis Pair Dihydrogen Activation Products with Alkenes and Alkynes. ChemCatChem 2017, 9, 651-658.

(108) Vasko, P.; Zulkifly, I. A.; Fuentes, M. Á.; Mo, Z.; Hicks, J.; Kamer, P. C. J.; Aldridge, S. Reversible C-H Activation, Facile C-B/B-H Metathesis and Apparent Hydroboration Catalysis by a Dimethylxanthene-Based Frustrated Lewis Pair. Chem. Eur. J. 2018, 24, 10531-10540.

(109) Shimoi, M.; Watanabe, T.; Maeda, K.; Curran, D. P.; Taniguchi, T. Radical trans-Hydroboration of Alkynes with N-Heterocyclic Carbene Boranes. Angew. Chem. 2018, 130, 9629-9634.

(110) Lawson, J. R.; Wilkins, L. C.; Melen, R. L. Tris(2,4,6-trifluorophenyl)borane: An Efficient Hydroboration Catalyst. Chem. Eur. J. 2017, 23, 10997-11000.

(111) Hansmann, M. M.; Melen, R. L.; Rominger, F.; Hashmi, A. S. K.; Stephan, D. W. Activation of Alkynes with $\mathrm{B}\left(\mathrm{C}_{6} \mathrm{~F}_{5}\right)_{3}$ - Boron Allylation Reagents Derived from Propargyl Esters. J. Am. Chem. Soc. 2014, 136, 777-782

(112) Hansmann, M. M.; Melen, R. L.; Rominger, F.; Hashmi, A. S. K.; Stephan, D. W. B $\left(\mathrm{C}_{6} \mathrm{~F}_{5}\right)_{3}$ promoted cyclisation of internal propargyl esters: structural characterisation of 1,3-dioxolium compounds. Chem. Commun. 2014, 50, 7243-7245.

(113) Melen, R. L.; Hansmann, M. M.; Lough, A. J.; Hashmi, A. S. K.; Stephan, D. W. Cyclisation versus 1,1-Carboboration: Reactions of $\mathrm{B}\left(\mathrm{C}_{6} \mathrm{~F}_{5}\right)_{3}$ with Propargyl Amides. Chem. Eur. J. 2013, 19, 11928-11938.

(114) Lawson, J. R.; Melen, R. L. Tris(pentafluorophenyl)borane and Beyond: Modern Advances in Borylation Chemistry. Inorg. Chem. 2017, 56, 8627-8643.

(115) Tamke, S.; Qu, Z.-W.; Sitte, N. A.; Flörke, U.; Grimme, S.; Paradies, J. Frustrated Lewis PairCatalyzed Cycloisomerization of 1,5-Enynes via a 5-endo-dig Cyclization/Protodeborylation Sequence. Angew. Chem. Int. Ed. 2016, 55, 4336-4339.

(116) Yuan, K.; Wang, S. trans-Aminoboration across Internal Alkynes Catalyzed by $B\left(\mathrm{C}_{6} \mathrm{~F}_{5}\right)_{3}$ for the Synthesis of Borylated Indoles. Org. Lett. 2017, 19, 1462-1465.

(117) Hansmann, M. M.; Melen, R. L.; Rudolph, M.; Rominger, F.; Wadepohl, H.; Stephan, D. W.;

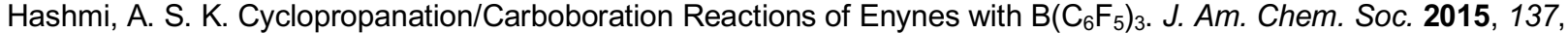
15469-15477.

(118) Wilkins, L. C.; Wieneke, P.; Newman, P. D.; Kariuki, B. M.; Rominger, F.; Hashmi, A. S. K.; Hansmann, M. M.; Melen, R. L. Pathways to Functionalized Heterocycles: Propargyl Rearrangement using $B\left(C_{6} F_{5}\right)_{3}$. Organometallics 2015, 34, 5298-5309.

(119) Wilkins, L. C.; Hamilton, H. B.; Kariuki, B. M.; Hashmi, A. S. K.; Hansmann, M. M.; Melen, R. L. Lewis acid-base 1,2-addition reactions: synthesis of pyrylium borates from en-ynoate precursors. Dalton. Trans. 2016, 45, 5929-5932.

(120) Wilkins, L. C.; Lawson, J. R.; Wieneke, P.; Rominger, F.; Hashmi, A. S. K.; Hansmann, M. M.; Melen, R. L. The Propargyl Rearrangement to Functionalised Allyl-Boron and Borocation Compounds. Chem. Eur. J. 2016, 22, 14618-14624.

(121) Wilkins, L. C.; Günther, B. A. R.; Walther, M.; Lawson, J. R.; Wirth, T.; Melen, R. L. Contrasting Frustrated Lewis Pair Reactivity with Selenium- and Boron-Based Lewis Acids. Angew. Chem. Int. Ed. 2016, 55, $11292-11295$.

(122) Issaian, A.; Tu, K. N.; Blum, S. A. Boron-Heteroatom Addition Reactions via Borylative Heterocyclization: Oxyboration, Aminoboration, and Thioboration. Acc. Chem. Res. 2017, 50, 2598-2609.

(123) Wang, T.; Daniliuc, C. G.; Mück-Lichtenfeld, C.; Kehr, G.; Erker, G. Formation of Reactive mConjugated Frustrated N/B Pairs by Borane-Induced Propargyl Amine Rearrangement. J. Am. Chem. Soc. 2018, 140, 3635-3643.

(124) Lawson, J. R.; Melen, R. L.: Recent developments and applications of Lewis acidic boron reagents. In Organometallic Chemistry: Volume 41; The Royal Society of Chemistry, 2017; Vol. 41; pp 1-27.

(125) Soltani, Y.; Wilkins, L. C.; Melen, R. L. Stoichiometric and Catalytic C-C and C-H Bond Formation with B(C6F5)3 via Cationic Intermediates. Angew. Chem. Int. Ed. 2017, 56, 11995-11999.

(126) Burg, A. B.; Schlesinger, H. I. Hydrides of Boron. VII. Evidence of the Transitory Existence of Borine $\left(\mathrm{BH}_{3}\right)$ : Borine Carbonyl and Borine Trimethylammine. J. Am. Chem. Soc. 1937, 59, 780-787.

(127) Sajid, M.; Elmer, L.-M.; Rosorius, C.; Daniliuc, C. G.; Grimme, S.; Kehr, G.; Erker, G. Facile Carbon Monoxide Reduction at Intramolecular Frustrated Phosphane/Borane Lewis Pair Templates. Angew. Chem. Int. Ed. 2013, 52, 2243-2246.

(128) Sajid, M.; Kehr, G.; Daniliuc, C. G.; Erker, G. Formylborane Formation with Frustrated Lewis Pair Templates. Angew. Chem. Int. Ed. 2014, 53, 1118-1121.

(129) Finze, M.; Bernhardt, E.; Terheiden, A.; Berkei, M.; Willner, H.; Christen, D.; Oberhammer, H.; Aubke, F. Tris(trifluoromethyl)borane Carbonyl, $\left(\mathrm{CF}_{3}\right)_{3} \mathrm{BCOSynthesis,} \mathrm{Physical,} \mathrm{Chemical} \mathrm{and} \mathrm{Spectroscopic}$ Properties, Gas Phase, and Solid State Structure. J. Am. Chem. Soc. 2002, 124, 15385-15398.

(130) Finze, M.; Bernhardt, E.; Willner, H.; Lehmann, C. W. Reactions of $\left(\mathrm{CF}_{3}\right)_{3} \mathrm{BCO}$ with Amines and Phosphines. Inorg. Chem. 2006, 45, 669-678. 
(131) Gerken, M.; Pawelke, G.; Bernhardt, E.; Willner, H. Syntheses and Characterization of $\left(\mathrm{C}_{2} \mathrm{~F}_{5}\right)_{3} \mathrm{BCO}$ and $\left(\mathrm{C}_{3} \mathrm{~F}_{7}\right)_{3} \mathrm{BCO}$. Chem. Eur. J. 2010, 16, 7527-7536.

(132) Fukazawa, A.; Dutton, J. L.; Fan, C.; Mercier, L. G.; Houghton, A. Y.; Wu, Q.; Piers, W. E.; Parvez, M. Reaction of pentaarylboroles with carbon monoxide: an isolable organoboron carbonyl complex. Chem. Sci. 2012, 3, 1814-1818.

(133) Goldman, A. S.; Krogh-Jespersen, K. Why Do Cationic Carbon Monoxide Complexes Have High C-O Stretching Force Constants and Short C-O Bonds? Electrostatic Effects, Not $\sigma$-Bonding. J. Am. Chem. Soc. 1996, 118, $12159-12166$.

(134) Sajid, M.; Lawzer, A.; Dong, W.; Rosorius, C.; Sander, W.; Schirmer, B.; Grimme, S.; Daniliuc, C. G.; Kehr, G.; Erker, G. Carbonylation Reactions of Intramolecular Vicinal Frustrated Phosphane/Borane Lewis Pairs. J. Am. Chem. Soc. 2013, 135, 18567-18574.

(135) Elmer, L.-M.; Kehr, G.; Daniliuc, C. G.; Siedow, M.; Eckert, H.; Tesch, M.; Studer, A.; Williams, K.; Warren, T. H.; Erker, G. The Chemistry of a Non-Interacting Vicinal Frustrated Phosphane/Borane Lewis Pair. Chem. Eur. J. 2017, 23, 6056-6068.

(136) Cabrera, A. R.; Rojas, R. S.; Valderrama, M.; Plüss, P.; Berke, H.; Daniliuc, C. G.; Kehr, G.; Erker, G. Synthesis of new asymmetric substituted boron amidines - reactions with $\mathrm{CO}$ and transfer hydrogenations of phenylacetylene. Dalton. Trans. 2015, 44, 19606-19614.

(137) Wang, L.; Dong, S.; Daniliuc, C. G.; Liu, L.; Grimme, S.; Knitsch, R.; Eckert, H.; Hansen, M. R.; Kehr, G.; Erker, G. Formation of macrocyclic ring systems by carbonylation of trifunctional P/B/B frustrated Lewis pairs. Chem. Sci. 2018, 9, 1544-1550.

(138) Brown, H. C. Organoborane-carbon monoxide reactions. Synthesis of carbon structures. Acc. Chem. Res. 1969, 2, 65-72.

(139) Franke, R.; Selent, D.; Börner, A. Applied Hydroformylation. Chem. Rev. 2012, 112, 5675-5732.

(140) Masters, C.: The Fischer-Tropsch Reaction. In Advances in Organometallic Chemistry; Stone, F. G. A., West, R., Eds.; Academic Press, 1979; Vol. 17; pp 61-103.

(141) Maitlis, P. M.; Zanotti, V. The role of electrophilic species in the Fischer-Tropsch reaction. Chem. Commun. 2009, 1619-1634.

(142) Dobrovetsky, R.; Stephan, D. W. Stoichiometric Metal-Free Reduction of CO in Syn-Gas. J. Am. Chem. Soc. 2013, 135, 4974-4977.

(143) Yu, J.; Kehr, G.; Daniliuc, C. G.; Erker, G. Cooperative reaction chemistry derived from a boratadiene framework. Chem. Commun. 2016, 52, 1393-1396.

(144) Jian, Z.; Kehr, G.; Daniliuc, C. G.; Wibbeling, B.; Wiegand, T.; Siedow, M.; Eckert, H.; Bursch, M.; Grimme, S.; Erker, G. CO-Reduction Chemistry: Reaction of a CO-Derived Formylhydridoborate with Carbon Monoxide, with Carbon Dioxide, and with Dihydrogen. J. Am. Chem. Soc. 2017, 139, 6474-6483.

(145) Wang, L.; Zhang, S.; Hasegawa, Y.; Daniliuc, C. G.; Kehr, G.; Erker, G. Cooperative carbon monoxide to formyl reduction at a trifunctional PBB frustrated Lewis pair. Chem. Commun. 2017, 53, 5499-5502.

(146) Ye, K.-Y.; Daniliuc, C. G.; Dong, S.; Kehr, G.; Erker, G. Frustrated Lewis Pair Behavior of an Open, Noninteracting Phosphane/Borane Pair at a Rigid Organic Framework: Exploring Decisive Factors for FLP Activity. Organometallics 2017, 36, 5003-5012.

(147) Wang, T.; Kehr, G.; Daniliuc, C. G.; Erker, G. Metal-Free Carbonylation Route to a Reactive Borataepoxide System. Organometallics 2018, 37, 1040-1049.

(148) Wang, T.; Wang, L.; Daniliuc, C. G.; Samigullin, K.; Wagner, M.; Kehr, G.; Erker, G. CO/CO and NO/NO coupling at a hidden frustrated Lewis pair template. Chem. Sci. 2017, 8, 2457-2463.

(149) Del Grosso, A.; Singleton, P. J.; Muryn, C. A.; Ingleson, M. J. Pinacol Boronates by Direct Arene Borylation with Borenium Cations. Angew. Chem. Int. Ed. 2011, 50, 2102-2106.

(150) Farrell, J. M.; Stephan, D. W. Planar N-Heterocyclic Carbene Diarylborenium lons: Synthesis by Cationic Borylation and Reactivity with Lewis Bases. Angew. Chem. Int. Ed. 2015, 54, 5214-5217.

(151) Prokofjevs, A.; Kampf, J. W.; Vedejs, E. A Boronium Ion with Exceptional Electrophilicity. Angew. Chem. Int. Ed. 2011, 50, 2098-2101.

(152) Del Grosso, A.; Pritchard, R. G.; Muryn, C. A.; Ingleson, M. J. Chelate Restrained Boron Cations for Intermolecular Electrophilic Arene Borylation. Organometallics 2010, 29, 241-249.

(153) De Vries, T. S.; Prokofjevs, A.; Harvey, J. N.; Vedejs, E. Superelectrophilic Intermediates in Nitrogen-Directed Aromatic Borylation. J. Am. Chem. Soc. 2009, 131, 14679-14687.

(154) Prokofjevs, A.; Vedejs, E. N-Directed Aliphatic C-H Borylation Using Borenium Cation Equivalents. J. Am. Chem. Soc. 2011, 133, 20056-20059.

(155) Grosso, A. D.; Helm, M. D.; Solomon, S. A.; Caras-Quintero, D.; Ingleson, M. J. Simple inexpensive boron electrophiles for direct arene borylation. Chem. Commun. 2011, 47, 12459-12461.

(156) Solomon, S. A.; Del Grosso, A.; Clark, E. R.; Bagutski, V.; McDouall, J. J. W.; Ingleson, M. J. Reactivity of Lewis Acid Activated Diaza- and Dithiaboroles in Electrophilic Arene Borylation. Organometallics 2012 31, 1908-1916. 
(157) Bagutski, V.; Del Grosso, A.; Carrillo, J. A.; Cade, I. A.; Helm, M. D.; Lawson, J. R.; Singleton, P. J.; Solomon, S. A.; Marcelli, T.; Ingleson, M. J. Mechanistic Studies into Amine-Mediated Electrophilic Arene Borylation and Its Application in MIDA Boronate Synthesis. J. Am. Chem. Soc. 2013, 135, 474-487.

(158) Prokofjevs, A.; Jermaks, J.; Borovika, A.; Kampf, J. W.; Vedejs, E. Electrophilic C-H Borylation and Related Reactions of B-H Boron Cations. Organometallics 2013, 32, 6701-6711.

(159) McGough, J. S.; Cid, J.; Ingleson, M. J. Catalytic Electrophilic C-H Borylation Using NHC·Boranes and lodine Forms C2-, not C3-, Borylated Indoles. Chem. Eur. J. 2017, 23, 8180-8184.

(160) Farrell, J. M.; Schmidt, D.; Grande, V.; Würthner, F. Synthesis of a Doubly Boron-Doped Perylene through NHC-Borenium Hydroboration/C-H Borylation/Dehydrogenation. Angew. Chem. Int. Ed. 2017, 56, 1184611850 .

(161) Yin, Q.; Klare, H. F. T.; Oestreich, M. Catalytic Friedel-Crafts C-H Borylation of Electron-Rich Arenes: Dramatic Rate Acceleration by Added Alkenes. Angew. Chem. Int. Ed. 2017, 56, 3712-3717.

(162) Zhang, S.; Han, Y.; He, J.; Zhang, Y. B $\left(\mathrm{C}_{6} \mathrm{~F}_{5}\right)_{3}$-Catalyzed C3-Selective C-H Borylation of Indoles: Synthesis, Intermediates, and Reaction Mechanism. J. Org. Chem. 2018, 83, 1377-1386.

(163) Liu, Y.-L.; Kehr, G.; Daniliuc, C. G.; Erker, G. Metal-Free Arene and Heteroarene Borylation Catalyzed by Strongly Electrophilic Bis-boranes. Chem. Eur. J. 2017, 23, 12141-12144.

(164) Larsen, M. A.; Hartwig, J. F. Iridium-Catalyzed C-H Borylation of Heteroarenes: Scope, Regioselectivity, Application to Late-Stage Functionalization, and Mechanism. J. Am. Chem. Soc. 2014, 136, 42874299.

(165) Ackermann, L. Carboxylate-Assisted Transition-Metal-Catalyzed C-H Bond Functionalizations: Mechanism and Scope. Chem. Rev. 2011, 111, 1315-1345.

(166) Légaré, M.-A.; Courtemanche, M.-A.; Rochette, É.; Fontaine, F.-G. Metal-free catalytic C-H bond activation and borylation of heteroarenes. Science 2015, 349, 513-516.

(167) Légaré Lavergne, J.; Jayaraman, A.; Misal Castro, L. C.; Rochette, É.; Fontaine, F.-G. Metal-Free Borylation of Heteroarenes Using Ambiphilic Aminoboranes: On the Importance of Sterics in Frustrated Lewis Pair CH Bond Activation. J. Am. Chem. Soc. 2017, 139, 14714-14723.

(168) Shao, Y.; Zhang, J.; Li, Y.; Liu, Y.; Ke, Z. Frustrated Lewis Pair Catalyzed C-H Activation of Heteroarenes: A Stepwise Carbene Mechanism Due to Distance Effect. Org. Lett. 2018, 20, 1102-1105.

(169) Légaré, M.-A.; Rochette, É.; Légaré Lavergne, J.; Bouchard, N.; Fontaine, F.-G. Bench-stable frustrated Lewis pair chemistry: fluoroborate salts as precatalysts for the $\mathrm{C}-\mathrm{H}$ borylation of heteroarenes. Chem. Commun. 2016, 52, 5387-5390.

(170) Chernichenko, K.; Lindqvist, M.; Kótai, B.; Nieger, M.; Sorochkina, K.; Pápai, I.; Repo, T. MetalFree sp2-C-H Borylation as a Common Reactivity Pattern of Frustrated 2-Aminophenylboranes. J. Am. Chem. Soc. 2016, 138, 4860-4868.

(171) Wu, D.; Kong, L.; Li, Y.; Ganguly, R.; Kinjo, R. 1,3,2,5-Diazadiborinine featuring nucleophilic and electrophilic boron centres. Nat. Commun. 2015, 6, 7340.

(172) Wu, D.; Ganguly, R.; Li, Y.; Hoo, S. N.; Hirao, H.; Kinjo, R. Reversible [4 + 2] cycloaddition reaction of 1,3,2,5-diazadiborinine with ethylene. Chem. Sci. 2015, 6, 7150-7155.

(173) Wang, B.; Li, Y.; Ganguly, R.; Hirao, H.; Kinjo, R. Ambiphilic boron in 1,4,2,5-diazadiborinine. Nat. Commun. 2016, 7, 11871.

(174) Su, Y.; Li, Y.; Ganguly, R.; Kinjo, R. Engineering the Frontier Orbitals of a Diazadiborinine for Facile Activation of $\mathrm{H}_{2}, \mathrm{NH}_{3}$, and an Isonitrile. Angew. Chem. Int. Ed. 2018, 57, 7846-7849.

(175) Taylor, J. W.; McSkimming, A.; Guzman, C. F.; Harman, W. H. N-Heterocyclic Carbene-Stabilized Boranthrene as a Metal-Free Platform for the Activation of Small Molecules. J. Am. Chem. Soc. 2017, 139, 1103211035.

(176) von Grotthuss, E.; Diefenbach, M.; Bolte, M.; Lerner, H.-W.; Holthausen, M. C.; Wagner, M. Reversible Dihydrogen Activation by Reduced Aryl Boranes as Main-Group Ambiphiles. Angew. Chem. Int. Ed. 2016, $55,14067-14071$.

(177) Lorbach, A.; Bolte, M.; Lerner, H.-W.; Wagner, M. Dilithio 9,10-Diborataanthracene: Molecular Structure and 1,4-Addition Reactions. Organometallics 2010, 29, 5762-5765.

(178) Urry, G.; Wartik, T.; Moore, R. E.; Schlesinger, H. I. The Preparation and Some of the Properties of Diboron Tetrachloride, $\mathrm{B}_{2} \mathrm{Cl}_{4}$. J. Am. Chem. Soc. 1954, 76, 5293-5298.

(179) Ahmed, L.; Castillo, J.; Saulys, D. A.; Morrison, J. A. Reactivity of the diboron tetrahalides. Diboration of ethylene with diboron tetrabromide and thermal decomposition and ligand exchanges of diboron tetrabromide and diboron tetrachloride in carbon tetrabromide and carbon tetrachloride. Inorg. Chem. 1992, 31, 706710.

(180) Ceron, P.; Finch, A.; Frey, J.; Kerrigan, J.; Parsons, T.; Urry, G.; Schlesinger, H. I. Diboron Tetrachloride and Tetrafluoride as Reagents for the Synthesis of Organoboron Compounds. II. The Behavior of the Diboron Tetrahalides toward Unsaturated Organic Compounds. J. Am. Chem. Soc. 1959, 81, 6368-6371.

(181) Urry, G.; Kerrigan, J.; Parsons, T. D.; Schlesinger, H. I. Diboron Tetrachloride, B2Cl4, as a Reagent for the Synthesis of Organo-boron Compounds. I. The Reaction of Diboron Tetrachloride with Ethylene. J. Am. Chem. Soc. 1954, 76, 5299-5301. 
(182) Pubill-Ulldemolins, C.; Fernánez, E.; Bo, C.; Brown, J. M. Origins of observed reactivity and specificity in the addition of $\mathrm{B} 2 \mathrm{Cl} 4$ and analogues to unsaturated compounds. Org. Biomol. Chem. 2015, 13, 96199628.

(183) Neeve, E. C.; Geier, S. J.; Mkhalid, I. A. I.; Westcott, S. A.; Marder, T. B. Diboron(4) Compounds: From Structural Curiosity to Synthetic Workhorse. Chem. Rev. 2016, 116, 9091-9161.

(184) Dewhurst, R. D.; Neeve, E. C.; Braunschweig, H.; Marder, T. B. sp $^{2}-\mathrm{sp}^{3}$ diboranes: astounding structural variability and mild sources of nucleophilic boron for organic synthesis. Chem. Commun. 2015, 51, 95949607.

(185) Tsukahara, N.; Asakawa, H.; Lee, K.-H.; Lin, Z.; Yamashita, M. Cleaving Dihydrogen with Tetra(otolyl)diborane(4). J. Am. Chem. Soc. 2017, 139, 2593-2596.

(186) Yalpani, M.; Köster, R. Partial Hydrogenation: From Anthracene to Coronene. Chem. Ber. 1990, $123,719-724$

(187) Yalpani, M.; Lunow, T.; Köster, R. Reduction of Polycyclic Arenes by -Boranes, II. Borane Catalyzed Hydrogenation of Naphthalenes to Tetralins. Chem. Ber. 1989, 122, 687-693.

(188) Katsuma, Y.; Tsukahara, N.; Wu, L.; Lin, Z.; Yamashita, M. Reaction of $\mathrm{B}_{2}(\mathrm{o} \text {-tol) })_{4}$ with $\mathrm{CO}$ and Isocyanides: Cleavage of the $\mathrm{C} \equiv \mathrm{O}$ Triple Bond and Direct $\mathrm{C}-\mathrm{H}$ Borylations. Angew. Chem. Int. Ed. 2018, 57, 61096114.

(189) Zheng, J.; Li, Z. H.; Wang, H. Addition of dihydrogen to a borylborenium center. Chem. Sci. 2018, $9,1433-1438$

(190) Araki, T.; Hirai, M.; Wakamiya, A.; Piers, W. E.; Yamaguchi, S. Antiaromatic Dithieno-1,2-dihydro1,2-diborin Splits Diatomic Hydrogen. Chem. Lett. 2017, 46, 1714-1717.

(191) Kaese, T.; Trageser, T.; Budy, H.; Bolte, M.; Lerner, H.-W.; Wagner, M. A redox-active diborane platform performs $\mathrm{C}\left(\mathrm{sp}^{3}\right)-\mathrm{H}$ activation and nucleophilic substitution reactions. Chem. Sci. 2018, 9, 3881-3891.

(192) Moezzi, A.; Olmstead, M. M.; Power, P. P. Boron-boron double bonding in the species $\left[\mathrm{B}_{2} \mathrm{R}_{4}\right] 2-$ : synthesis and structure of $\left[\left\{\left(\mathrm{Et}_{2} \mathrm{O}\right) \mathrm{Li}_{2}\left\{\mathrm{Mes}_{2} \mathrm{BB}(\mathrm{Mes}) \mathrm{Ph}\right\}\right]\right.$, a diborane(4) dianion analog of a substituted ethylene. $J$. Am. Chem. Soc. 1992, 114, 2715-2717.

(193) Nöth, H.; Knizek, J.; Ponikwar, W. A Boron-Boron Double Bond in the Dianions of Tetra(amino)diborates. Eur. J. Inorg. Chem. 1999, 1999, 1931-1937.

(194) Wang, Y.; Quillian, B.; Wei, P.; Wannere, C. S.; Xie, Y.; King, R. B.; Schaefer, H. F.; Schleyer, P. v. R.; Robinson, G. H. A Stable Neutral Diborene Containing a BB Double Bond. J. Am. Chem. Soc. 2007, 129, 1241212413.

(195) Wang, Y.; Quillian, B.; Wei, P.; Xie, Y.; Wannere, C. S.; King, R. B.; Schaefer, H. F.; Schleyer, P. v. R.; Robinson, G. H. Planar, Twisted, and Trans-Bent: Conformational Flexibility of Neutral Diborenes. J. Am. Chem. Soc. 2008, 130, 3298-3299.

(196) Braunschweig, H.; Dewhurst, R. D.; Hammond, K.; Mies, J.; Radacki, K.; Vargas, A. AmbientTemperature Isolation of a Compound with a Boron-Boron Triple Bond. Science 2012, 336, 1420-1422.

(197) Braunschweig, H.; Dewhurst, R. D.; Hörl, C.; Phukan, A. K.; Pinzner, F.; Ullrich, S. Direct Hydroboration of B=B Bonds: A Mild Strategy for the Proliferation of B-B Bonds. Angew. Chem. Int. Ed. 2014, 53, 3241-3244.

(198) Braunschweig, H.; Dellermann, T.; Ewing, W. C.; Kramer, T.; Schneider, C.; Ullrich, S. Reductive Insertion of Elemental Chalcogens into Boron-Boron Multiple Bonds. Angew. Chem. Int. Ed. 2015, 54, 10271-10275.

(199) Braunschweig, H.; Constantinidis, P.; Dellermann, T.; Ewing, W. C.; Fischer, I.; Hess, M.; Knight, F. R.; Rempel, A.; Schneider, C.; Ullrich, S.; Vargas, A.; Woollins, J. D. Highly Strained Heterocycles Constructed from Boron-Boron Multiple Bonds and Heavy Chalcogens. Angew. Chem. Int. Ed. 2016, 55, 5606-5609.

(200) Wang, S. R.; Arrowsmith, M.; Böhnke, J.; Braunschweig, H.; Dellermann, T.; Dewhurst, R. D.; Kelch, H.; Krummenacher, I.; Mattock, J. D.; Müssig, J. H.; Thiess, T.; Vargas, A.; Zhang, J. Engineering a Small HOMO-LUMO Gap and Intramolecular C-H Borylation by Diborene/Anthracene Orbital Intercalation. Angew. Chem. Int. Ed. 2017, 56, 8009-8013.

(201) Auerhammer, D.; Arrowsmith, M.; Dewhurst, R. D.; Kupfer, T.; Böhnke, J.; Braunschweig, H. Closely related yet different: a borylene and its dimer are non-interconvertible but connected through reactivity. Chem. Sci. 2018, 9, 2252-2260.

(202) Böhnke, J.; Braunschweig, H.; Dellermann, T.; Ewing, W. C.; Kramer, T.; Krummenacher, I.; Vargas, A. From an Electron-Rich Bis(boraketenimine) to an Electron-Poor Diborene. Angew. Chem. Int. Ed. 2015, $54,4469-4473$.

(203) Böhnke, J.; Brückner, T.; Hermann, A.; González-Belman, O. F.; Arrowsmith, M.; Jiménez-Halla, J. O. C.; Braunschweig, H. Single and double activation of acetone by isolobal $\mathrm{B}=\mathrm{N}$ and $\mathrm{B}=\mathrm{B}$ triple bonds. Chem. Sci. 2018, 9, 5354-5359.

(204) Braunschweig, H.; Dellermann, T.; Dewhurst, R. D.; Ewing, W. C.; Hammond, K.; Jimenez-Halla, J. O. C.; Kramer, T.; Krummenacher, I.; Mies, J.; Phukan, A. K.; Vargas, A. Metal-free binding and coupling of carbon monoxide at a boron-boron triple bond. Nat. Chem. 2013, 5, 1025. 
(205) Böhnke, J.; Braunschweig, H.; Dellermann, T.; Ewing, W. C.; Hammond, K.; Jimenez-Halla, J. O. C.; Kramer, T.; Mies, J. The Synthesis of $\mathrm{B}_{2}(\mathrm{SIDip})_{2}$ and its Reactivity Between the Diboracumulenic and Diborynic Extremes. Angew. Chem. Int. Ed. 2015, 54, 13801-13805.

(206) Böhnke, J.; Braunschweig, H.; Ewing, W. C.; Hörl, C.; Kramer, T.; Krummenacher, I.; Mies, J.; Vargas, A. Diborabutatriene: An Electron-Deficient Cumulene. Angew. Chem. Int. Ed. 2014, 53, 9082-9085.

(207) Arrowsmith, M.; Böhnke, J.; Braunschweig, H.; Celik, M. A.; Dellermann, T.; Hammond, K. Uncatalyzed Hydrogenation of First-Row Main Group Multiple Bonds. Chem. Eur. J. 2016, 22, 17169-17172.

(208) Arrowsmith, M.; Böhnke, J.; Braunschweig, H.; Celik, M. A. Reactivity of a Dihydrodiborene with CO: Coordination, Insertion, Cleavage, and Spontaneous Formation of a Cyclic Alkyne. Angew. Chem. Int. Ed. 2017, $56,14287-14292$.

(209) Böhnke, J.; Arrowsmith, M.; Braunschweig, H. Reactivity Enhancement of a Zerovalent Diboron Compound by Desymmetrization. J. Am. Chem. Soc. 2018, 140, 10368-10373.

(210) Arrowsmith, M.; Braunschweig, H.; Stennett, T. E. Formation and Reactivity of Electron-Precise B-B Single and Multiple Bonds. Angew. Chem. Int. Ed. 2017, 56, 96-115.

(211) March, J.: Advanced Organic Chemistry: Reactions, Mechanisms and Structure; 4th ed.; John Wiley and Sons: New York, 1992. pp. p 861.

(212) McKee, M. L. Ab Initio Study of Rearrangements on the $(\mathrm{CH})_{2}(\mathrm{BR})_{2}, \mathrm{RH}$, and $\mathrm{NH}_{2}$ Potential Energy Surfaces. Inorg. Chem. 2000, 39, 4206-4210.

(213) Hildenbrand, M.; Pritzkow, H.; Siebert, W. 1,2-Bis(diisopropylamino)-1,2-dihydro-1,2-diboret. Angew. Chem. 1985, 97, 769-770.

(214) Boag, N. M.; Green, M.; Howard, J. A. K.; Spencer, J. L.; Stansfield, R. F. D.; Gordon, F.; Stone, A.; Thomas, M. D. O.; Vicente, J.; Woodward, P. Bridging acetylene complexes of nickel and platinum; X-ray crystal structure of $\left[\mathrm{Pt}_{3}\left(\mathrm{Et}_{3} \mathrm{P}\right) 4\left(\mathrm{PhC}_{2} \mathrm{Ph}\right)_{2}\right]$. Journal of the Chemical Society, Chemical Communications 1977, $930-931$.

(215) DeWitt, E. J.; Ramp, F. L.; Trapasso, L. E. HOMOGENEOUS HYDROGENATION CATALYZED BY BORANES. J. Am. Chem. Soc. 1961, 83, 4672-4672.

(216) Ramp, F. L.; DeWitt, E. J.; Trapasso, L. E. Homogeneous Hydrogenation Catalyzed by Boranes. J. Org. Chem. 1962, 27, 4368-4372.

(217) Fan, C.; Mercier, L. G.; Piers, W. E.; Tuononen, H. M.; Parvez, M. Dihydrogen Activation by Antiaromatic Pentaarylboroles. J. Am. Chem. Soc. 2010, 132, 9604-9606.

(218) Houghton, A. Y.; Karttunen, V. A.; Fan, C.; Piers, W. E.; Tuononen, H. M. Mechanistic Studies on the Metal-Free Activation of Dihydrogen by Antiaromatic Pentarylboroles. J. Am. Chem. Soc. 2013, 135, 941-947.

(219) Houghton, A. Y.; Karttunen, V. A.; Piers, W. E.; Tuononen, H. M. Hydrogen activation with perfluorinated organoboranes: 1,2,3-tris(pentafluorophenyl)-4,5,6,7-tetrafluoro-1-boraindene. Chem. Commun. 2014, $50,1295-1298$.

(220) Nikonov, G. I.; Vyboishchikov, S. F.; Shirobokov, O. G. Facile Activation of H-H and Si-H Bonds by Boranes. J. Am. Chem. Soc. 2012, 134, 5488-5491.

(221) Lu, Z.; Cheng, Z.; Chen, Z.; Weng, L.; Li, Z. H.; Wang, H. Heterolytic Cleavage of Dihydrogen by "Frustrated Lewis Pairs" Comprising Bis(2,4,6-tris(trifluoromethyl)phenyl)borane and Amines: Stepwise versus Concerted Mechanism. Angew. Chem. Int. Ed. 2011, 50, 12227-12231.

(222) Waterman, R. б-Bond Metathesis: A 30-Year Retrospective. Organometallics 2013, 32, 7249-7263.

(223) Collman, J. P. H., L. S.; Norton, J. R.; Finke, R. G.: Principles and Applications of Organotransition Metal Chemistry; University Science Books: Mill Valley, CA, 1987.

(224) Yin, Q.; Kemper, S.; Klare, H. F. T.; Oestreich, M. Boron Lewis Acid-Catalyzed Hydroboration of Alkenes with Pinacolborane: BArF3 Does What B(C6F5)3 Cannot Do! Chem. Eur. J. 2016, 22, 13840-13844.

(225) Légaré, M.-A.; Bélanger-Chabot, G.; De Robillard, G.; Languérand, A.; Maron, L.; Fontaine, F.-G. Insights into the Formation of Borabenzene Adducts via Ligand Exchange Reactions and TMSCl Elimination from Boracyclohexadiene Precursors. Organometallics 2014, 33, 3596-3606.

(226) Maier, G.; Reisenauer, H. P.; Henkelmann, J.; Kliche, C. Stickstoff-Fixierung durch Borabenzol. Angew. Chem. 1988, 100, 303-303.

(227) Jin, J.; Wang, G.; Zhou, M.; Andrada, D. M.; Hermann, M.; Frenking, G. The $\left[\mathrm{B}_{3}(\mathrm{NN})_{3}\right]^{+}$and $\left[\mathrm{B}_{3}(\mathrm{CO})_{3}\right]^{+}$Complexes Featuring the Smallest m-Aromatic Species $\mathrm{B}_{3}{ }^{+}$. Angew. Chem. Int. Ed. 2016, 55, $2078-2082$. (228) Zhou, M.; Tsumori, N.; Li, Z.; Fan, K.; Andrews, L.; Xu, Q. OCBBCO: A Neutral Molecule with

Some Boron-Boron Triple Bond Character. J. Am. Chem. Soc. 2002, 124, 12936-12937.

(229) Kinjo, R.; Donnadieu, B.; Celik, M. A.; Frenking, G.; Bertrand, G. Synthesis and Characterization of a Neutral Tricoordinate Organoboron Isoelectronic with Amines. Science 2011, 333, 610-613.

(230) Soleilhavoup, M.; Bertrand, G. Borylenes: An Emerging Class of Compounds. Angew. Chem. Int. Ed. 2017, 56, 10282-10292.

(231) Braunschweig, H.; Dewhurst, R. D.; Gessner, V. H. Transition metal borylene complexes. Chem. Soc. Rev. 2013, 42, 3197-3208.

(232) Liu, S.; Légaré, M.-A.; Auerhammer, D.; Hofmann, A.; Braunschweig, H. The First Boron-Tellurium Double Bond: Direct Insertion of Heavy Chalcogens into a Mn=B Double Bond. Angew. Chem. Int. Ed. 2017, 56, 15760-15763. 
(233) Pranckevicius, C.; Jimenéz-Halla, J. O. C.; Kirsch, M.; Krummenacher, I.; Braunschweig, H. Complexation and Release of N-Heterocyclic Carbene-Aminoborylene Ligands from Group VI and VIII Metals. J. Am. Chem. Soc. 2018, 140, 10524-10529.

(234) Arrowsmith, M.; Auerhammer, D.; Bertermann, R.; Braunschweig, H.; Bringmann, G.; Celik, M. A.; Dewhurst, R. D.; Finze, M.; Grüne, M.; Hailmann, M.; Hertle, T.; Krummenacher, I. Generation of Dicoordinate Boron(I) Units by Fragmentation of a Tetra-Boron(I) Molecular Square. Angew. Chem. Int. Ed. 2016, 55, 1446414468.

(235) Celik, M. A.; Sure, R.; Klein, S.; Kinjo, R.; Bertrand, G.; Frenking, G. Borylene Complexes (BH)L2 and Nitrogen Cation Complexes $\left(\mathrm{N}^{+}\right) \mathrm{L}_{2}$ : Isoelectronic Homologues of Carbones $\mathrm{CL}_{2}$. Chem. Eur. J. 2012, 18, 56765692.

(236) Kong, L.; Ganguly, R.; Li, Y.; Kinjo, R. Diverse reactivity of a tricoordinate organoboron L2PhB: $(L=$ oxazol-2-ylidene) towards alkali metal, group 9 metal, and coinage metal precursors. Chem. Sci. 2015, 6, 2893-2902.

(237) Krasowska, M.; Edelmann, M.; Bettinger, H. F. Electronically Excited States of Borylenes. J. Phys. Chem. A 2016, 120, 6332-6341.

(238) Krasowska, M.; Bettinger, H. F. Reactivity of Borylenes toward Ethyne, Ethene, and Methane. J. Am. Chem. Soc. 2012, 134, 17094-17103.

(239) Edel, K.; Krieg, M.; Grote, D.; Bettinger, H. F. Photoreactions of Phenylborylene with Dinitrogen and Carbon Monoxide. J. Am. Chem. Soc. 2017, 139, 15151-15159.

(240) Timms, P. L. Boron-fluorine chemistry. II. Reaction of boron monofluoride with acetylenes. J. Am. Chem. Soc. 1968, 90, 4585-4589.

(241) Timms, P. L. Chemistry of boron and silicon subhalides. Acc. Chem. Res. 1973, 6, 118-123.

(242) Krasowska, M.; Bettinger, H. F. Ring Enlargement of Three-Membered Boron Heterocycles upon Reaction with Organic п Systems: Implications for the Trapping of Borylenes. Chem. Eur. J. 2016, 22, 10661-10670.

(243) Pachaly, B.; West, R. Photochemical Generation of Triphenylsilylboranediyl $\left(\mathrm{C}_{6} \mathrm{H}_{5}\right)_{3} \mathrm{SiB}$ : from Organosilylboranes. Angew. Chem. Int. Ed. Engl. 1984, 23, 454-455.

(244) Braunschweig, H.; Fernández, I.; Frenking, G.; Radacki, K.; Seeler, F. Synthesis and Electronic Structure of a Ferroborirene. Angew. Chem. Int. Ed. 2007, 46, 5215-5218.

(245) Eisch, J. J.; Shafii, B.; Odom, J. D.; Rheingold, A. L. Bora-aromatic systems. Part 10. Aromatic stabilization of the triarylborirene ring system by tricoordinate boron and facile ring-opening with tetracoordinate boron. J. Am. Chem. Soc. 1990, 112, 1847-1853.

(246) Pues, C.; Berndt, A. 1-tert-Butylborirenes. Angew. Chem. Int. Ed. Engl. 1984, 23, 313-314.

(247) Habben, C.; Meller, A. Synthese und Eigenschaften von 1-[Bis(trimethylsilyl)amino]-borirenen.

Chem. Ber. 1984, 117, 2531-2537.

(248) Anderson, C. E.; Braunschweig, H.; Dewhurst, R. D. Borylene Transfer from Transition Metal Borylene Complexes. Organometallics 2008, 27, 6381-6389.

(249) Braunschweig, H.; Dewhurst, R. D.; Herbst, T.; Radacki, K. Reactivity of a Terminal Chromium Borylene Complex towards Olefins: Insertion of a Borylene into a C-H Bond. Angew. Chem. Int. Ed. 2008, 47, 59785980.

(250) Braunschweig, H.; Herbst, T.; Rais, D.; Seeler, F. Synthesis of Borirenes by Photochemical Borylene Transfer from $\left[(\mathrm{OC})_{5} \mathrm{M}=\mathrm{BN}\left(\mathrm{SiMe}_{3}\right)_{2}\right](\mathrm{M}=\mathrm{Cr}, \mathrm{Mo})$ to Alkynes. Angew. Chem. Int. Ed. 2005, 44, 7461-7463.

(251) Saillard, J. Y.; Hoffmann, R. Carbon-hydrogen and hydrogen-hydrogen activation in transition metal complexes and on surfaces. J. Am. Chem. Soc. 1984, 106, 2006-2026.

(252) Jones, W. D.; Feher, F. J. Mechanism of arene carbon-hydrogen bond activation by $\left(\mathrm{C}_{5} \mathrm{Me}_{5}\right) \mathrm{Rh}\left(\mathrm{PMe}_{3}\right)(\mathrm{H}) \mathrm{Ph}$. Evidence for arene precoordination. J. Am. Chem. Soc. 1982, 104, 4240-4242.

(253) Grigsby, W. J.; Power, P. P. Isolation and Reduction of Sterically Encumbered Arylboron Dihalides: Novel Boranediyl Insertion into C-C $\sigma$-Bonds. J. Am. Chem. Soc. 1996, 118, 7981-7988.

(254) Meller, A.; Seebold, U.; Maringgele, W.; Noltemeyer, M.; Sheldrick, G. M. Synthesis and structure of novel polycyclic species from toluene and $\mathrm{m}$-xylene and the dehalogenation product of difluoro(diisopropylamino)borane. J. Am. Chem. Soc. 1989, 111, 8299-8300.

(255) Meller, A.; Bromm, D.; Maringgele, W.; Böhler, D.; Elter, G. Zur Borylierung von Benzol und Naphthalin durch Dehalogenierungsprodukte von Dichlor(diisopropylamino)boran. J, Organomet. Chem. 1988, 347, 11-16.

(256) Ito, M.; Tokitoh, N.; Kawashima, T.; Okazaki, R. Formation of a borylene by photolysis of an overcrowded bis(methylseleno)borane. Tett. Lett. 1999, 40, 5557-5560.

(257) Bettinger, H. F. Phenylborylene: Direct Spectroscopic Characterization in Inert Gas Matrices. J. Am. Chem. Soc. 2006, 128, 2534-2535.

(258) Wang, Y.; Robinson, G. H. Carbene Stabilization of Highly Reactive Main-Group Molecules. Inorg. Chem. 2011, 50, 12326-12337.

(259) Bissinger, P.; Braunschweig, H.; Damme, A.; Dewhurst, R. D.; Kupfer, T.; Radacki, K.; Wagner, K. Generation of a Carbene-Stabilized Bora-borylene and its Insertion into a C-H Bond. J. Am. Chem. Soc. 2011, 133, 19044-19047. 
(260) Arrowsmith, M.; Böhnke, J.; Braunschweig, H.; Gao, H.; Légaré, M.-A.; Paprocki, V.; Seufert, J. Synthesis and Reduction of Sterically Encumbered Mesoionic Carbene-Stabilized Aryldihaloboranes. Chem. Eur. J. 2017, 23, 12210-12217.

(261) Bissinger, P.; Braunschweig, H.; Kraft, K.; Kupfer, T. Trapping the Elusive Parent Borylene. Angew. Chem. Int. Ed. 2011, 50, 4704-4707.

(262) Curran, D. P.; Boussonnière, A.; Geib, S. J.; Lacôte, E. The Parent Borylene: Betwixt and Between. Angew. Chem. Int. Ed. 2012, 51, 1602-1605.

(263) Braunschweig, H.; Claes, C.; Damme, A.; Deißenberger, A.; Dewhurst, R. D.; Hörl, C.; Kramer, T. A facile and selective route to remarkably inert monocyclic NHC-stabilized boriranes. Chem. Commun. 2015, 51, 1627-1630.

(264) Dahcheh, F.; Martin, D.; Stephan, D. W.; Bertrand, G. Synthesis and Reactivity of a CAACAminoborylene Adduct: A Hetero-Allene or an Organoboron Isoelectronic with Singlet Carbenes. Angew. Chem. Int. Ed. 2014, 53, 13159-13163.

(265) Ledet, A. D.; Hudnall, T. W. Reduction of a diamidocarbene-supported borenium cation: isolation of a neutral boryl-substituted radical and a carbene-stabilized aminoborylene. Dalton. Trans. 2016, 45, 9820-9826.

(266) Hudnall, T. W.; Bielawski, C. W. An N,N'-Diamidocarbene: Studies in C-H Insertion, Reversible Carbonylation, and Transition-Metal Coordination Chemistry. J. Am. Chem. Soc. 2009, 131, 16039-16041.

(267) Wang, H.; Wu, L.; Lin, Z.; Xie, Z. Synthesis, Structure and Reactivity of a Borylene Cation $\left[(\mathrm{NHSi})_{2} \mathrm{~B}(\mathrm{CO})\right]^{+}$Stabilized by Three Neutral Ligands. J. Am. Chem. Soc. 2017, 139, 13680-13683.

(268) Wang, H.; Wu, L.; Lin, Z.; Xie, Z. Transition-Metal-Like Behavior of Monovalent Boron Compounds: Reduction, Migration, and Complete Cleavage of CO at a Boron Center. Angew. Chem. Int. Ed. 2018, 57, 8708-8713. (269) Bissinger, P.; Braunschweig, H.; Damme, A.; Krummenacher, I.; Phukan, A. K.; Radacki, K.;

Sugawara, S. Isolation of a Neutral Boron-Containing Radical Stabilized by a Cyclic (Alkyl)(Amino)Carbene. Angew. Chem. Int. Ed. 2014, 53, 7360-7363.

(270) Braunschweig, H.; Dewhurst, R. D.; Hupp, F.; Nutz, M.; Radacki, K.; Tate, C. W.; Vargas, A.; Ye, Q. Multiple complexation of $\mathrm{CO}$ and related ligands to a main-group element. Nature 2015, 522, 327.

(271) Braunschweig, H.; Ye, Q.; Radacki, K. High yield synthesis of a neutral and carbonyl-rich terminal arylborylene complex. Chem. Commun. 2012, 48, 2701-2703.

(272) Légaré, M.-A.; Bélanger-Chabot, G.; Dewhurst, R. D.; Welz, E.; Krummenacher, I.; Engels, B.; Braunschweig, H. Nitrogen fixation and reduction at boron. Science 2018, 359, 896-900.

(273) Burford, R. J.; Fryzuk, M. D. Examining the relationship between coordination mode and reactivity of dinitrogen. Nat. Rev. Chem. 2017, 1, 0026.

(274) MacKay, B. A.; Fryzuk, M. D. Dinitrogen Coordination Chemistry: On the Biomimetic Borderlands. Chem. Rev. 2004, 104, 385-402.

(275) Smith, J. M.; Lachicotte, R. J.; Pittard, K. A.; Cundari, T. R.; Lukat-Rodgers, G.; Rodgers, K. R.; Holland, P. L. Stepwise Reduction of Dinitrogen Bond Order by a Low-Coordinate Iron Complex. J. Am. Chem. Soc. 2001, 123, 9222-9223.

TOC:

Metallomimetic Small Molecule Activation

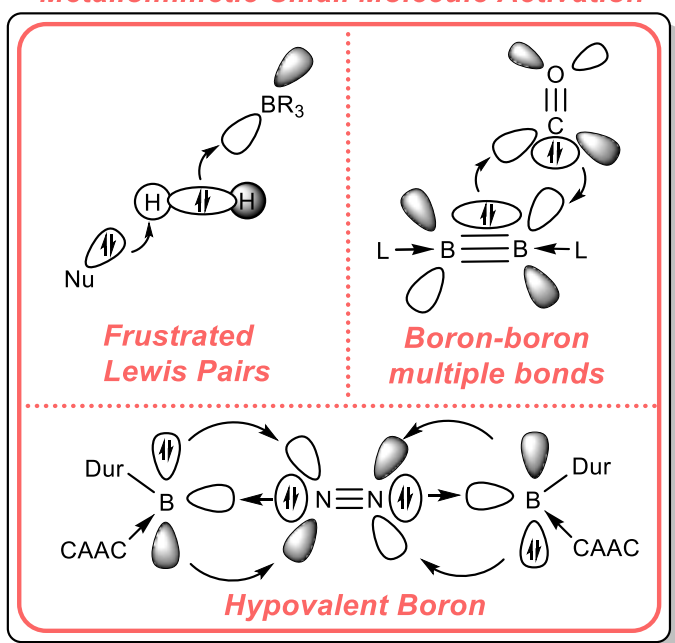

